$$
\text { DOE/ER/40492--T2 }
$$

\title{
U.C. DAVIS HIGH ENERGY PARTICLE PHYSICS RESEARCH TECHNICAL PROGRESS REPORT - 1991
}

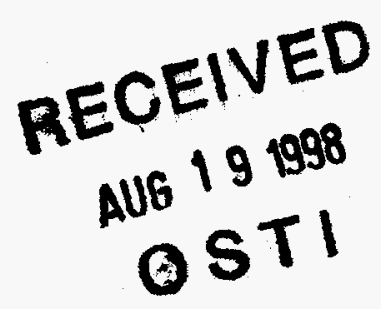

DISTRIBUTION OF THIS DOCUMENT IS UNLMITED $\$$ MASTER 


\section{DISCLAIMER}

Portions of this document may be illegible electronic image products. Images are produced from the best available original document. 


\section{DISCLAMER}

This report was prepared as an account of work sponsored by an agency of the United States Government. Neither the United States Government nor any agency thereof, nor any of their employees, makes any warranty, express or implied, or assumes any legal liability or responsibility for the accuracy, completeness, or usefulness of any information, apparatus, product, or process disclosed, or represents that its use would not infringe privately owned rights. Reference herein to any specific commercial product, process, or service by trade name, trademark, manufacturer, or otherwise does not necessarily constitute or imply its endorsement, recommendation, or favoring by the United States Government or any agency thereof The views and opinions of authors expressed herein do not necessarily state or reflect those of the United States Government or any agency thereof. 
Contents:

Task A - Experiment, $\mathrm{H} 1$ Detector at DESY

Task C - Experiment, AMY Detector at KEK

Task D - Experiment, Fixed Target Detectors at Fermilab

Task F - Experiment, PEP Detector at SLAC and Pixel Detector

Task B - Theory, Particle Physics

Task E - Theory, Particle Physics

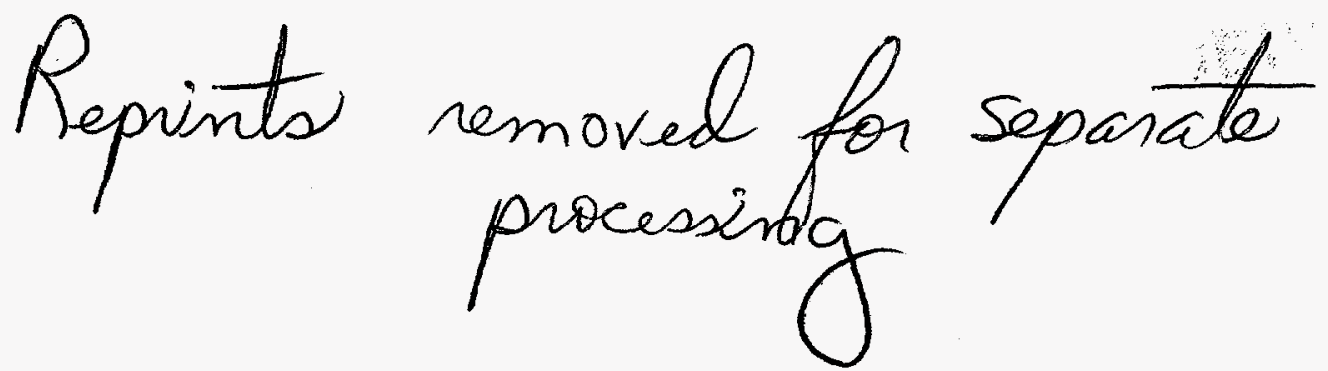




\title{
EXPERIMENTAL TASKS
}

\author{
Task A - Experiment, H1 Detector at DESY \\ Task C - Experiment, AMY Detector at KEK \\ Task D - Experiment, Fixed Target Detectors at Fermilab \\ Task F - Experiment, PEP Detector at SLAC and Pixel Detector
}




\section{TASK A}

\section{Tailcatcher.}

The return yoke for the $\mathrm{H} 1$ magnet is instrumented with planes of streamer tubes interspersed within the layers of the return yoke iron. The purpose is to measure the "tail" of the hadronic shower if it is not fully contained within the liquid argon calorimeter: hence the name "tail catcher". UCD is building the analog readout for this tailcatcher calorimeter. The construction and testing of the analog readout is proceeding on schedule.

The superboard contains line teceiver, shaper, sample-and-hold, and control cards. This densely packed and rather complicated six-layer board was designed on UCD's Mac II CAD/CAM computer. Each board can handle 128 channels of analog input signal, so a total of about forty boards including spares will be required for the full H1 detector. Our CAD tape was sent to a local vendor who supplied us with three boards; a second-source vendor supplied two additional boards. The first vendor's boards were found to be satisfactory. These were completed by adding other components at UCD and were then taken to CERN for testing in the full readout system set up by the H1 LAr calorimeter people in a CERN test beam. The readout system is rather corriplex, and we had to rely on the LAr people to operate their system for reading out these analog signals from the tailcatcher. They had their own interests, and the coordination was less than perfect. In the end, however, we managed to test the system, find its faults, and fix them.

In the process, we had to build a pulser fanout system. The calibration of the analog readout system uses test pulses from highly accurate pulser developed by French groups for LAr calibration, which has to be very accurate and stable in order to achieve the desited $1 \%$ overall tesolution in the LAt calorimeter. The system is rather expensive, however, and could not easily be expanded to accommodate the 4000 or so tailcatcher towers. Moreover, since the tailcathcher typically sees only a small fraction of the total energy of a hadronic shower, it can afford to be much less accurate - a level of $5 \%$ should suffice. Therefore, we designed a set of 64-fold fanouts to provide enough signals for all of the tailcatcher towers. The first five units have been built and tested. The 320 individual outputs deviate from the overall mean value by about $0.3 \%$. sigma. (See Figure 1).

By December 1989 we had a tested design and could begin fabrication of the full set of analog readout boards. Assembly and testing at UCD was carried out during the first half of 1990 . We now have all of the boards completed and under 
test at UCD. About 20 have been through all tests. Testing of the remainder should be finished in mid-August. Power supplies are also finished now and are being debugged. We plan to take the equipment to DESY in September and install it in the $\mathrm{H} 1$ detector. 


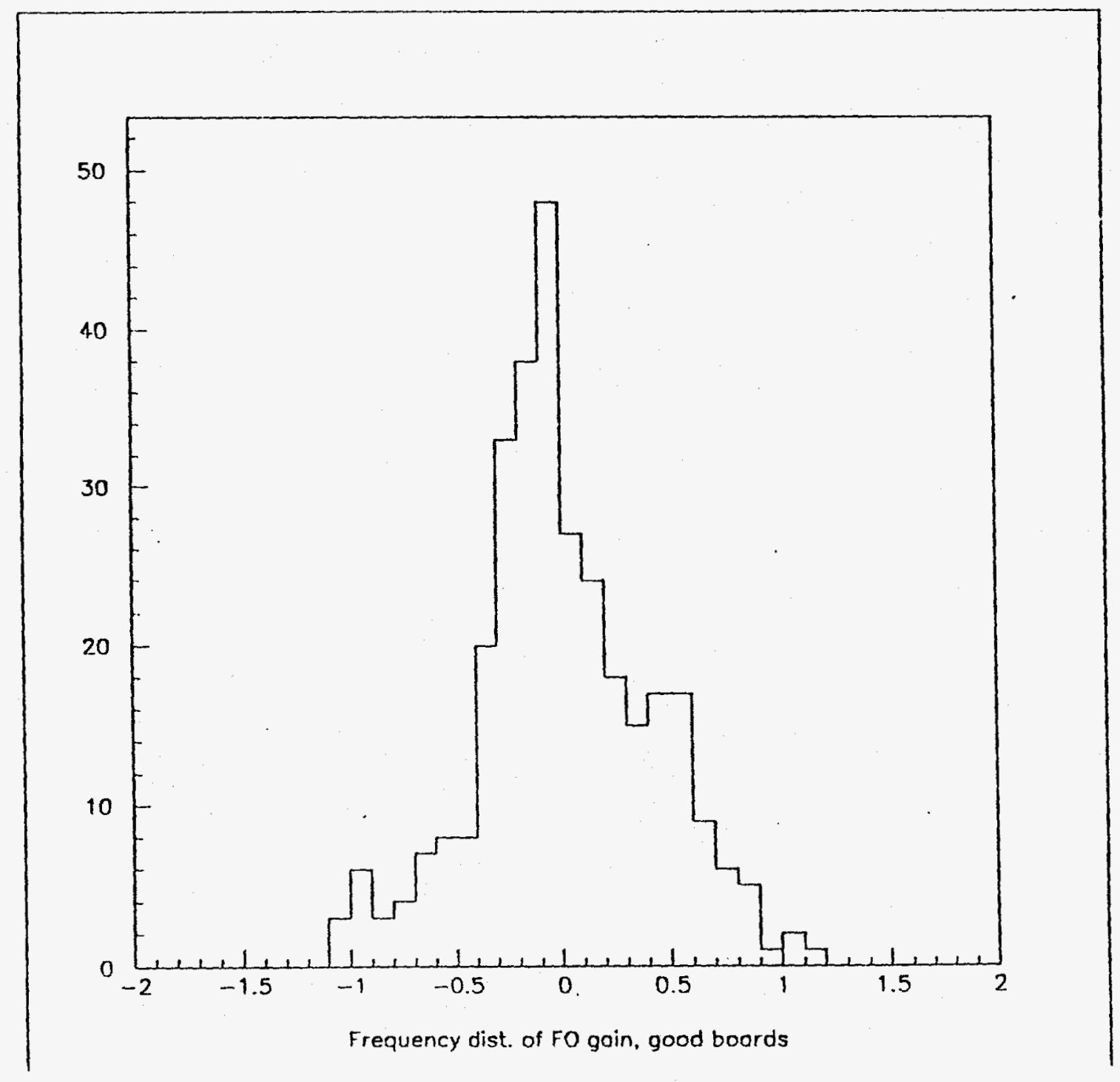

Figure 1

Percentage deviation of individual fanout channels from the mean value of a 17320 channels of the 5 fanout units. The sigma of the distribution is $0.3 \%$ 


\section{TASK C}

\section{Phrsics Impact.}

The AMY detector rolled in on November 1986, exactly two years after its formal proposal (TRISTAN-EXP-03, NOV. 1984). Physics data taking started in January 1987. Only $\$ 4.3 \mathrm{M}$ from the U.S. has been spent on the whole detector (together with $1 \mathrm{~B}$ Yen from Japan). AMY is the last TRISTAN experiment to get approved, yet initially it was one of the only two experiments taking data and the first paper was published after one year of data taking. This is the "AMY miracle."

During the last year AMY has really started reaping its fruit. Twelve papers have been published. They can be categorized as follows:

1. Search for new particles and substructures:

"Search for the Substructure of Leptons in High Energy QED Processes at TRISTAN", Phys. Lett. B223:476.

"Search for Unstable Heavy Neatral Leptons in $e^{+} e^{-}$Annihilations at $\sqrt{s}$ from

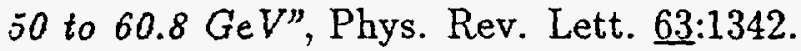

"Search for a Fourth-Generation Charge-1/3 Quark", Phys. Rev. Lett. 63:1910.

"Search for Non-Minimal Higgs Production in $e^{+} e^{-}$Annihilations at $\sqrt{s} \simeq$ $56 \mathrm{GeV}^{\prime \prime}$, Phys. Lett. B228:548.

"A Search for SUSY Particles in $e^{+} e^{-}$Annihilations at $\sqrt{s}=50-60.8 \mathrm{GeV}$ ", Phys. Lett. B234:534.

"A Search for Leptoquark and Colored Lepton Pair Production in $e^{+} e^{-}$Annihilations at TRISTAN“" Phys. Lett. B240:243. 
"Mass Limits of Charged Higgs at Large tan ( $\beta$ ) from $e^{+} \epsilon^{-}$Annihilations at $\sqrt{s}=50-60.8$ GeV", Phys. Rev. D42:949.

2. Study of forward-backward asymmetry due to electro-weak interference. TRISTAN is at the energy where this interference is maximal:

"Measurement of $e^{+} e^{-} \rightarrow b \bar{b}$ Forward-Backward Charge Asymmetry between $\sqrt{s}=52$ and $57 \mathrm{GeV}$, Phys. Rev. Lett. $\underline{63: 2341 .}$

"Forward-Backward Charge Asymmetry in $e^{+} e^{-} \rightarrow$ Hadron Jets", Phys. Rev. Lett. 64:983.

3. Study of QCD, jets and multi-hadron event properties:

"Comparison of Quark and Gluon Jets Produced in High-Energy $e^{+} e^{-}$Annihilations", Phys. Rev. Lett. $\underline{63}: 1772$.

"Multihadron-event Properties in $e^{+} e^{-}$Annihilation at $\sqrt{s}=52-57 \mathrm{GeV}$ ", Phys. Rev. D41:2675.

"Charged Hadron Multiplicities in $e^{+} e^{-}$Annihilations at $\sqrt{s}=50-61.4 \mathrm{GeV}$, Phys. Rev. D42:737.

\section{U.C. Davis Contributions to AMY Hardware and Analvsis.}

The U.C. Davis group played a crucial role in the construction of the electromagnetic shower detector by making its cathode-readout boards and read-out boards for the Endcap calorimeter in the AMY upgrade. UC Davis also built, installed, and operates the Inner Tracking Chamber (ITCh), made of "straw" tubes, and built its trigger, and the straw-type vertex chamber in the AMY upgrade. .

The construction of the "plotted". circuit boards for the electromagnetic shower detector involved the drawing and etching of over 200, S' $\times 4^{\prime}$ double sided boards. Each layer was adjusted so that the pads lined up in exact tower geometry - to do 
this, 80 different patterns had to be drawn. The purpose of exact tower geometry is to provide best possible angular resolution and angular separation between showers with a finite number of electronic readout channels. The method of fabrication of the "plotted" circuit boards is that of drawing with acid resistant ink on a large board. It is unique in the world. This ability to produce boards of such high quality is now again applied to the AMY upgrade.

To improve the hermicity of the detector, we upgraded the endcap region. The new endcap calorimeter makes use of gas-filled plastic tubes. Pickup pads on the surfaces provide readout signals in addition to those from the wires running the length of the tubes. UCD fabricated the large PC boards that held the pickup pads for the shower counterand will do the same for the endcap calorimeter. "Printing" of the designs onto the PC boards as well as the etching would be done at UCD.

The inner tracking chamber is a high precision drift chamber utilizing minimum material. It consists of polycarbonate tubes arranged to form a four-layer closedpacked array of drift cells. Each layer consists of 144 tubes. Tube technology was chosen for its cylindrically symmetric cathode structure as well as its strength. This device is the least accessible of the entire AMY detector. It must be insensitive to wire breakage. Individual tubes provide isolation between adjacent sense wires, thereby eliminating the possibility of losing the use of large segments of the chamber due to one broken wire.

An important function of the inner tracking chamber is to provide a charge track trigger. This trigger processor was designed and built by UCD. The trigger processor counts different types of track segments in the ITCh, the total number of hits, and assigns a topology number to each event. The trigger decision is made using this information. Track segments are identified by analysing the hits in a twelve tube cluster. The hits in a cluster are used to address a fast memory (4096 by 4 bits). The four outputs are used to signify different track types. The important part of this sophisticated trigger is its ease of programmability for the various track patterns and associated parameters, its continuous monitoring ability, 
and its ability to simulate the trigger by Monte Carlo events.

We (UCD and KEK) are currently constructing the new vertex chamber for the AMY detector to be installed just outside of the new smaller diameter beam pipe. The outer radius of the current AMY beam pipe is $12.5 \mathrm{~cm}$, thickness of $2 \mathrm{~mm}$ aluminum. The new beam pipe wil be $5 \mathrm{~cm}$ in radius, $1 \mathrm{~mm}$ beryllium material.

It is extremely useful to be able to determine the vertex region accurately, and for that we need high accuracy position measurements. We are aiming to produce a chamber with $r-\phi$ resolution of less than $50 \mu \mathrm{m}$, and z-resolution of approximately $1 \%$. The chamber will be a straw chamber type, and $65 \mathrm{~cm}$ long. The inner tube starts $5.2 \mathrm{~cm}$ from the beam center line. It is a 4-layer chamber similar to the ITC (Inner Tracking Chamber, already in existence at AMY).

The primary responsibility of U.C. Davis for this chamber is to select the straws and to design the front end electronics (from the chamber wire to just in front of $A D C / T D C)$. For the z-read-out, we are going to use the charge division method. To obtain better than $50 \mu \mathrm{m}$ resolution in $r-\phi$, we need to pressurize the chamber gas to more than 2 atm for the chamber gas commonly used. To fit all the electronics in near the end of the chamber, in the gas can, the electronics design has to be not only optimized for the best performance, but also very small. Actually, to have the most front end of the electronics as close as possible is essential for the good performance (high resolution), because we need to keep the signal travelling distance very short. in order to prevent the degrading of the signal and to minimize the pick up noise before getting to the preamps. Out from the chamber, the signal goes to the preamp cards, then into the postamp module. There, the signal from both ends of the wire is shaped and fed into $\mathrm{ADC}$ for the $\mathrm{Z}$ measurement, and also, separately, both ends of the signal will be summed over linearly, then discriminated and fed into the TDC module for $r-\phi$ measurement. The vertex chamber will be installed during the next major shut-down, before the end of the year.

While the majority of the AMY analysis is still carried out on site at KEK on the FACOM machine, a significant amount has been shifted to the United States. 
U.C. Davis is taking an important role in developing the U.S. analysis capability, due to both its strength in analysis and its geographic location.

The U.C. Davis group was quite successful in building its VAX-CPU power based on a cluster of three MicroVax 3200's. The analysis being done primarily at U.C. Davis now includes the jet charge F/B asymmetry and Higgs search in the tau decay mode. A data distribution procedure was established. For the U.S. collaborators to contribute significantly to physics analysis it is very important for them to obtain the data as fast as possible. We now routinely transmit some selected events through the trans-Pacific link to U.C. Davs, which in turn distributes them to other U.S. institutions. This proves to be very useful for people working on a special analysis topic to make feedback to the running of the experiment. We have a University of California 56K baud link between LBL and U.C. Davis. With the anticipated upgrade of the trans-Pacific link to $56 \mathrm{~K}$ baud in the Summer of 1989, a significant fraction of the data can be distributed in the U.S. within a day after they are taken. U.C. Davis is designated by the collaboration as the data center in the U.S. Even with the fast trans-Pacific link, not all data, especially the Monte Carlo simulated data, can be shipped through computer links. The dual EXABYTE tape units on the cluster is a very important work horse. The medium, an $8 \mathrm{~mm}$ videa cassette cartridge can pack 10 tapes worth of data (2.3 giga-byte). Thus it solved our old problem of delay caused by international shipping. There are so much data packed on the tape, that we often have to use it directly instead of off-loading onto a disk. More EXABYTE units can definitely improve the productivity of the system.

\section{TRISTAN Luminosity.}

TRISTAN is currently running at 400 inverse nanobarns per day. Major installations are scheduled this fall to greatly improve the luminosity. The improvement of the superconducting R.F. will enable the machine to carry more current and therefore double the luminosity. The installation of the micro-beta quad QCS will increase the luminosity by another factor of 2 . Starting at the beginning of 1991 , 
a luminosity of 1500 inverse nanobarns per day is expected.

\section{Phvsics Prospects}

Two of the three areas we mentioned above ( 2 and 3 ) will continue to produce interesting physics with higher luminosity, even with the start of physics with LEP. The future physics, with the improvement of luminosity, will include:

(1) Detailed study of the electroweak interference. At TRISTAN energies the electroweak interference is the largest. Detailed study of the interference effect can give a preview of physics at energies far beyond the current energy. For example, the effect of $\mathrm{Z}$ (mass $\sim 90 \mathrm{GeV}$ ) was observed in the forward/backward asymmetry of the leptons at $\operatorname{SPEAR}(\sqrt{s}<\mathrm{GeV})$. By analogy, at TRISTAN one can search for a second $Z$ with mass far above those attainable by $e^{+} e^{-}$collider of the near future. In addition to forward/backward asymmetry of the leptons, we have developed a technique to study this asymmetry in the quark sector. Figure 1 shows that our energy region is uniquely suited for such study. Furthermore, with the installation of the endcap the increase of solid angle gives us a factor of two more events for the interference study.

(2) QCD and jet studies. Our energy region is situated halfway between the PEP/PETRA energies, where such study first started, and the LEP energy, where lots of data is expected. It is an important energy region for studies to pinpoint the energy dependence of the QCD and fragmentation models.

(3) Tau and b physics. With the installation of the vertex chamber, we can use the decay vertex to tag tau and b productions. In our energy region, there is some unique physics for tau and the b. The polarization of tau due to weak effect can be observed. This is an alternative to forward/backward asymmetry for interference studies. The $\mathrm{F} / \mathrm{B}$ asymmetry of $b \bar{b}$ production is very large ( $\sim 60 \%$ in TRISTAN energies). This large asymmetry can be exploited, e.g., as a tool for the study of $b-\bar{b}$ mixing. 
(4) Two-photon physics. At TRISTAN energies, since the annihilation cross section hit the bottom, we have the minimum annihilation background for two-photon physics! Compared with the PEP/PETRA energies, the visible energy of the annihilation events moves sufficiently out so as to leave a large amount of clean two-photon events. This is particularly valuable in studying the jets produced by the two-photon process. 


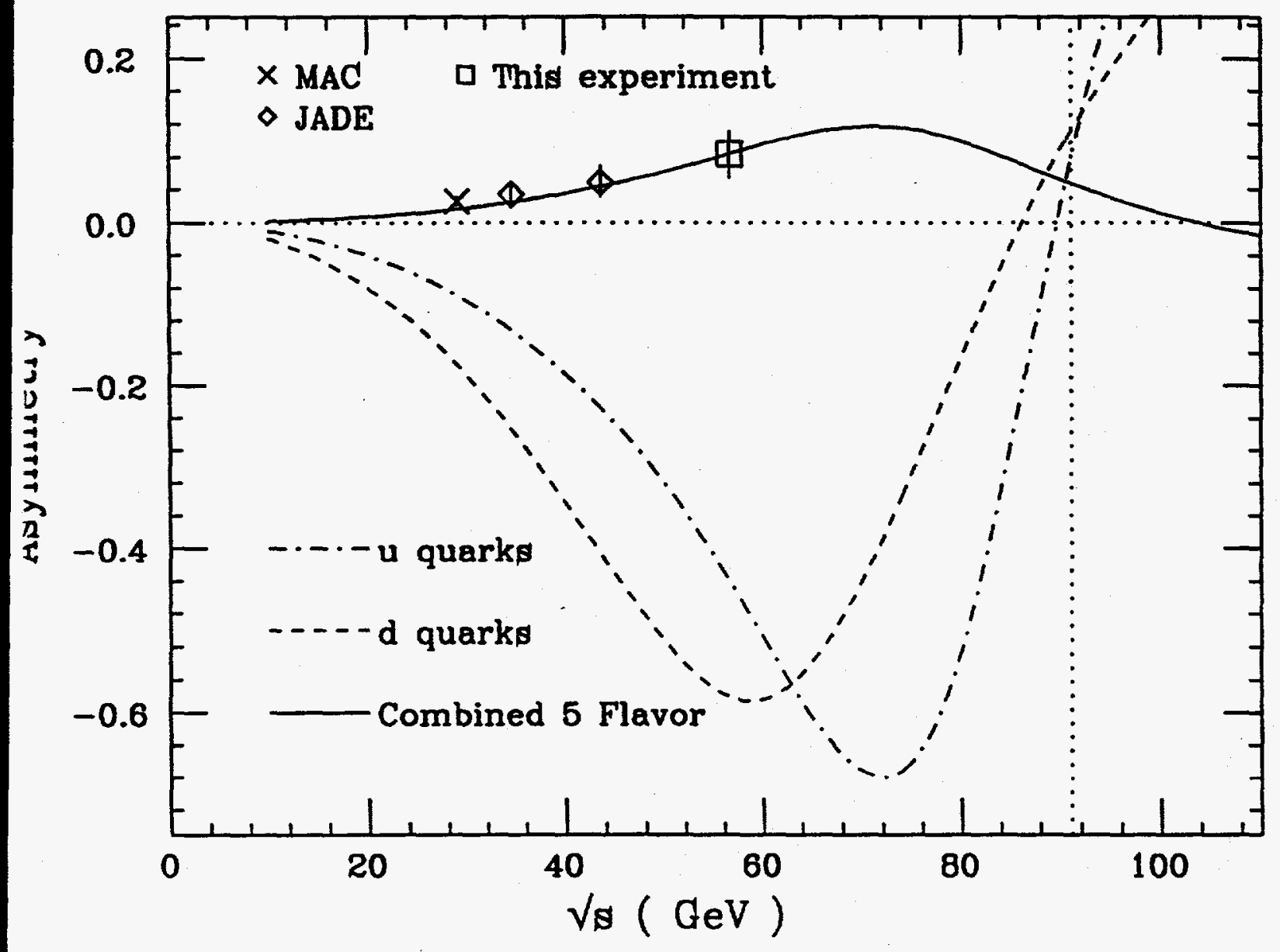

Figure 1 
Status of E653.

The first run electronic data analysis, which must precede the visual scanning of the emulsion by our colleagues in Japan, was completed in the Fall of 1987. The innovative scanning methods of the emulsion groups were new to this experiment and required a certain amount of refinement and shake-down. The success rate on finding the vertices in the emulsion on the basis of the electronic analysis is $95 \%$, far more successful than has ever been achieved with these hybrid techniques. The experimental equipment and techniques have been described in an article that appeared in April 1990. See attachment A .

As of July 1990, we have a sample of at least 200 pairs of hadronically (900 Gev proton on emulsion) produced $\mathrm{D}$ meson pairs. Two papers are currently in preparation describing the characteristics of both single particle production characteristics and pair production. A convincing and careful Monte-Carlo simulation is essential for . understanding $x_{f}$ and $p_{t}$ distributions. J. Wilcox has just completed this extensive project. A paper on the $D^{0} \rightarrow K \mu \nu / D^{0} \rightarrow \mu X$ branching ratio has been prepared and will be submitted for publication in early August. See attachment B.

The high resolution and visual capability of the emulsion will also allow us to see any unusual or unexpected short-lived phenomena that would be obliterated in most all-electronic experiments, where even observation of both members of the pair is rare. One also has the potential to directly measure the $D_{s} \rightarrow \tau$ branching ratio by careful scanning of the "kink" type charm candidates that initially appear to be $D \rightarrow \mu$, but through our careful measurement it turns out to be $D \rightarrow \tau \rightarrow \mu$, with a barely perceptible kink on the " $\mathrm{D}$ " track.

The 1987 run, as noted above, was remarkably successful. The beam was changed to deliver $600 \mathrm{GeV}$ pions, since $\mathrm{B}$ production calculations indicate that their cross section would increase at least a factor of 3 over that for $900 \mathrm{GeV}$ protons due primarily to the anti-quark content of the $\pi$. The electronic data analysis for the second run is proceeding smoothly. Extensive Monte Carlo work has been done to find methods of selection enhancing the probability of finding the B-pairs. At this time, the scanning results from the first run are almost complete, and the data reduction part through the second run is $100 \%$ complete. Predictions have been sent to the emulsion groups in Japan and they are scanning a subset with a high $p_{t} \mu$, in an effort to collect a sample of hadronically produced B particles. Very recently this search has found three very likely candidates for B pair production. Two of these events are in attachment $C$. 
UC Davis, in participating in E653, always intended to follow the hybrid experimental program with all-electronic runining in the same detector. When this was no longer feasable; other avenues were explored. The two experimental programs most seriously investigated wete P791 in the Tagged Photon Lab and E687 in the Wide Band Photon beam ( $\mathrm{p}$-West). We have joined the latter experiment. The UC Davis Micro Vax installed at the expetiment in the Fall of 1988. Additional disk and EXABYTE 8mm cartridge tape drives were added and the system was upgraded to VMS 5.1. This computer is serving at the boot-node for the local VAX cluster for E687 and has been in extensive tuse since Spting of 1989. Upon graduation, V. Paolone resumed his position as a research physicist with Task D. The inevitable departure of personinel from $\mathrm{E} 687$ left installation, repair and commisioning, as well as day to day tesponsibility for the PWC system uncovered. His experience with the large drift chambers athd electronics in $\mathrm{E} 653$ was an excellent match. Apart from the PWC's, genteral participation afid data analysis, UCD expects to develop and tefine a transverse energy trigget. Monte-Carlo work on this trigger was begut in Breckentidge, 1989 and contintued in Snowmass, 1990. The prospects for such a trigger are promising. See attachment $\mathrm{E}$, which indicates that an $E_{t}$ trigger on hadronic as well as electromagnetic energy, even with crude tesolution, is certainly a viable tool for charm enhancerment and quite likely for beauty. Studies continuue concerning another tun after the curtent fixed target tun with as much as a factor of ten more flux in the photon beam. Commitments to E653 and teaching has prevented full participation until July 1989. Since then, we have been increasingly involved with E687. Yaget spent most of summer 1989 and fall (sabbatical) at Fermilab, preparing for the fixed target fun, which begati winter 1990. Very soon, a student will be devoted to this expetitnent, the several under consideration will be taking their genefal examination in September. Although the fixed target runting period at Fermilab is in full switing with E687 recording some 4,000 events per 18 second spill ( $40 \%$ of the triggers are hadronic events from photons, the rest electron pair events), the data reduction programs are advanced and producing significant quality conttol results. These data indicate that the spectrometer is working extremely well, In attachnent $D$, the reconstructed $z$ position of the primary interaction is shown, revealing the Beryliutin tatget and other material near the target. Also showi is the effective mass plot of $K_{S}^{0} \rightarrow \pi \pi$. 
HYBRID EMULSION SPECTROMETER FOR THE DETECTION OF HADRONICALLY PRODUCED HEAVY FLAVOR STATES

K. KODAMA and N. USHIDA

Aichi University of Education, Kariya 448, Japan

R.L. LANDER, A. MOKHTARANI *, V.S. PAOLONE, J.O. WILCOX and P.M. YAGER

University of California (Davis), Davis, CA 95616, USA

R.M. EDELSTEIN, A.P. FREYBERGER, D.B. GIBAUT, R.J. LIPTON, W.R. NICHOLS, D.M. POTTER, J.R. RUSS and Y. ZHANG

Carnegie-Mellon University, Pittsburgh, PA 15213, USA

H.I. JANG, J.Y. KIM and M.Y. PAC

Chonnam National University, Kwangju 500-757, Korea

B.R. BALLER and R.J. STEFANSKI

Fermi National Accelerator Laboratory. Batavia, IL 60510, USA

K. NAKAZAWA and S. TASAKA

Gifu University, Gifu 50I-1I, Japan

Y.S. CHOI, K.H. CHUNG, D.C. KIM, I.G. PARK, J.S. SONG and C.S. YOON

Gyeongsang Na:ional University, Jinju 660-300, Kored

\section{CHIKAWA}

Kinki Unversity, Higashi-Osaka 577, Japan

T. ABE, T. FUJII, G. FUJIOKA, K. FUJIWARA, H. FUKUSHIMA, T. HARA, Y. TAKAHASHI, K. TARUMA, Y. TSUZUKI and C. YOKOYAMA

Kobe University, Kabe 657, Japan

S.D. CHANG, B.G. CHEON, J.H. CHO, J.S. KANG, C.O. KIM, K.Y. KIM, T.Y. KIM, J.C. LEE, S.B. LEE, G.Y. LIM, I.T. LIM, S.W. NAM, T.S. SHIN, K.S. SIM and J.K. WOO

Korea University, Seoul 136-701, Korea

\section{Y. ISOKANE, Y. TSUNEOKA}

Nagoya Institute of Technology. Nagoya 466, Japan

S. AOKI, A. GAUTHIER •* , K. HOSHINO, H. KITAMURA, M. KOBAYASHI, M. MIYANISHI, K. NAKAMURA, M. NAKAMURA, Y. NAKAMURA, S. NAKANISHI, K. NIU, K. NIWA and $H$. TAJIMA

Nagova Ciniversity, Nagoya 464-01, Japan

- Present address: Fermi National Accelerator Laboratory. Batavia, IL 60510. USA.

* Present address: University of Illinois, Urbana-Champaign, IL 61801. USA. 
J.M. DUNLEA ***, S.G. FREDERIKSEN, S. KURAMATA *, B.G. LUNDBERG *, G.A. OLEYNIK *, N.W. REAY, K. REIBEL, C.J. RUSH, R.A. SIDWELL and N.R. STANTON

The Ohio State University, Columbus, OH 43210, USA

K. MORIYAMA and H. SHIBATA

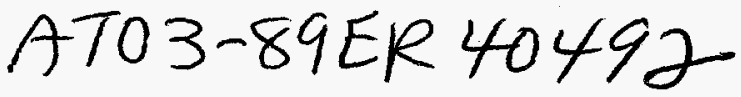

Okayama University, Okayama 700, Japan

T.S. JAFFERY * , G.R. KALBFLEISCH, P.L. SKUBIC, J.M. SNOW, S.E. WILLIS ${ }^{++}$and W.Y. YUAN

University of Oklahoma, Norman, OK 73019, USA

O. KUSUMOTO ${ }^{+++}$, T. OKUSAWA, M. TERANAKA, T. TOMINAGA, T. WATANABE and J. YAMATO

Osaka City University, Osaka 558, Japan

\section{H. OKABE and J. YOKOTA}

Science Education Institute of Osaka Prefecture, Osaka 558. Japan

M. KAZUNO, F. MINAKAWA, E. NIU, S. ONO, H. SHIBUYA and S. WATANABE

Toho University, Funabashi 274, Japan

Y. SATO and I. TEZUKA

Utsunomiya University, Utsunomiya 350. Japan

S.Y. BAHK and S.K. KIM

Wonkwang University, Iri 570-749, Korea

Received 16 October 1989

A hybrid apparatus consisting of a movable emulsion target and a magnetic spectrometer was used in a fixed target Fermilab Tevatron experiment to study the production of heavy quarks by high-energy hadron beams. High-resolution silicon microstrip detectors were used for precise tracking in the dense particle environment. Details of the experimental apparatus, including the data acquisition system, are described.

\section{Introduction}

The discovery of heavy quarks and the $T$ lepton introduced to the world particles with lifetimes between 0.1 and 1.0 ps. We describe here a detector capable of direct observation of such particles which was used in Fermilab Experiment E653. This detector was an extension of the hybrid emulsion technique developed for a previous Fermilab experiment (E531) [1] and was optimized for a hadron instead of a neutrino beam. While

- Present address: Fermi National Accelerator Laboratory, Batavia, IL 60510, USA.

*** Present address: University of Rochester, NY 14627, USA.

+ Present address: Nagoya University, Nagoya 464, Japan.

++ Present address: Northern Illinois University, DeKalb, IL 60115. USA.

${ }^{+++}$Present address: Soai University, Nanko-naka 4-4-1, Suminoe-ku. Osaka 559. Japan. in the E531 neutrino experiment charm was produced in one of every twenty charged current interactions in the emulsion target [2], only one hadronic interaction in a thousand produces charm and one in a million beauty. Thus, greater discrimination against nonheavy quark background was required in E653 to limit emulsion scanning time. To achieve this, a high-resolution electronic spectrometer was placed downstream of the emulsion. The spectrometer was capable of precisely locating events within the emulsion as well as providing information about the decay products. The apparatus was triggered by an interaction in the emulsion and a penetrating muon.

There were two experimental runs. The first (May to August 1985) used an $800 \mathrm{GeV}$ proton beam and concentrated on charm; the second (August to November 1987 ) used a $600 \mathrm{GeV}$ negative pion beam and the emphasis was on extracting a sample of B mesons from the data. Some modifications to the apparatus were 
made between the runs to facilitate the emulsion scanning and to improve the geometrical acceptance, the muon trigger, and the on-line data acquisition system.

\section{Components of the spectrometer}

Because of its unsurpassed spatial resolution, nuclear emulsion was chosen as the "active" target for this experiment. Emulsion modules several centimeters thick were mounted on a target mover which ensured uniform exposure to the beam. The electronic detectors downstream of the target made possible accurate predictions of primary and secondary vertices for the emulsion scanning, and provided momentum measurement and particle identification.

Fig. 1 shows a layout of the experimental apparatus [3]. The desired precision for vertex location required the use of silicon strip detectors (SSDs). Sets of these detectors were placed on both sides of the emulsion target; the upstream (beam) set was used to measure the beam track positions and was given a large lever arm by small drift chambers several meters upstream. Tracks from interactions and decays in the emulsion were measured in the downstream silicon microstrip detectors (vertex SSDs) which were followed by a dipole inagnet and a set of high-precision drift chambers, producing a combination capable of measuring charged particle momentum with large acceptance. In addition, the drift chambers provided a link between the upstream vertex SSDs and the downstream components of the experiment. The noise-sensitive silicon strip detectors and associated electronics together with the emulsion target mover and beam defining counters were inside an rf-shielded climate-controlled house. A timeof-flight system (TOF), lead and liquid-argon electromagnetic calorimeter (LAC), and hadron calorimeter (HadCal) provided limited particle identification and energy measurements. Only muons above $5 \mathrm{GeV}$ could penetrate the steel absorbers and traverse the downstream muon spectrometer, which consisted of drift chambers upstream and downstream of a toroid magnet followed by two walls of scintillator hodoscopes separated by steel; a coincidence signal from both walls was required by the trigger. The muon spectrometer could measure the trajectories and momenta of the muons and permitted longitudinal and transverse momentum cuts for charm and beauty selection [4].

An event trigger consisted of a beam track producing three or more charged tracks, with at least one of them satisfying the muon trigger described above. Data from the experiment.were read into a front-end LSI-11/73 computer through Fastbus and Camac data acquisition systems. After minor event assembly on this computer, events were transferred to a VAX-11/750 computer where an on-line system monitored data and equipment and wrote events to magnetic tape.

\subsection{The beam}

It was expected that the cross section for hadronic heavy flavor production at the Tevatron would depend strongly on energy and therefore the first run was done with the highest available energy, namely $800 \mathrm{GeV}$ protons. Since it is likely that pions are more effective than protons at producing beauty [5], a $600 \mathrm{GeV}$ negative pion beam was used in the second run. The experi-. ment was situated Lab D in the Neutrino Area-East

\section{E653 Elevation View}

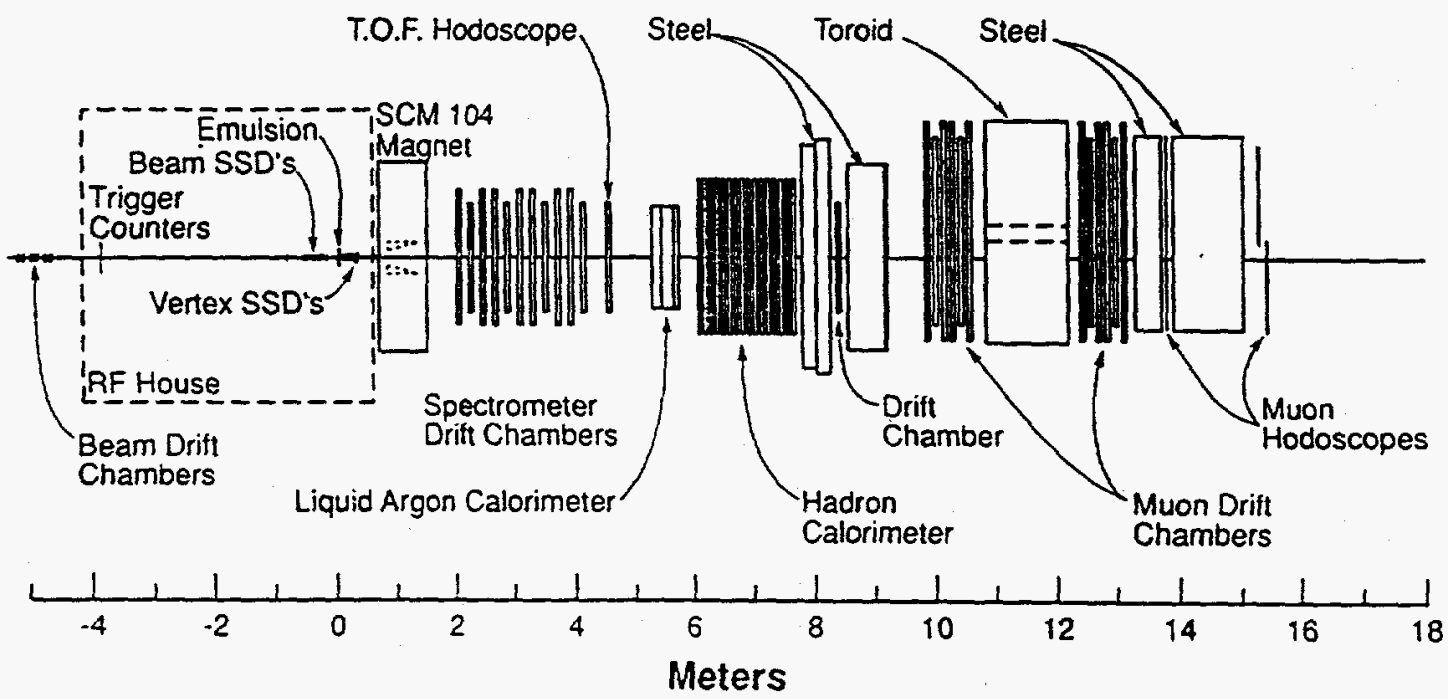

Fig. 1. Plan view of E-653 detector. 
beamline of Fermilab. Secondary beams were generated by targeting between $10^{11}$ and $10^{12}$ protons per pulse. The repetition rate was one pulse every $57 \mathrm{~s}$ in both runs, with a $10 \mathrm{~s}$ spill in the first run and a $20 \mathrm{~s}$ spill in the second.

The secondary beam was run at $2 \times 10^{5}$ particles per pulse and was limited by the rate at which the emulsion could be moved through the beam. The beam was foclised on the emulsion target in a spot with an rms size of $1 \mathrm{~mm}$ to optimize the spectrometer acceptance of secondaries. A series of scintillation counters defined the beam and vetoed the beam halo (see section 3). The halo was kept at less than $7 \%$ integrated over the area within a $10 \mathrm{~cm}$ radius around the beam to keep the emulsion from being unnecessarily exposed.

\subsection{Bean drift chambers and beam SSDs}

The beam drift chambers, located $5 \mathrm{~m}$ upstream from the target consisted of 18 planes of active area $9^{\circ}$ $\mathrm{cm}$ by $9 \mathrm{~cm}$ divided into three groups of six planes each arranged $u u^{\prime} x x^{\prime} v v^{\prime}$ with $x$ the horizontal readout direction and $u$ and $v$ referring to $60^{\circ}$ and $120^{\circ}$ rotations respectively; the primes indicate that corresponding sense wires are staggered. All of the planes were enclosed in a single vessel with mylar beam windows, which was filled with $50 \%$ argon and $50 \%$ ethane, the same gas mixture used by the spectrometer and muon drift chambers. (The gases for the three drift chamber systems differed only in the amount of alcohol doping and were closely monitored with a gas chromatograph)

The beam silicon strip detectors (beam SSDs) consisted of nine planes arranged in sets of three oriented $60^{\circ}$ apart. The strips had a pitch of $20 \mu \mathrm{m}$ with every third line read out in the central region and every sixth in the outer region. On each plane the 144 channeis nearest the beam spot were instrumented. Hybrid preamplifiers were capacitively coupled to each silicon diode strip; their outputs went to a post-amplifier for delay-line pulse shaping. Its output was de coupled into an ADC so that the pedestal level could be controlled by adjusting the dc output voltage.

The beam SSDs, the emulsion target mover, and the vertex SSDs were all mounted on stands resting on a large granite block, in order to minimize relative motion among these components. The large "lever arm" between the beam SSDs and drift chambers in conjunction with the precision of the microstrip detectors provided an rms transverse position error for beam tracks projected to the target of $10 \mu \mathrm{m}$ and an rms slope of 20 $\mu \mathrm{rad}$.

\subsection{The target emulsion system}

In the first run 49 target modules containing a total of 33 liters of Fuji-ET-7B nuclear emulsion were ex-

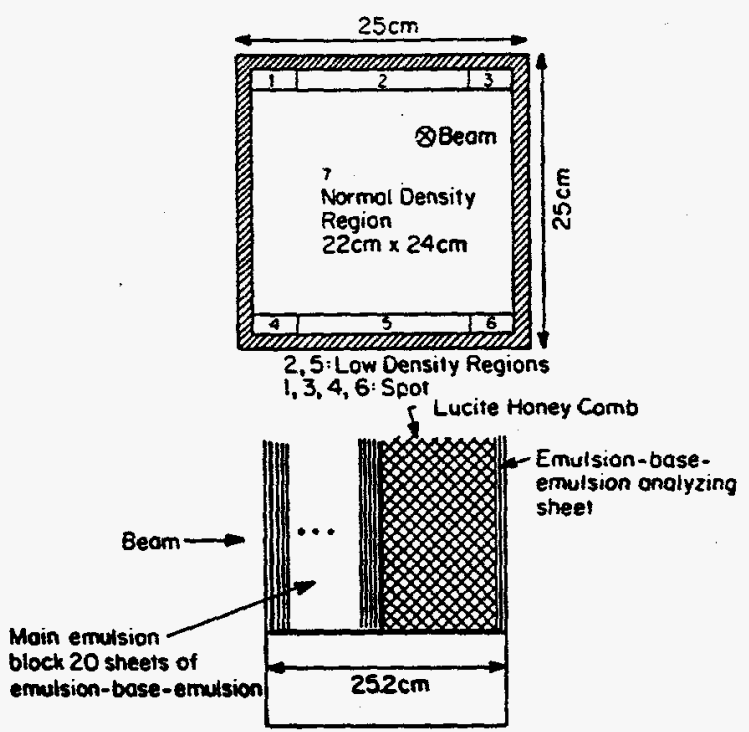

Fig. 2. Vertical emulsion module.

posed. The corresponding numbers for the second run were 53 modules totaling 38 liters. The emulsion was poured at Fermilab immediately before exposure, and developed both at Fermilab and at Nagoya University. Each emulsion target was encased in a laminated sheet and was loaded into an aluminum frame of a target mover which exposed single target modules to the beam so that the typical density of beam tracks was uniform over the exposed emulsion.

The composition of the nuclear emulsion was similar to that given in ref. [1]. Its average atomic weight, $\langle A\rangle$, was 26.6 , computed from $\langle A\rangle=1 /\left(\Sigma_{i}\left(W_{i} / A_{i}\right)\right)$, where $W_{i}$ and $A_{i}$ are the fractional weights and atomic weights respectively of the constituent atoms. Two types of target modules were employed; 24 of the first-run and 31 of the second-run modules were "vertical" and the rest "horizontal". The vertical modules had their emulsion sheet planes oriented perpendicular to the beam, while the horizontal modules were oriented parallel to the beam. In the first run, vertical modules were exposed at $1.5 \times 10^{5}$ protons $/ \mathrm{cm}^{2}$, and the horizontal at an average of $0.8 \times 10^{5}$ protons $/ \mathrm{cm}^{2}$. The second-run exposures were $3.0 \times 10^{5}$ pions $/ \mathrm{cm}^{2}$ and $1.0 \times 10^{5}$ pions $/ \mathrm{cm}^{2}$ respectively.

Fig. 2 shows the construction of the vertical module. Each module consisted of twenty thick plates and a thin plate [6]. The thick plates had an emulsion layer of 330 $\mu \mathrm{m}$ on each side of a $25 \mathrm{~cm} \times 25 \mathrm{~cm} \times 70 \mu \mathrm{m}$ polystyrene plate and formed the main target block, while the downstream thin plate had an emulsion layer of 70 $\mu \mathrm{m}$ on each side of a $25 \mathrm{~cm} \times 25 \mathrm{~cm} \times 500 \mu \mathrm{m}$ lucite plate and was separated from the main block by a 10 $\mathrm{mm}$ thick honeycomb [7] board, the latter combination being considered the analyzing region. Fig. 3 shows the 


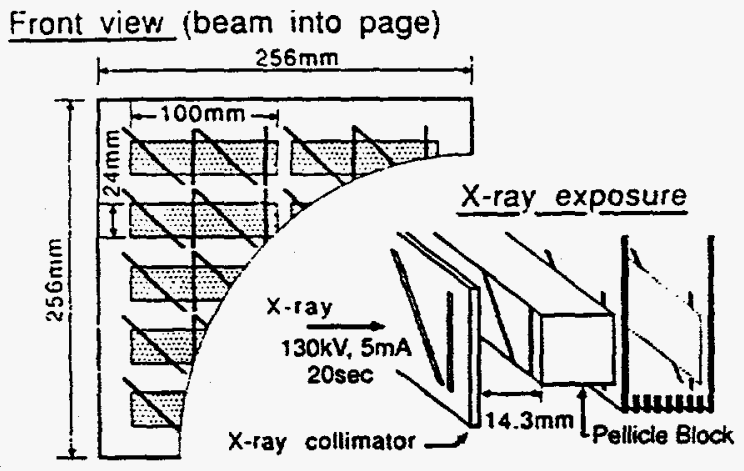

Side view (beam from left to right)

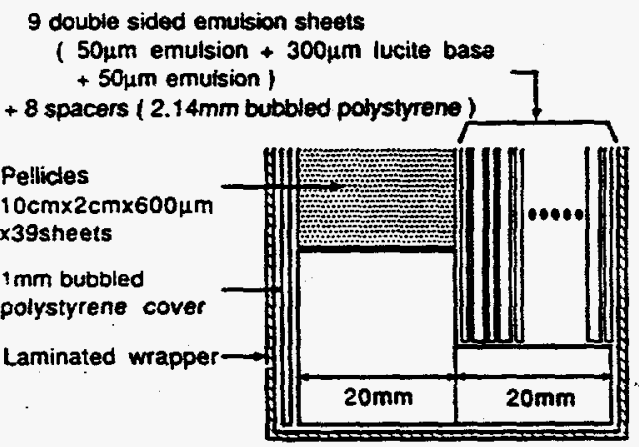

Fig. 3. Horizontal emulsion module. makeup of the horizontal target module [8]. Each of the ten mini-modules consisted typically of 39 pellicles 2 $\mathrm{cm} \times 10 \mathrm{~cm} \times 600 \mu \mathrm{m}$ for the main target block followed by an analyzing region consisting of nine vertical type thin plates having $50 \mu \mathrm{m}$ emulsion on each side of a $23.5 \mathrm{~cm} \times 23.5 \mathrm{~cm} \times 300 \mu \mathrm{m}$ lucite plate separated by $2.14 \mathrm{~mm}$ low density bubbled polystyrene plates. Both vertical and horizontal modules were wrapped in a laminated sheet of four layers containing (from the outside inwards) $40 \mu \mathrm{m}$ paper, $40 \mu \mathrm{m}$ polyethylene and polyisobutylene mixture, $10 \mu \mathrm{m}$ aluminum, and $110 \mu \mathrm{m}$ black polyethylene. Tables 1 and 2 give values of the relevant physical properties of the materials contained in each type of module.

The analyzing regions assisted in the location of the decays of long-lived particles, and were also used to improve the vertex predictions of the solid state microstrip detectors by providing precise tracking points near the main emulsion block. The analyzing regions were carefully designed to optimize beauty and charm yield with minimal losses due to multiple scattering and secondary interactions in the material.

The emulsion modules were mounted on a target mover and moved through the beam during the twenty second slow spill (ten seconds during the first run). The horizontal sweep speed of the target mover was varied using real-time beam rate information obtained from an

Table 1

Vertical emulsion target module and tape composition.

Emulsion volume per module $=0.83$ (main block) +0.01 (analyzing region) $=0.84$ liter total

\begin{tabular}{|c|c|c|c|c|c|c|}
\hline Section & Item & $\begin{array}{l}\left.\text { Length }{ }^{*}\right) \\
\text { [mm] }\end{array}$ & Number & $\begin{array}{l}\text { Total } \\
\text { length } \\
\text { [mm] }\end{array}$ & $\begin{array}{l}\text { Fractional } \\
\text { interaction } \\
\text { lengths }{ }^{b}\end{array}$ & $\begin{array}{l}\text { Fractional } \\
\text { radiation } \\
\text { lengths }\end{array}$ \\
\hline \multirow[t]{4}{*}{ Target } & Wrapper & 0.24 & 1 & 0.24 & 0.0003 & 0.0006 \\
\hline & Emulsion sheet & 0.33 & $2 \times 20$ & 13.20 & 0.037 & 0.447 \\
\hline & Polystyrene base & 0.07 & 20 & 1.40 & 0.002 & 0.003 \\
\hline & Totals (target) & & & 14.84 & 0.0393 & 0.4506 \\
\hline \multirow{5}{*}{ Analyzer } & Lucite honeycomb & 10.0 & 1 & 10.0 & 0.0012 & 0.0032 \\
\hline & Emulsion & 0.07 & $2 \times 1$ & 0.14 & 0.0004 & 0.0047 \\
\hline & Lucite base & 0.50 & 1 & 0.50 & 0.0007 & 0.0015 \\
\hline & Wrapper & 0.24 & 1 & 0.24 & 0.0003 & 0.0006 \\
\hline & Totais (analyzer) & & & 10.88 & 0.0026 & 0.0100 \\
\hline Emulsion & Emulsion & 0.060 & $2 \times 1$ & 0.120 & 0.0003 & 0.0041 \\
\hline & Triacetate base & 0.200 & 1 & 0.200 & 0.0003 & 0.0006 \\
\hline & Windows & 0.220 & $2 \times 1$ & 0.440 & 0.0005 & 0.0011 \\
\hline & Totals (tape) & & & 0.760 & 0.0011 & 0.0058 \\
\hline Toials & target + analyzer + tape & & & 26.48 & 0.043 & 0.47 \\
\hline
\end{tabular}

") Lengths are along beam direction.

b) Our measured value for $800 \mathrm{GeV}$ protons on emuision is $330 \pm 20 \mathrm{~mm}$; most other values computed from Particle Data Group table. 
Table 2

Horizontal emulsion tárget nodule composition.

Emulsion volume per module $=0.47$ (main block) +0.05 (analyzing region) $=0.52$ liter total.

\begin{tabular}{lllllll}
\hline Section & Item & $\begin{array}{l}\text { Length }{ }^{2)} \\
{[\mathrm{mm}]}\end{array}$ & Number & $\begin{array}{l}\text { Total } \\
\text { length } \\
\text { [mm] }\end{array}$ & $\begin{array}{l}\text { Fractional } \\
\text { interaction } \\
\text { lengths }\end{array}$ & $\begin{array}{l}\text { Fractional } \\
\text { radiation } \\
\text { lengths }\end{array}$ \\
\hline Target & Wrapper & 0.20 & 1 & 0.20 & 0.0002 & 0.0005 \\
& $\begin{array}{l}\text { Bubbled polystyrene } \\
\text { Emulsion pellicle }\end{array}$ & 1.00 & 1 & 1.00 & 0.0001 & 0.0002 \\
& Totals (target) & & 1 & 20.0 & 0.056 & 0.678 \\
& Emulsion & & & 21.20 & 0.0563 & 0.6787 \\
Analyzer & Lucite & 0.05 & $2 \times 9$ & 0.90 & 0.003 & 0.031 \\
& Bubbled polystyrene & 0.30 & 9 & 2.70 & 0.004 & 0.008 \\
& Wrapper & 0.14 & 8 & 17.12 & 0.002 & 0.003 \\
& Totals (analyzer) & & 1 & 0.20 & 0.0002 & 0.0005 \\
Totals & target + analyzer & & & 20.92 & 0.0092 & 0.0425 \\
\hline
\end{tabular}

1) Lengths are along beam direction.

b) Our measured value for $800 \mathrm{Gev}$ protons on emulsion is $330 \pm 20 \mathrm{~mm}$; most other values computed from Particle Data Group table.

upstream scintillation counter, so that the emulsion was exposed uniformly to the beam. At the end of each horizontal sweep, the target was moved vertically by 1 $\mathrm{mm}$ so that the exposure density along the vertical axis was nearly uniform. The movement of the target was controlled using a digital on-line' microscope system (DOMS) which is a front-end processor originally developed at Nagoya to control the microscope stage in semi-automatic emulsion analysis (see section 5). A position encoding system recorded the horizontal and vertical coordinates of the target mover with a precision of $10 \mu \mathrm{m}$ whenever a pre-trigger occurred. A full trigger

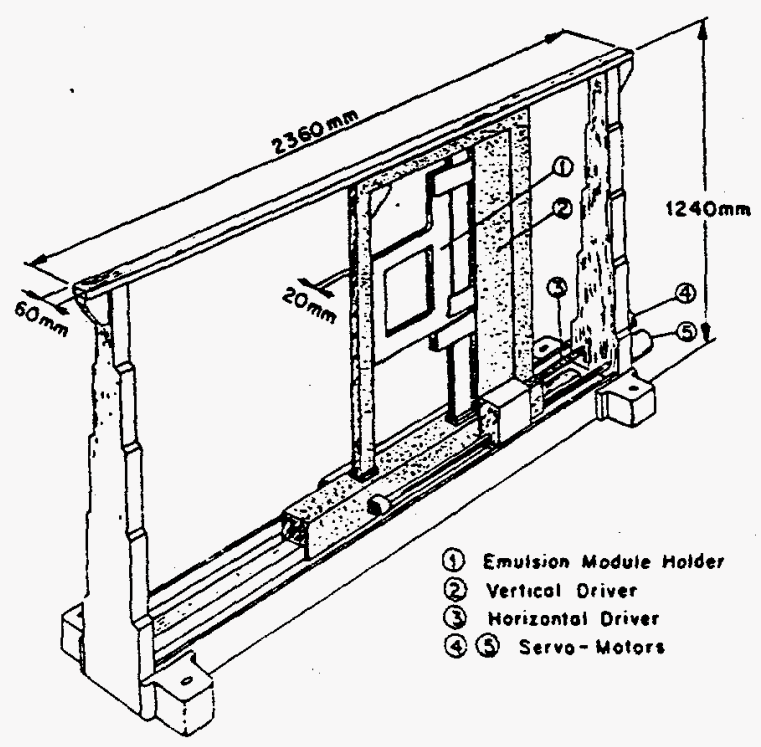

Fig. 4. Emulsion target mover. resulted in the readout of the coordinates through the Camac system. The target mover is shown in fig. 4.

For the second run, a roll of triacetate tape $150 \mathrm{~m}$ long, $35 \mathrm{~mm}$ wide and $200 \mu \mathrm{m}$ thick coated on each side with $60 \mu \mathrm{m}$ thick Fuji ET-7B emulsion over the central $16 \mathrm{~mm}$ width, was placed immediately downstream of the vertical modules. This emulsion tape was driven to keep its exposure density at less than $1.0 \times 10^{5}$ pions/ $\mathrm{cm}^{2}$ and permitted reliable quick tracking between the downstream Vertex SSDs and the main emulsion block, which was subjected to an exposure density of $3.0 \times 10^{5}$ pions $/ \mathrm{cm}^{2}$ for vertical modules. (Because of the number of charged particles produced in an interaction the actual particle density in the interesting regions was considerably higher [9].) The tape contributed $1.5 \%$ to the total interaction length and 19 to the total radiation length shown in table 1. Fig. 5 shows the position of the emuision tape and drive.

The horizontal modules were developed at a facility built at Fermilab; the vertical modules and emulsion tapes were transported to Japan and developed at Nagoya University. The developing methods for the emulsions were identical to those employed in Experiment E531 [1]. Before development the pellicles of the horizontal modules were mounted in groups of five on a $12 \mathrm{~cm} \times 12 \mathrm{~cm} \times 2 \mathrm{~mm}$ acrylic plate with their $10 \mathrm{~cm}$ sides touching so as to approximate a single $10 \mathrm{~cm} \times 10$ $\mathrm{cm}$ pellicle. The vertical modules, after processing, were each cut into sixty-four $3 \mathrm{~cm} \times 3 \mathrm{~cm}$ mini-blocks. The emulsion sheets of each mini-block were then separated and mounted in sequential order in five rows and five columns on a $20 \mathrm{~cm} \times 20 \mathrm{~cm}$ acrylic plate. For both runs, the first four rows consisted of the 20 thick target 


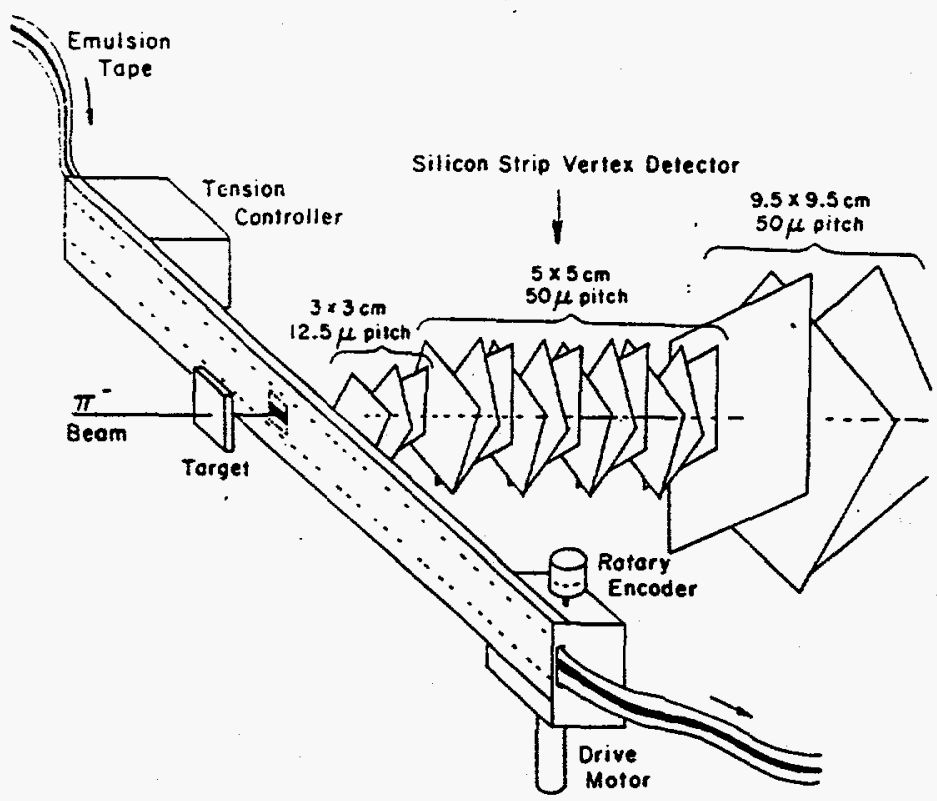

Fig. 5. Emulsion tape drive.

block plates. For the first run the fifth row contained ive analysis plates, while for the second run only one analysis plate was used. Reference marks added before development assured proper alignment of the plates after separation.

Calibration of the position of both types of modules relative to the beam and vertex silicon microstrip coun. ters was done by exposing a small portion of each module at low track density, permitting quick match-up between the spectrometer and emulsion tracks in these regions. Once a beam track was found, the module could be aligned to the spectrometer with the $10 \mu \mathrm{m}$ accuracy of the spectrometer beam track measurement. A prior, coarser, alignment of $100 \mu \mathrm{m}$ was done with the "beam spots" which were produced on the comers of the modules by keeping the target mover stationary for a certain time. The relative orientation and position of all the emulsion components in the horizontal target modules (pellicles and vertical type sheets) were. calibrated by exposing them to $X$-ray lines from a Toshiba EX-220GH X-ray machine placed upstream and to the side of the target mover [8]. Targets were moved to a preselected position out of the beam, where they were exposed to two $X$-ray lines that were angled $45^{\circ}$ with respect to one another as shown in fig. 3 . The mean linewidth of the X-ray beam was $50 \mu \mathrm{m}$. The relative alignment among all components making up the horizontal module and between the module and the spectrometer were determined to better than $20 \mu \mathrm{m}$ using these $\mathrm{X}$-ray lines.

\subsection{The spectrometer microstrip detectors}

The vertex silicon strip detectors (VSSDs) consisted of 18 plaries placed every $9 \mathrm{~mm}$ along the beam line with the first plane $47 \mathrm{~mm}$ downstream of the emulsion target. These detectors were arranged in six groups of three with the most upstream of the three oriented for horizontal readout (vertical strips) followed by $120^{\circ}$ and $60^{\circ}$ readout rotations with respect to the first. (Directions $x, v$, and $u$ ). The three most upstream planes were $3 \mathrm{~cm}$ square, followed by twelve $5 \mathrm{~cm}$ square and three $10 \mathrm{~cm}$ square [10]. (The latter were actually $9.67 \mathrm{~cm}$ wide by $9.05 \mathrm{~cm}$ high and consisted of three wafers mounted side-by-side, with the central section $2.56 \mathrm{~cm}$ wide and the outer ones $3.47 \mathrm{~cm}$ each with $0.05 \mathrm{~cm}$ dead-spaces at the junctions of the wafers.) All

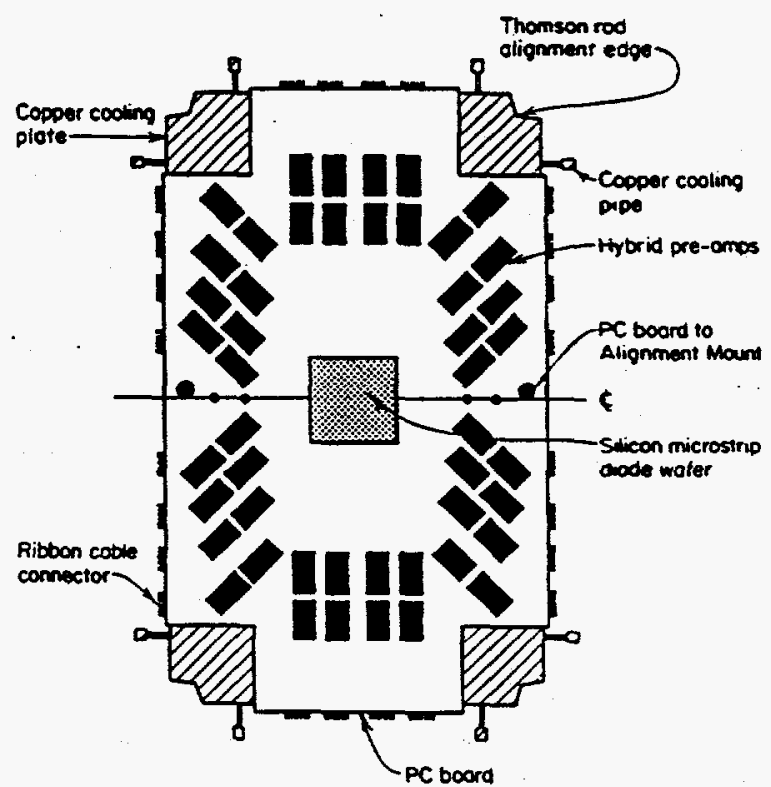

Fig. 6. Mounted VSSD plane. 
Table 3

VSSD readout spacing for the three detector sizes

\begin{tabular}{|c|c|c|c|c|c|}
\hline & $\begin{array}{l}\text { Distance } \\
\text { from center } \\
\text { [mm] }\end{array}$ & $\begin{array}{l}\text { Actual } \\
\text { strip pitch } \\
{[\mu \mathrm{m}]}\end{array}$ & $\begin{array}{l}\text { Readout } \\
\text { pitch } \\
{[\mu \mathrm{m}]}\end{array}$ & Lines & $\begin{array}{l}\text { Total lines } \\
(\text { lines } \times 2)\end{array}$ \\
\hline $3 \mathrm{~cm}$ & $\begin{array}{r}0-3.0 \\
3.0-4.1 \\
4.1-5.1 \\
5.1-6.6 \\
6.6-11.6 \\
11.6-16.6\end{array}$ & $\begin{array}{l}12.5 \\
25 \\
25 \\
30 \\
50 \\
100\end{array}$ & $\begin{array}{r}25 \\
50 \\
100 \\
150 \\
250 \\
500\end{array}$ & $\begin{array}{r}120 \\
22 \\
10 \\
10 \\
20 \\
10\end{array}$ & $\begin{array}{r}240 \\
44 \\
20 \\
20 \\
40 \\
20\end{array}$ \\
\hline \multicolumn{5}{|c|}{ Readout lines per $3 \mathrm{~cm}$ detector } & 384 \\
\hline $5 \mathrm{~cm}$ & $\begin{array}{r}0-4.0 \\
4.0-7.6 \\
7.6-10.0 \\
10.0-25.0\end{array}$ & $\begin{array}{l}50 \\
50 \\
50 \\
50\end{array}$ & $\begin{array}{r}50 \\
100 \\
150 \\
250\end{array}$ & $\begin{array}{l}80 \\
36 \\
16 \\
60\end{array}$ & $\begin{array}{r}160 \\
72 \\
32 \\
120\end{array}$ \\
\hline \multicolumn{5}{|c|}{ Readout lines per $5 \mathrm{~cm}$ detector } & 384 \\
\hline $10 \mathrm{~cm}$ & $\begin{array}{c}0-12.8 \\
13.3-48.0\end{array}$ & $\begin{array}{l}50 \\
50\end{array}$ & $\begin{array}{r}50 \\
200\end{array}$ & $\begin{array}{l}256 \\
176\end{array}$ & $\begin{array}{l}512 \\
352\end{array}$ \\
\hline \multicolumn{5}{|c|}{ Readout lines per $10 \mathrm{~cm}$ detector } & 864 \\
\hline
\end{tabular}

detectors except the $3 \mathrm{~cm}$ ones, which were acquired from Hamamatsu, were developed by Micron Semiconfuctor Limited in conjunction with the Ohio State Jniversity.

Fig. 6 illustrates a mounted $5 \mathrm{~cm}$ square detector plane. These planes consisted of a silicon wafer with 1000 diode strips of $50 \mu \mathrm{m}$ pitch and $35 \mu \mathrm{m}$ width etched onto it. Readout pads from the strips were alternated from side to side so that each side had a pad every $100 \mu \mathrm{m}$. Preamplifiers [11] were mounted directly on this circuit board to prevent noise generation by the capacitance in the lengthy signal path to the electronic racks. This permitted a signal-to-noise ratio of $20: 1$ to be achieved when the detectors were fully depleted at a reverse bias of $50 \mathrm{~V}$.

Since instrumenting all lines would not permit compact packaging along the beam line, a graded readout scheme was implemented as shown in table 3 . The success of this scheme, which ensured that more lines were read out in the central high density track regions than further out, relied on the ability of the detectors to share charge capacitively via the floating lines in the regions where every line was not read out. This charge sharing capability allowed positions to be interpolated to less than the readout spacing and reduced the num. ber of readout channels and therefore the number of preamplifiers and heat dissipation from each plane. Total lines read out were 8352 (6912 in first run).

The detectors were water-cooled using copper plates that had holes cut out to accommodate the preamplifiers. The back of each plate had rectangular channels into which were soldered the copper tubes circulating the cooling water. The total thickness of each such package was $7 \mathrm{~mm}$. The packages were combined using rigid plastic ring spacers and slightly wider flexible O-rings. The whole assembly was compressed with the O-rings squeezing down to the plastic ring thickness thus forming a sealed volume surrounding the silicon through which dry nitrogen gas was circulated. The
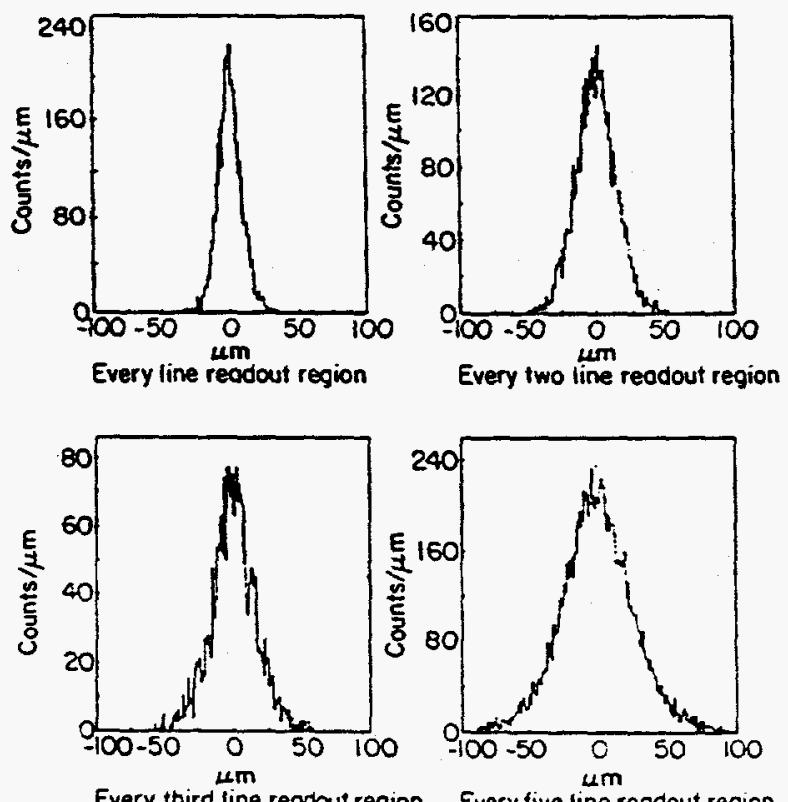

Fig. 7. Residuals in VSSDs. 
detectors were mounted in an aluminum frame with precision Thomson rods and the frame was sealed within an aluminum housing and was bolted to a pedestal resting on the same granite block as the beam SSDs and the target mover. Thin aluminum windows were placed at the entrance and exit of the housing [12].

Fig. 7 plots the residuals of tracks passing through each type of readout region in the $5 \mathrm{~cm}$ square detector. The central region of the detector achieved a resolution of $8.8 \mu \mathrm{m} \mathrm{mms}$ - better than the $14 \mu \mathrm{m}$ resolution expected from the strip spacing alone. This is due to diffusion charge sharing between adjacent lines.

In the first run all 18 planes were of the $5 \mathrm{~cm}$ square type. The larger downstream counters in the second run improved the solid angle acceptance and tracking efficiency. Performance of the spectrometer microstrip detectors was trouble-free throughout both runs. The detectors had an overall efficiency of $96 \%$; the $4 \%$ inefficiency was mainly due to shorted amplifiers or disconnected strips.

\subsection{The spectrometer drift chambers}

The downstream arm of the spectrometer consisted of 11 "vector" drift chambers - an equivalent of 55 conventional chambers since each chamber had five sense wires along the longitudinal direction. Multiple sampling in the chambers allowed left-right ambiguities to be resolved using track vector (slope) information. The chambers had an active area of $1.7 \times 1.7 \mathrm{~m}$ and were arranged in three views: the $x$. view readout was along the horizontal magnet bend plane, and the $u$ and $v$ readout directions were rotated $\pm 30^{\circ}$ with respect to the horizontal. The most downstream plane yielded a transverse acceptance of 240 mrad.

The drift chambers could resolve a track's position to $50 \mu \mathrm{m}$ rms per chamber for beam tracks, but typically achieved a $65 \mu \mathrm{m}$ rms error in the "busy" environment of Tevatron primary hadronic interactions. Adjacent and nearly parallel particle tracks as close as 600 $\mu \mathrm{m}$ could be separated by these chambers.

The design of the chambers is illustrated in fig. 8a. Five position samples were made at $1.02 \mathrm{~cm}$ intervals along the beam direction making vectors for the portions of the tracks in the chamber. As the figure shows, two different cell sizes were employed. Ten "fine" cells covered the central $50.8 \mathrm{~cm}$ of the detector where the track density was the highest and the slopes were generally less than 100 mrad. Six "coarse" cells on each side of the central region covered the outer portion of the chamber, where track density was low, track slopes were relatively large, and two-track separation capability was not a major concern. The fine cells had a maximum drift distance of $2.54 \mathrm{~cm}$ and the five position samples were obtained by adding the signals from pairs of sense wires spaced at $5.1 \mathrm{~mm}$ intervals and separated by large

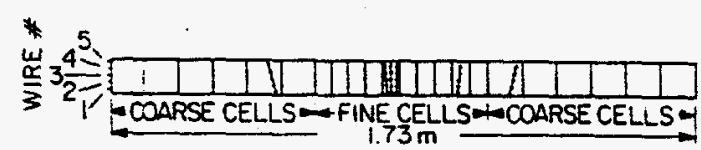

(a)
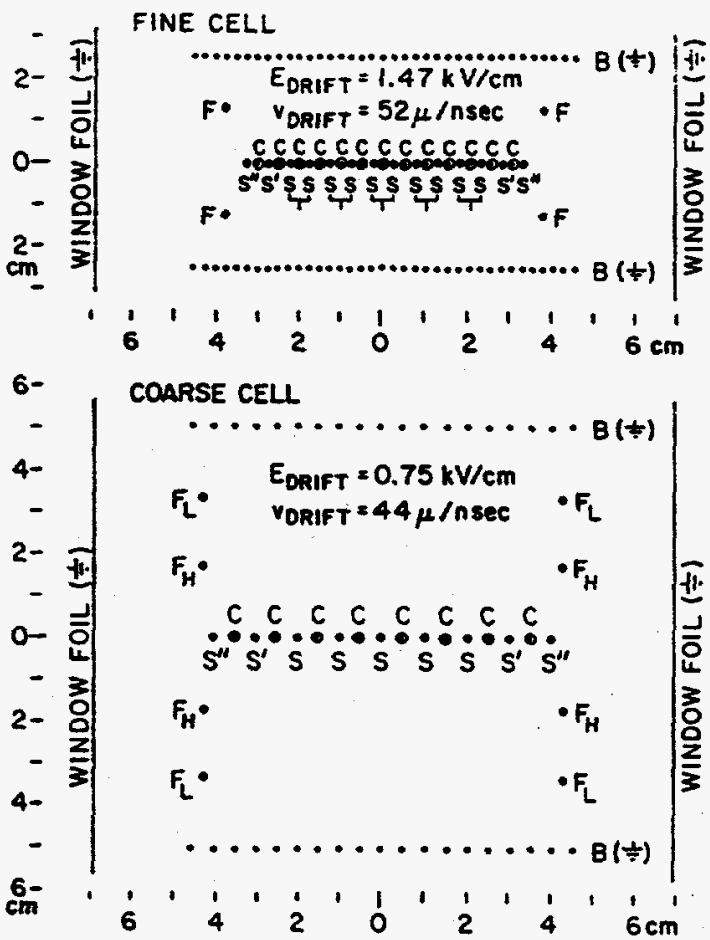

(b)

Fig. 8. Spectrometer drift chiambers; (a) cell structure and (b) wire configuration in fine and coarse cells.

diameter cathode wires. The coarse cells had a maximum drift distance of $5.08 \mathrm{~cm}$. These cells were similar to the fine cells except they had only five nonpaired sense wires spaced at $10.2 \mathrm{~mm}$ intervals. The combination of short drift distances along with the excellent transmission-line properties of the sense-cathode pairs and the signal summing of sense pairs yielded very short

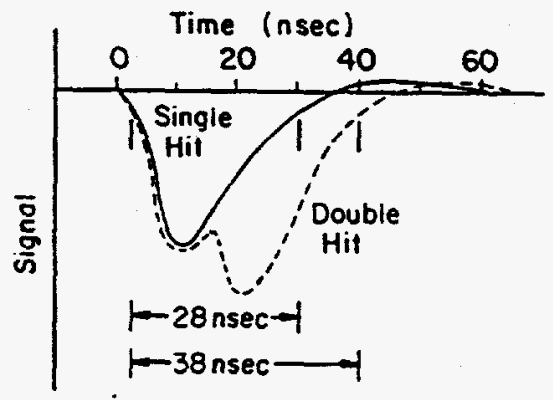

Fig. 9. Time-over-threshold pulse shapes of spectrometer drift chamber. 
Table 4

Wire parameters for spectrometer drift chamber fine and coarse cells

\begin{tabular}{llccl}
\hline Wire type & $\begin{array}{l}\text { Material } \\
\text { (Au plated) }\end{array}$ & $\begin{array}{l}\text { Diameter } \\
{[\mu \mathrm{m}]}\end{array}$ & $\begin{array}{l}\text { Tension } \\
{[\mathrm{g}]}\end{array}$ & $\begin{array}{l}\text { Potential } \\
{[\mathrm{V}]}\end{array}$ \\
\hline Fine S & W & 30 & 105 & 5375 \\
Fine $S^{\prime}$ & Al & 250 & 350 & 4670 \\
Fine S' $^{\prime \prime}$ & Al & 250 & 350 & 5375 \\
Fine C & Al & 250 & 350 & 3495 \\
Fine F & Al & 160 & 130 & 3145 \\
Fine B & Al & 160 & 130 & 0 \\
Coarse S & W & 30 & 80 & 5780 \\
Coarse S' & Al & 250 & 350 & 5070 \\
Coarse S" & Al & 250 & 350 & 5780 \\
Coarse C & Al & 250 & 350 & 3610 \\
Coarse F & Al & 160 & 130 & 1790 \\
Coarse F & Al & 160 & 130 & 4050 \\
Coarse B & Al & 160 & 130 & 0 \\
\hline
\end{tabular}

pulses (25-30 ns full width base) of a quite uniform length in the fine cells. These uniform short pulses allowed for the inference of unresolved nearby tracks from the time-over-threshold (TOT) recording electronics as shown in fig. 9.

Fig. $8 \mathrm{~b}$ displays the configuration of the wires in both the fine and coarse cells. The physical parameters of the chamber components, such as wire diameters, are described in table 4. Sense wires were made of goldplated tungsten, while all other wires were made of gold-plated aluminum. Voltages on the field shaping

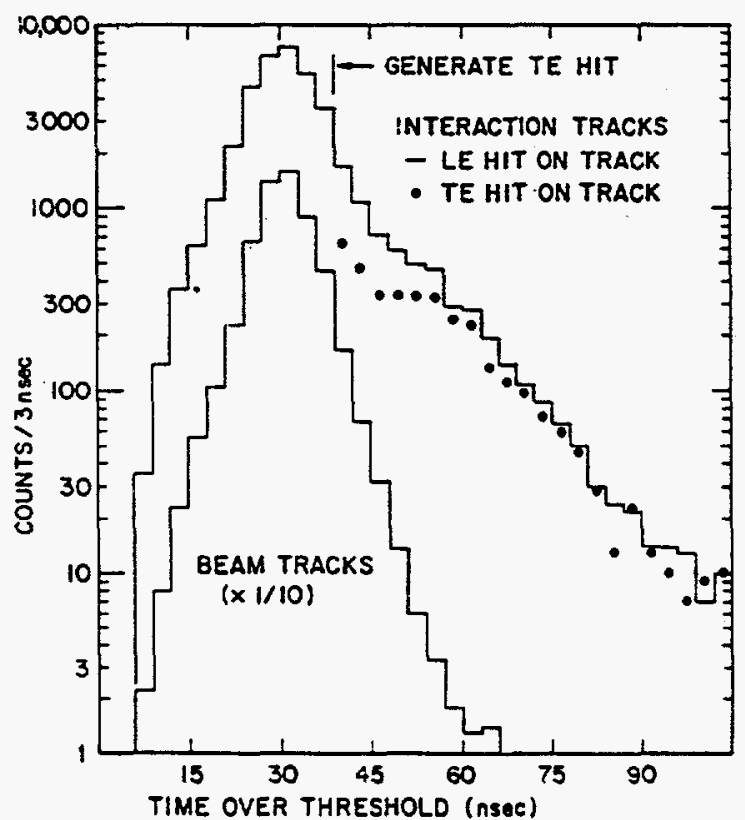

Fig. 10. Spectrometer drift chamber time-over-threshold distributions for beam and interaction triggers.

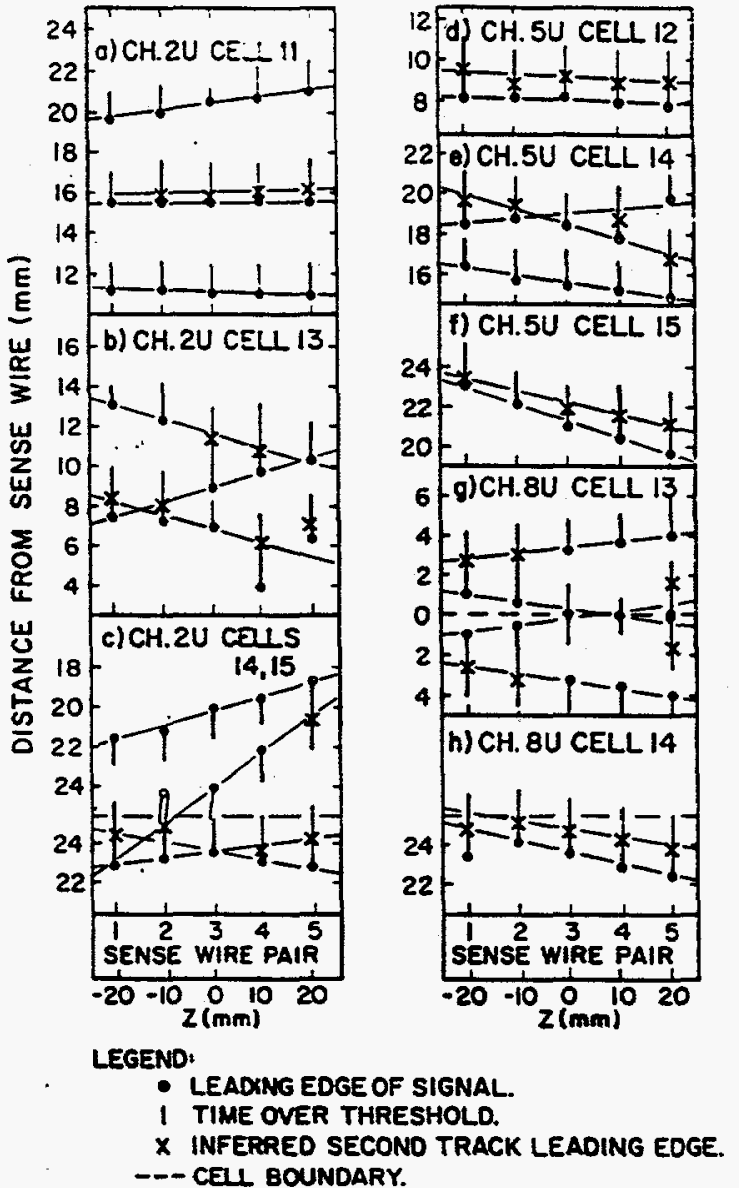

Fig. 11. Eight segments of tracks from an event in the spectrometer drift chambers.

wires ( $S^{\prime}, S^{\prime \prime}$ and $F$ in fig. $8 b$ ) were designed to give a uniform drift field. The large diameter of the cathode wires was chosen to keep the surface field low (8.7 $\mathrm{kV} / \mathrm{cm}$ ) thus minimizing problems with secondary emission.

Signals were amplified and discriminated using commercially available low-impedance amplifiers (LeCroy Research 2735A) prior to readout by LeCroy Research 1879 Fastbus TDCs. These TDCs generated leading and trailing edge times (LE and TE) for all pulses and preserved TOT information. For the coarse cells the TDCs were run at $334 \mathrm{MHz}$ instead of the $500 \mathrm{MHz}$ maximum in order to accommodate the longer drift time.

The chambers were run with a mixture of $49.5 \%$ 49.5\%-1.0\% argon-ethane-isopropanol at one atmosphere. (Isopropanol was substituted in the second run for ethanol because variation in its concentration had less effect on the drift time.) During operation, the high-voltage supplies were ramped between beam spills. The gains of all the sense wires in a chamber remained 


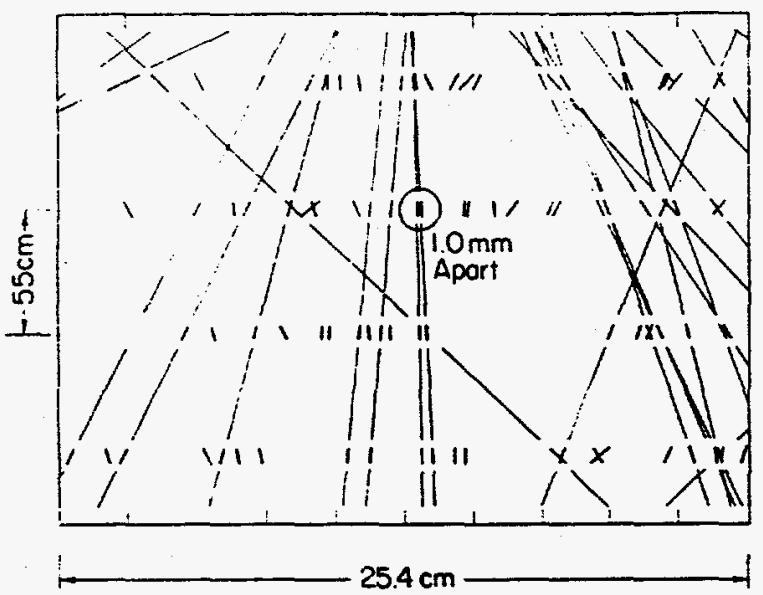

Fig. 12. Spectrometer drift chamber event illustrating resolution of nearly collinear tracks.

uniform to $\pm 15 \%$ when operated at the same voltage. Operation was relatively stable during the run.

TOT distributions for fine cell hits from single-beam track triggers and high-multiplicity interaction triggers are shown in fig. 10. The beam track TOT distribution has a spread of $13 \%$ rms, with negligible tails. Interaction tracks have a spread of $\mathbf{1 8 \%}$, with a long tail due to unresolved second hits. The analysis software generates a second hit from the trailing edge of the pulse if the TOT is more than 9 ns longer than the expected TOT value (arrow in fig. 10), corresponding to $0.47 \mathrm{~mm}$ in spatial separation. This cut increases the number of hits by $18 \%$, half of which are real and appear on fitted tracks found by analysis software.

Fig. 11 shows eight segment samples from one event which indicate how essential the trailing edge hits are for successful reconstruction. In each segment, at least one track would be lost if not for the trailing edge information. This figure also illustrates that crossing tracks are quite common. Fig. 12 displays cleanly resolved nearly parallel tracks with an average separation of $0.64 \mathrm{~mm}$.

Ten chambers were used in the first run. For the second run an 11th chamber was added to this grouping, and a 12th was placed further downstream to provide a link-up to the muon spectrometer. The spatial resolution was $110 \mu \mathrm{m}$ rms per wire pair $(50 \mu \mathrm{m}$ per chamber) for beam particles, and $140 \mu \mathrm{m}$ rms per fine cell wire pair ( $65 \mu \mathrm{m}$ per chamber) for tracks originating from interactions. Trailing edge hits and coarse cells had a resolution half as good as leading edge hits. These resolutions corresponded to slope errors over the chambers of 30-35 $\mu \mathrm{rad}$ for the fine cell beam tracks, and 40-45 $\mu \mathrm{rad}$ for the fine cell interaction tracks. Coarse cell leading and fine cell trailing edge hits had double that error on the slopes.

\subsection{The spectrometer magnet}

The spectrometer magnet [13] was used to determine the momentum of charged tracks and swept out low momentum particles. Its vertical dipole gap was set at $50.8 \mathrm{~cm}$ and shaped pole pieces were added to increase the magnetic field without reducing the geometric acceptance of the experiment. The price paid was a very nonuniform field which required detailed mapping.

The field was measured in situ at orthogonal grid points every $12.7 \mathrm{~mm}$ along the beam direction and every $25.4 \mathrm{~mm}$ in the transverse directions, extending one meter beyond the magnet both upstream and downstream. Maxwell field equation constraints were used to extrapolate into unmeasured regions. The total $\int B \mathrm{~d} l$ along the beam line was determined to be $1.12 \mathrm{Tm}$ at the operating current of 2400 A yiejding an effective transverse momentum kick of $0.336 \mathrm{GeV} / c$.

Error analysis using the thin lens approximation gave a momentum measurement error of:

$\sigma_{p} / p=\sqrt{(0.01)^{2}+(0.00023 p)^{2}} \quad(p$ in $\mathrm{GeV} / c)$,

where the first term is estimated from multiple scattering and the second term is due to errors in the slope determinations and is a measured quantity (18\% error for $800 \mathrm{GeV} / c$ beam particles). The second term is consistent with the expected slope measurement errors of $70 \mu \mathrm{rad}$ in the solid state microstrip detectors and $30-35 \mu \mathrm{rad}$ in the spectrometer drift chambers for beam tracks, but is as high as $\left(0.0004 p^{2}\right)$ for wider angle tracks passing through the coarser segmentation areas of these detectors.

\subsection{Time-of-flight counters}

The time-of-flight (TOF) system, along with momentum information from the charged particle spectrometer, was used to assign particle identifications to lowenergy charged particles. This system was the only component of the experiment that could directly identify charged hadrons. It consisted of $4838.1 \mathrm{~mm} \times 38.1$ $\mathrm{mm} \times 1.68 \mathrm{~m}$ long scintillators placed $4.5 \mathrm{~m}$ downstream of the target (4.1 $\mathrm{m}$ in first run) and arranged vertically side by side in a Unistrut frame so that their total horizontal dimension measured $1.88 \mathrm{~m}$. Fig. 13 shows beam's eye and elevation views of the TOF system. The scintillators were viewed by Amperex XP2230 photomultiplier tubes at both ends. The tube signals were split in a 5:1 ratio; the larger signals were immediately discriminated, then delayed and recorded in Camac time-to-digital converters (TDCs), while the smaller signals were recorded in Fastbus analog-todigital converters (ADCs). The TDCs started from the edge of the trigger counter pulse, whose time resolution was typically 600 ps. In practice, delays relative to stiff 


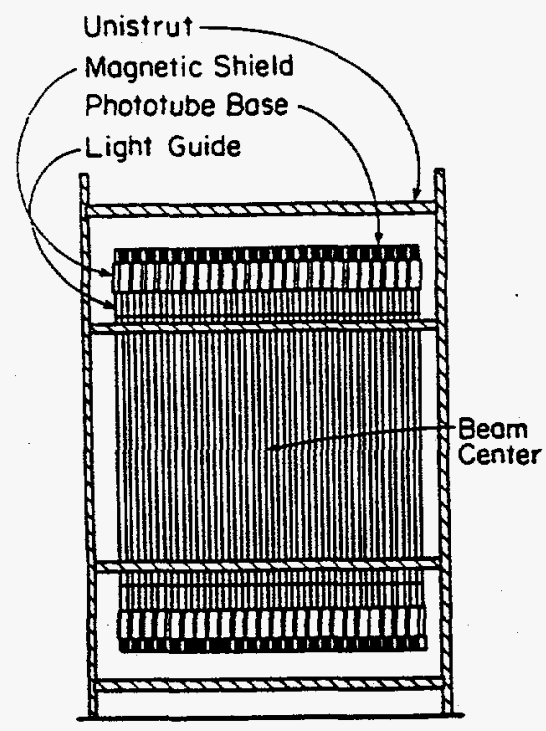

Beam's Eye View

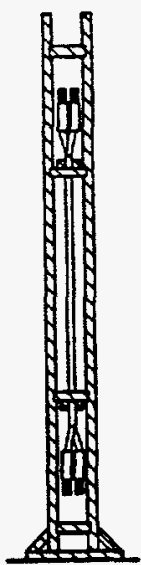

Side View
Fig. 13. Time-of-flight hodoscope.

high momentum tracks in an event, rather than absolute start time signals, proved to be most useful for timing. The 105 ps resolution of the TOF counters together with the flight time from the target gives an upper limit on the energy of the particles to be separated. For a two standard deviation ( $95 \%$ confidence level) difference in the flight time:

pion-kaon separation $E=2.8 \mathrm{GeV}$,

kaon-proton separation $E=5.3 \mathrm{GeV}$.

At higher energies, the particles cannot be unambiguousiy identified.

The resolution of the TOF system was determined by taking events with at least four particles having fit start times, refitting the start time using three of the particles; and comparing the fourth time to that fit (actually, each photomultiplier tube was compared separately, giving a resolution per tube, rather than per track). The results of that fit are shown in fig. 14a. The width of this distribution corresponds to 1.3 times the resolution of a single tube, giving a single-tube resolution of 150 ps and a two-tube resolution of 105 ps, as quoted above.

The effectiveness of the TOF system was determined by looking at the fraction of tracks it was able to identify. (All TOF performance data quoted were derived from the first run.) The average number of tracks per event which were matched in the magnet, and therefore had the most reliable momentum measurement, was 12.6. A cut was then imposed allowing only a single hit in each counter, leaving 7.0 tracks per event. Of these, 4.3 tracks had the measured top and bottom times agreeing within errors, and a pulse height which was consistent with a single minimum-ionizing particle traversing the counter. The loss here was from unreconstructed tracks, including backsplash from the liquid argon calorimeter, and inefficiencies in the apparatus and electronics. A start time was calculated for each of these tracks, and the most likely hypothesis (pion, kaon, or proton) was selected. Any track with a start time differing by more than a few standard deviations from the others was rejected. This left an average of 3.9 tracks per event, which were combined to fit an overall start time. On average, $46 \%$ of the events at this stage had fewer than three tracks left, a number considered insufficient to yield a reliable start time; these could not be fitted. Of the final fitted tracks, $46 \%$ had a momentum greater than $5 \mathrm{GeV} / \mathrm{c}$ and could not be identified at the two standard deviation level; $34 \%$ had a momentum less than $3 \mathrm{GeV} / c$ and could be identified as pion, kaon, or proton; and 20\% had a momentum between 3 and $5 \mathrm{GeV} / c$ and could be identified either unambiguousiy as protons or ambiguously as pions or kaons. Another way of expressing these results is that generally. one track per event could be identified in some way. Fig. 14b shows a mass plot of all tracks with momentum below $2.5 \mathrm{GeV} / c$ fitted to the start time. The width
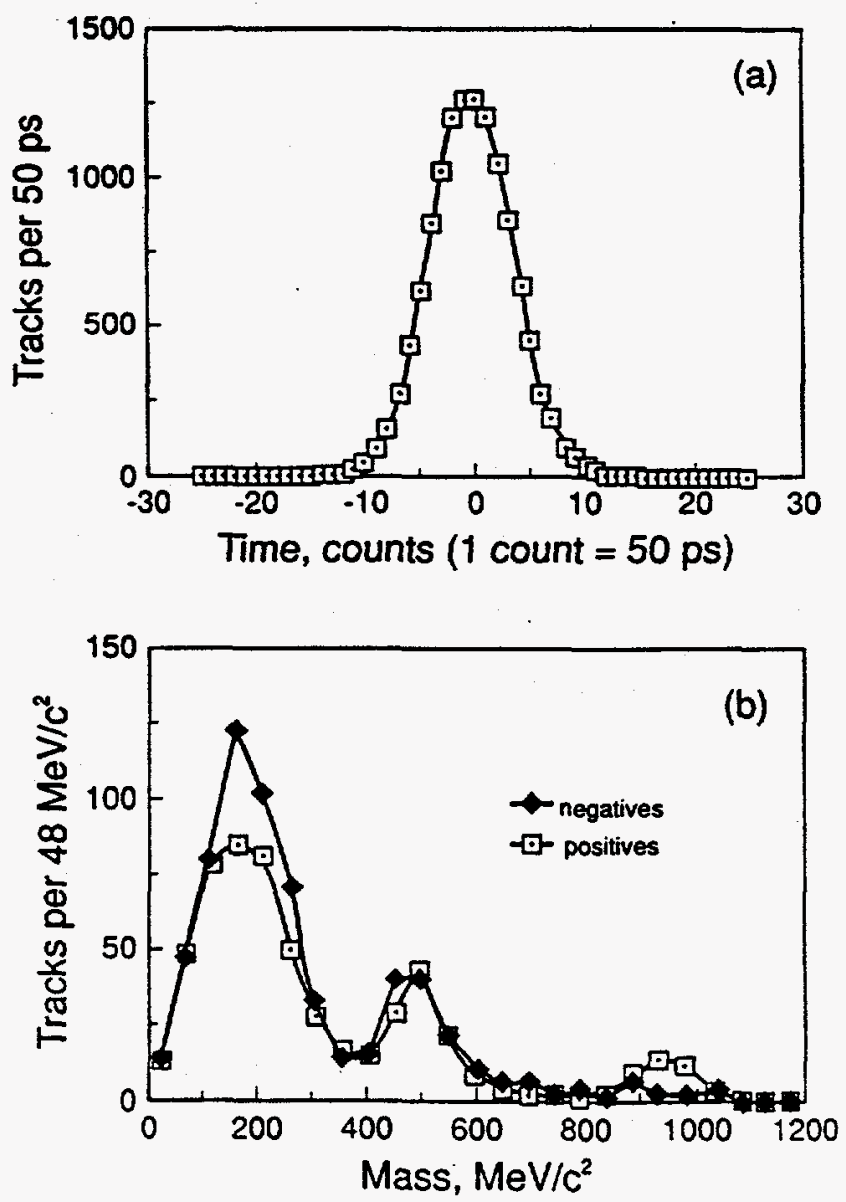

Fig. 14. Time-of-flight resolution; (a) time resolution and (b) mass resolution. 
of the mass peaks is consistent with the resolution quoted above, and the absence of antiprotons relative to protons is as expected.

\subsection{Calorimeters}

The experiment contained two calorimeters. Downstream of the TOF scintillator wall was a lead and iiquid argon calorimeter (LAC) which was capable of localizing electromagnetic showers and determining incident $\gamma$ ray and electron energies. $\gamma$ ray pairs were used to reconstruct neutral pions. The LAC was followed by a hadron calorimeter ( $\mathrm{HadCal}$ ), which consisted of steel plates alternating with planes of proportional tubes. Two-dimensional readout gave the position of the hadronic shower and measurement of total charge deposited permitted an estimate of the hadronic energy.

\subsubsection{Liquid argon calorimeter (LAC)}

The lead and liquid argon calorimeter was centered on the beam line $5.5 \mathrm{~m}$ downstream of the emulsion target. Because of the short distance between the target and the LAC, pattern recognition in the high shower multiplicity environment was our primary concern. Electron and $\gamma$ ray profiles were measured with a transverse segmentation finer than the shower width. This allowed known shower shapes to be used to separate overlapping showers in the highly active central region of the detector. The spatial and energy information permitted unambiguous matchups between the $x$ and $y$ strips, making two-dimensional shower reconstruction possible.

The active area of the LAC was $1.625 \mathrm{~m} \times 1.625 \mathrm{~m}$. it was segmented in three longitudinal sections as illustrated in fig. 15. The front and middle sections were used for electron and $\gamma$ ray detection, while the back section was used primarily to reject hadronic showers. The front section was used in the pattern recognition to separate overlapping showers using their transverse profiles. Fig. 15 also illustrates the "generic" sandwich composition of a cell. There were 15 front cells consisting of $2.39 \mathrm{~mm}$ of lead, $2.54 \mathrm{~mm}$ of liquid argon, and two $1.59 \mathrm{~mm}$ thick back to back G-10 circuit boards followed by another $2.54 \mathrm{~mm}$ of liquid argon. The lead acted as the high-voltage electrode of the cell, while the G-10 circuit boards were the low-voltage electrodes. The middle section consisted of 18 cells identical in construction to the front ones. The 7 cells in the back section had lead of thickness $4.67 \mathrm{~mm}$. The radiation lengths were $7.2,8.6$, and 6.2 for the front, middle, and back sections respectively.

Charge was collected on the low-voitage electrodes etched on the copper clad G-10 boards in the cells. These hoards had 3 geometries: $x$ strips, $y$ strips, and full pad readout. The $x$ and $y$ strips were $5.1 \mathrm{~mm}$ by $1.63 \mathrm{~m}$ long, but the central strips were split into a 163
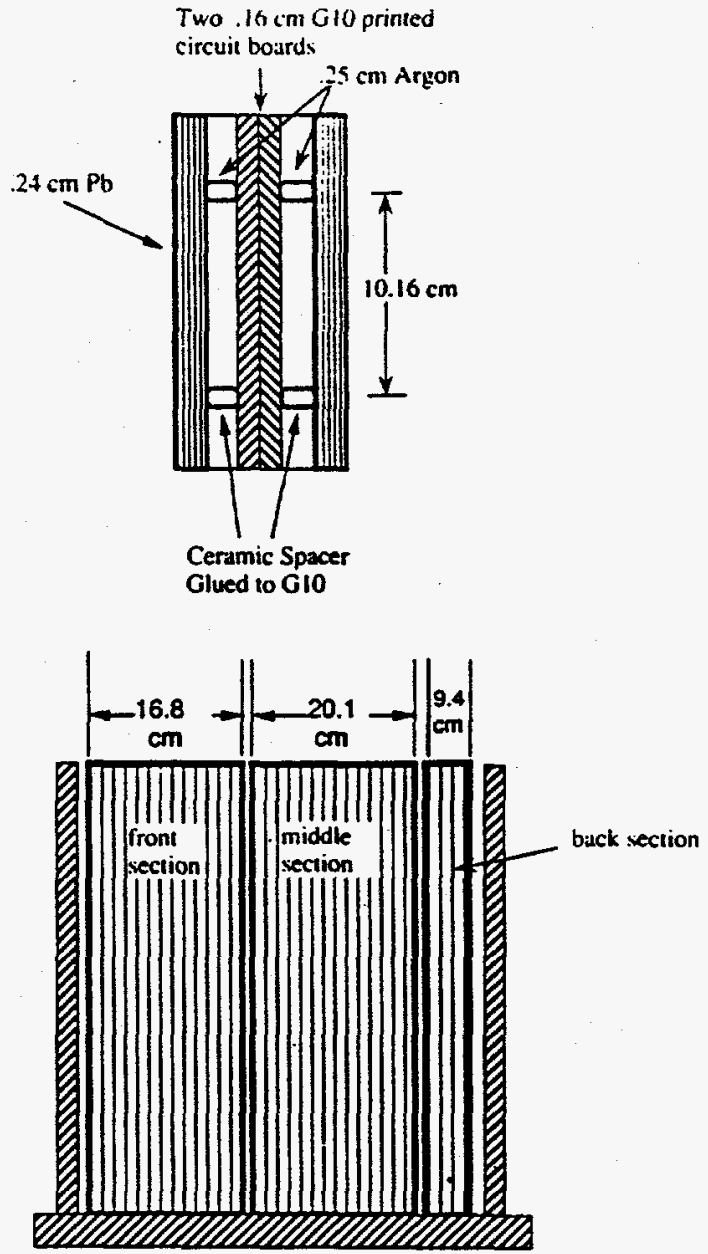

Fig. 15. Lead-liquid-argon calorimeter cell structure and inner core segmentation.

$\mathrm{mm} \times 163 \mathrm{~mm}$ region of $2565 \mathrm{~mm} \times 20 \mathrm{~mm}$ rectangular pads. Fig. 16 shows the full pad geometry. All pads, including those in the central region of the strip readout geometry, were read out with copper strips on the back of the boards. The strips were electrically connected to the pads with soldered rivets which passed through the boards. The front and middle sections of the LAC had $x$ PAD, $y$ PAD, $x-y$ sampling, while the back section was read out entirely via pads. The whole assembly rested on a steel plate which was suspended in a liquid argon cryostat by four thin-walled steel pipes attached to the cryostat lid. The cryostat was built of stainless steel and was insulated with $23 \mathrm{~cm}$ thick foam.

The 3660 pad and strip readout channels were read through charge sensitive preamplifiers and shaping amplifiers into LeCroy 1885 ADCs. All channels were calibrated weekly by injecting a fixed charge into all read-out preamplifiers. Cross talk was measured to be $1-2 \%$, and the relative gains were measured to a precision of $1-2 \%$. 


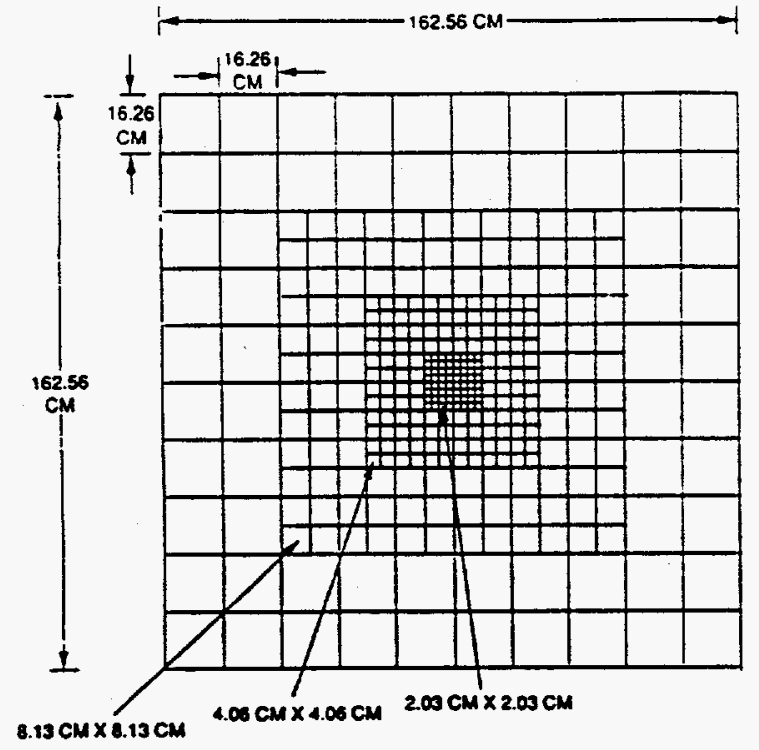

Fig. 16. Lead-liquid-argon pad readout segmentation.

Fig. 17 shows a typical event in the LAC. $x$ and $y$ strip pulse heights are plotted along the inner border. Pad hits are represented by circles whose diameter is proportional to the energy deposited in the pad. The event illustrates the separation of showers in the central region by the use of pad and shower shape information.

The energy error depended strongly on the shower density. This effect is shown in fig. 18 where $5 \mathrm{GeV}$ sowers generated by the EGS simulation [14] were superimposed on emulsion data events. There was clearly a strong dependence of the energy resolution on energy from overlapping showers. This effect was especially pronounced in the middle section of the detector (6-14

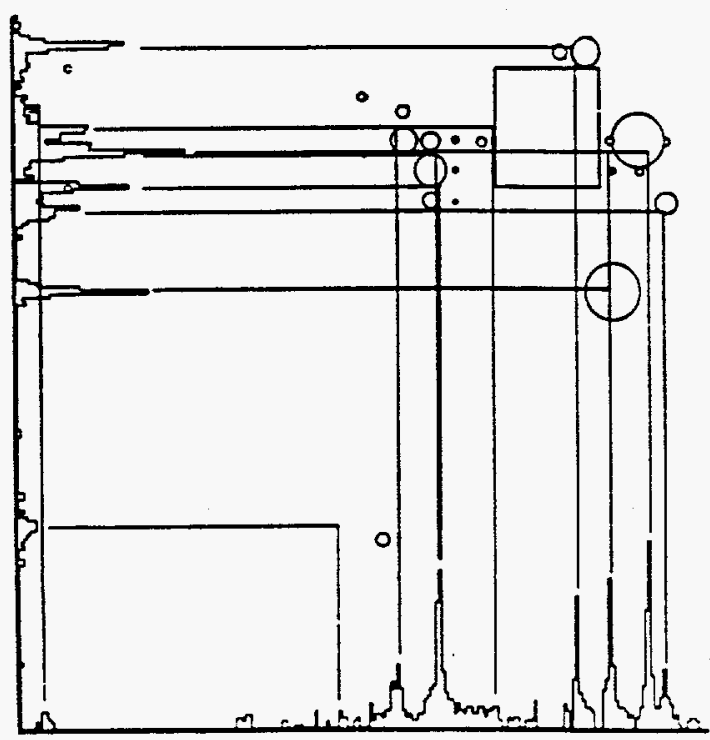

Fig. 17. Typical event in the lead-liquid-argon calorimeter.

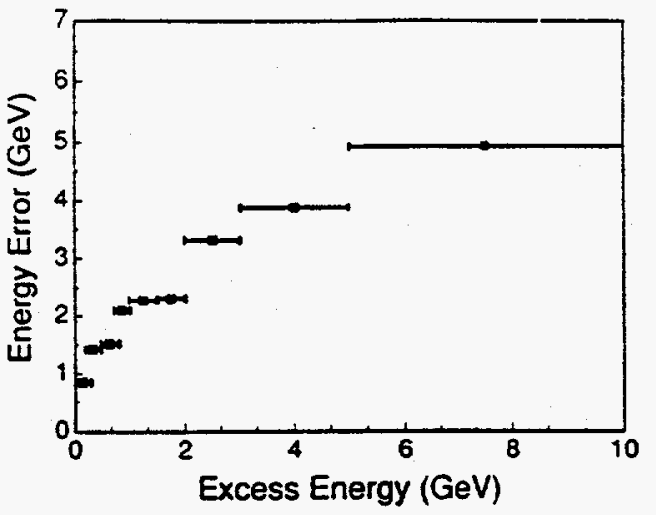

Fig. 18. Energy error versus shower densily.

radiation lengths) where the showers spread substantially. This was the dominant source of energy error overall. Energy resolution, which was $(15 / \sqrt{E}+1.2) \%$ in an electron beam ( $E$ in $\mathrm{GeV}$ ), increased to $25 \% / \sqrt{E}$ in data events. The LAC position resolution in an electron beam was $1 \mathrm{~mm}$ rms and did not depend strongly on shower overiaps.

The high shower density (averaging 15 reconstructed showers/event) also affected neutral pion identification. Mass distributions with diphoton energies below $20 \mathrm{GeV}$ and low transverse momentum ( $<500 \mathrm{MeV} / c$ ) were dominated by combinatorial background. The diphoton mass distribution for neutral pion candidates of energy greater than $30 \mathrm{GeV}$ is shown in fig 19. In kinematically fitting our events, which typically had low energy (around $10 \mathrm{GeV}$ ) neutral pions, we had the additional constraint of transverse momentum balance at the decay vertex. This constraint reduced the background to tolerable levels $(<15 \%)$ in one fifth of the events with candidate neutral pion fits [15].

Fig. 20 shows a plot of $E / P$ for eloctron candidates using the pad view energy. This view had the smallest error in high shower density events. The electrons, usually from photon conversions in the emulsion, had an average energy of $5 \mathrm{GeV} / c$. The LAC identified elec-

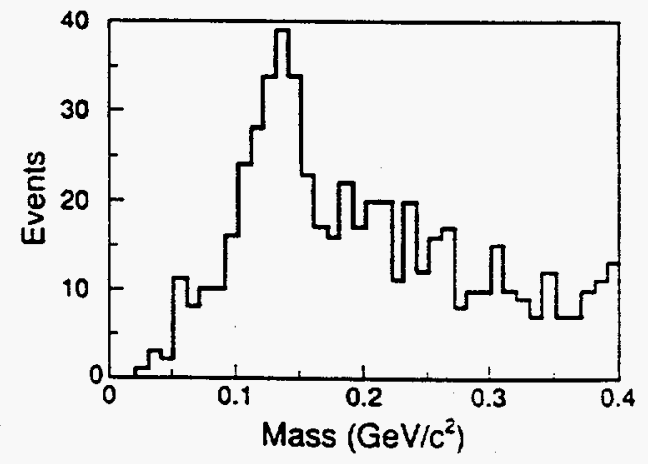

Fig. 19. Mass distribution for neutral pion candidates of energy greater than $30 \mathrm{GeV}$. 


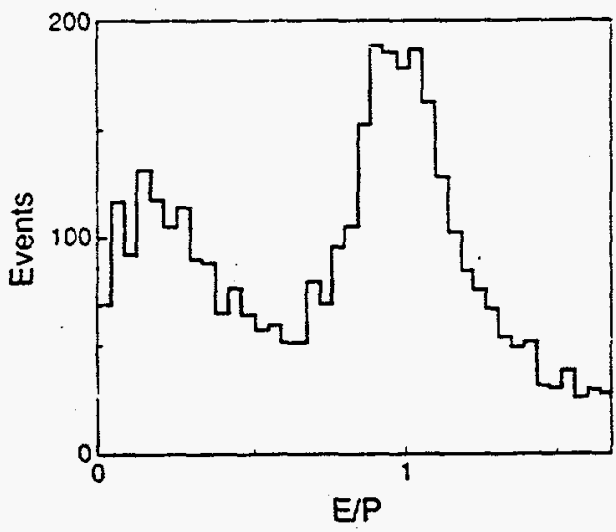

Fig. 20. $E / p$ for electron candidates.

trons with an efficiency of $70 \%$ and hadron misidentification of $1.5 \%$.

\subsubsection{Hadron calorimeter ( $\mathrm{HadCal)}$}

The hadron calorimeter was located between the liquid argon calorimeter and the muon detector system and provided rough position and energy measurements for hadrons. The first few planes of this calorimeter also ranged out any electromagnetic showers that punched through the liquid argon calorimeter.

The HadCal consisted of 16 planes of resistive plastic proportional tubes with pad readout alternating with 16 iron plates. The tube and pad readout geometry covered an active area of $2.34 \mathrm{~m} \times 2.34 \mathrm{~m}$ with a varying degree of segmentation, and allowed for unambiguous two-dimensional localization of a hadron shower to within a single plane. The $5 \mathrm{~cm}$ thick steel plates measured $2.44 \mathrm{~m}$ vertically and $3.05 \mathrm{~m}$ horizontally and provided a total of 4.8 hadronic interaction lengths. Energy was measured by the proportional tube sampling, while position was resolved with both pad and tube readout [16].

Each active plane of the detector was made of three modules, the center module being more finely segmented than the two side ones. The former had 48 and the latter 50 tubes each with a center-to-center spacing of $1.60 \mathrm{~cm}$. the smallest pad dimensions were 3.20 $\mathrm{cm} \times 6.40 \mathrm{~cm}$ in the center module. The modules were constructed by epoxying $1.6 \mathrm{~mm}$ thick copper-clad G-10 boards on opposite sides of the plastic proportional tubes. The G-10 boards had identical patterns of rectangular pads etched in them on the sides adjacent to the tubes and the overlapping pads on both sides of the tubes were electronically ganged together. Readout lines were etched onto the other sides of the boards and were electrically connected to the pads by means of eyelets. Fig. 21 shows the pad pattern for one of the planes. Alternate planes were rotated at $90^{\circ}$. The tubes had an average resistivity of $10^{4} \Omega \mathrm{cm}( \pm 20 \%)$. They contained gold-plated tungsten wire $51 \mu \mathrm{m}$ in diameter, and were. operated at $1900 \mathrm{~V}(2100 \mathrm{~V}$ in the first run) in a gas mixture of $48.6 \%$ argon, $48.6 \%$ ethane, and $2.8 \%$ alcohol at atmospheric pressure. Pads were ganged 8 planes deep in each view for a total of 1016 readout channels, while tubes were ganged two planes deep in each view for a total of 1152 channels.

Muon tracks were used to investigate gain uniformity and to check the pad readout. An efficiency of $74 \%$ per layer averaged over the four readout layers in each view was obtained using high voltage of $2.2 \mathrm{kV}$. The tube and pad signals were measured to be the same for individual tracks within the noise performance of the amplifiers. Tests up to $2.5 \mathrm{kV}$ with minimum ionizing tracks showed that charge flow along the tubes from one pad to an adjacent pad was less than 12\%. The tube and pad pulse height distributions were found to be uniform over the active area of the detector to $\pm 7 \%$. Fig. 22 shows the response to a test beam having a mean energy of $17.6 \mathrm{GeV}$. The $2.1 \mathrm{kV}$ high voltage used in the first run caused power supply trips during the occasional higher instantaneous beam rates and for the second run charge-sensitive amplifiers were installed, permitting operation at $1.9 \mathrm{kV}$. This change, together with improved beam intensity control resulted in a very stable detector during the second run.

While under test beam conditions HadCal achieved an $\mathrm{rms}$ position resolution of $0.5 \mathrm{~cm}$ and an energy resolution of $\sigma_{E} / E \leq 1.0 / \sqrt{E}(E$ in $\mathrm{GeV})$, during actual data taking the high particle multiplicity (on average, $\mathrm{HadCal}$ obtained 22 showers per event) and the compact geometry of the experiment made it difficult to identify isolated energy depositions unambiguously. Nevertheless, $\mathrm{HadCal}$ information proved useful in the fitting of some zero constraint events by indicating

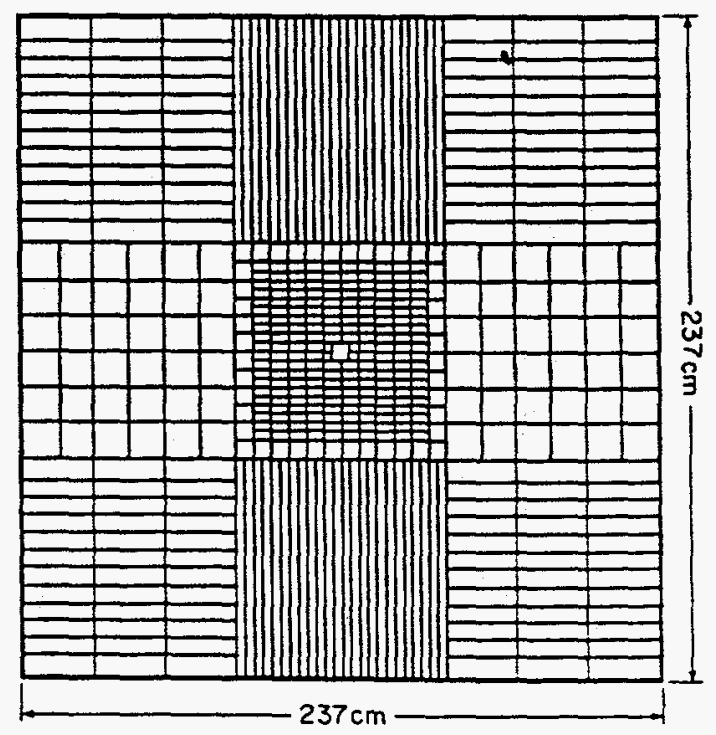

Fig. 21. Hadron calorimeter pad readout geometry. 


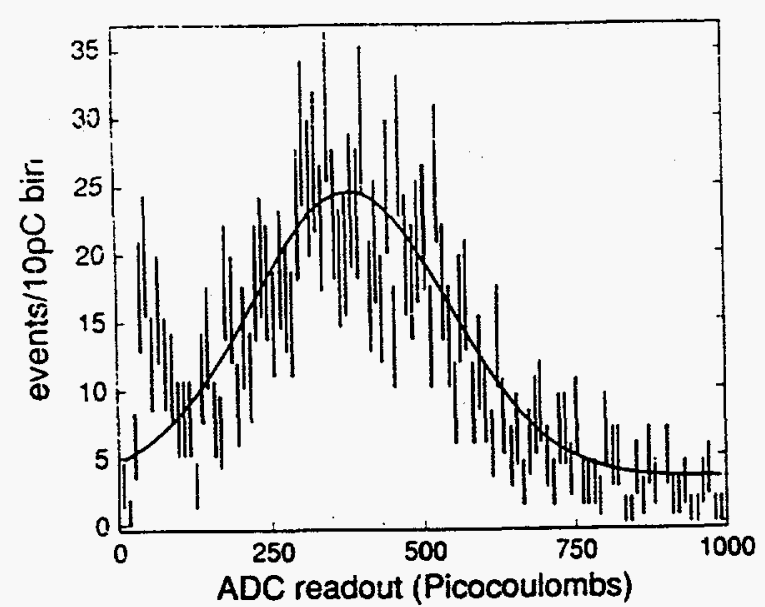

Fig. 22. Hadron calorimeter response to test beam of $17.6 \mathrm{GeV}$ inean energy. Errors are statistical. HV $=2.1 \mathrm{kV}$. The background at low energy is due to electron contamination and was not included in the solid line fit; $\sigma /$ peak $=0.43$ giving $\sigma_{E} / E$ $=1.8 / \sqrt{E}$. This is an upper limit since test beam was not monochromatic but had a $30 \%$ energy spread.

excess energy from missing neutrals in the predicted positions.

\subsection{Muon detection system}

Because the experiment triggered on a single muon from the decay of a heavy quark, the muon detection system [18] assumed an importance beyond that of mere particle identification. It was, in effect, an independent spectrometer with six drift chambers each upstream and downstream of an iron toroid magnet. The trigger was generated by a double wall of scintillation counters at the downstream end of the experiment, with one wall segmented using horizontal counters and the other using vertical counters. Extensive use of the muon spectrometer information was made in the off-line analysis, such as cutting on muon transverse momentum to enrich the heavy quark sample. Tracking and momentum information was used to link muon tracks to the upstream

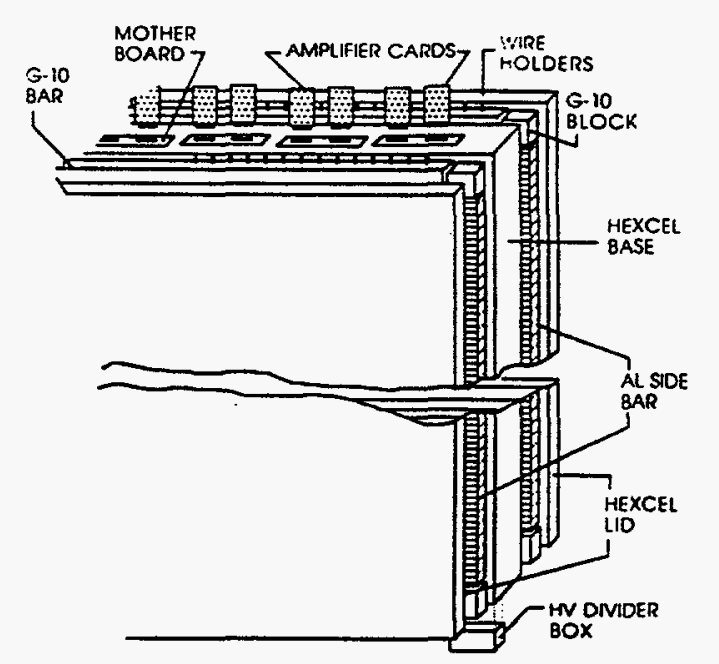

Fig. 23. Muon chamber structure.

spectrometer and ultimately to the target region. During the first run hadron "punch through" reduced muon track reconstruction efficiency somewhat by producing multiple hits in the chambers upstream of the toroid and complicating the combinatorics. The addition of more steel virtually eliminated this problem during the second run. The final muon filter steel positions are shown in fig. $I$ and the physical properties are listed in table 5, which indicates that the muon trigger energy threshold was $6 \mathrm{GeV}$. (It was $5 \mathrm{GeV}$ in the first run.)

\subsubsection{Muon spectrometer}

The muon spectrometer system had to have rather large transverse dimensions to match the acceptance of the upstream spectrometer. All its components were centered on a circular iron toroid $180 \mathrm{~cm}$ in radius, 133 $\mathrm{cm}$ deep, with a central hole of radius $12.7 \mathrm{~cm}$. The toroid was located $11.5 \mathrm{~m}$ downstream from the target, with its center $0.5 \mathrm{~m}$ above the beam axis in order to keep the hole away from the busiest muon region. The twelve $3 \mathrm{~m} \times 3 \mathrm{~m}$ drift chambers were oriented in three

Table 5

Ranging parameter of the spectrometer

\begin{tabular}{lccccc}
\hline $\begin{array}{l}\text { Spectrometer } \\
\text { component }\end{array}$ & $\begin{array}{l}\text { Density } x \\
\text { thickness } \rho \Delta z \\
{\left[\mathrm{~g} / \mathrm{cm}^{2}\right]}\end{array}$ & $\begin{array}{l}\text { Interaction } \\
\text { lengths }\end{array}$ & $\begin{array}{l}\text { Radiation } \\
\text { lengths }\end{array}$ & $\begin{array}{l}\text { Muon ionization } \\
\text { minimum }\left(I_{\mu}\right) \\
{\left[\mathrm{MeV} \mathrm{cm}^{2} / \mathrm{gl}\right.}\end{array}$ & $\begin{array}{l}\text { Energy loss } \\
\rho \perp z I_{\mu} \\
{[\mathrm{GeV}]}\end{array}$ \\
\hline LAC & 217 & 1.44 & 24.9 & 1.32 & 0.285 \\
HadCal & 630 & 4.78 & 45.5 & 1.48 & 0.932 \\
Steel I & 315 & 2.39 & 22.8 & 1.48 & 0.466 \\
Steel II & 504 & 3.82 & 36.4 & 1.48 & 0.746 \\
Torcid & 1060 & 8.03 & 76.4 & 1.48 & 1.568 \\
Steel III & 323 & 2.45 & 23.3 & 1.48 & 0.478 \\
Steel IV & 880 & 6.67 & 63.6 & 1.48 & 1.302 \\
Total & & 30 & 293 & & 5.78 \\
\hline
\end{tabular}




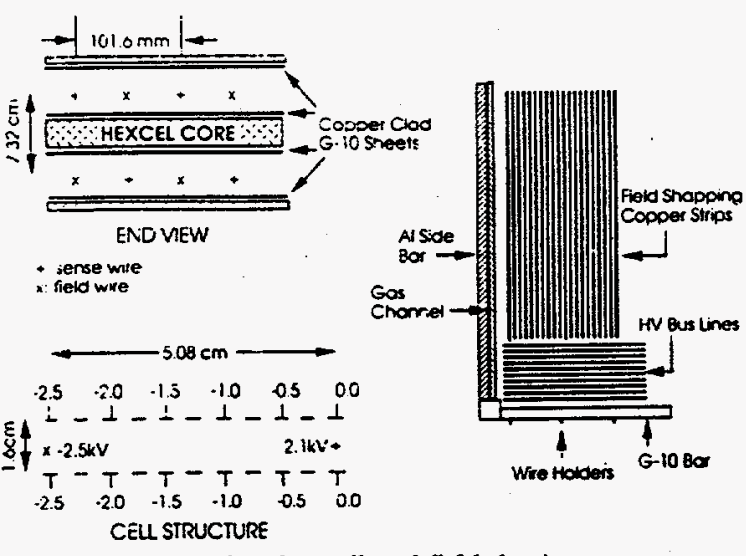

Fig. 24. Muon chamber cell and field shaping structure.

views: one view was aligned vertically $(y)$, and two views ( $u$ and $v$ ) were rotated $\pm 12^{\circ}$ with respect to the horizontal plane. The chambers were operated with an equal argon-ethane mixture at one atmosphere.

Fig. 23 illustrates the overall construction of these chambers, while fig. 24 shows a cell in detail. Each chamber had two $1.6 \mathrm{~cm}$ wide gaps separated by a central plane. The gaps were offset by one half of the transverse cell size $(5.08 \mathrm{~cm})$ relative to one another to resolve left-right ambiguities. Copper field shaping strips of $0.25 \mathrm{~cm}$ width were etched onto G-10 boards and placed on each side of the central plane. Cathode and sense wires were made of gold-plated tungsten 50 and $30 \mu \mathrm{m}$ in diameter and were strung to tensions of 100 and $60 \mathrm{~g}$ respectively. The use of Hexcel sheets, which were made of aluminum honeycomb sandwiched between.thin aluminum sheets, enabled the chambers to maintain mechanical rigidity with a minimum of material.

For well-collimated muon tracks the upstream and downstream track segments projected to the toroid center had a projected spatial resolution of $400 \mu \mathrm{m} \mathrm{ms}$ on average.

A computed field map [17] agreed with radial Hall probe measurements to $2 \%$. A least squares fit made to the calculated field to obtain the toroidal field as a function of radius indicated that the field ranged from 2.2 to $1.7 \mathrm{~T}$.

Momentum was determined by:

$p=0.03 \times B(r) \times \Delta Z / \delta \theta \quad(\mathrm{GeV} / c)$,

where:

$B(r)=$ fit field as a function of the toroid radius $r$ (in kilogauss),

$\Delta Z=$ the toroid thickness $(1.3 \mathrm{~m})$,

$\delta \theta=$ the angular deflection of the track in radians.

The resolution was measured by looking at the difference in momentum as determined by the two spectrometers (after correcting for energy loss in the inter- vening material) for a well-collimated sample of muon tracks. The result

$\sigma_{p} / p=\sqrt{(0.31)^{2}+(0.008 p)^{2}} \quad(p$ in $\mathrm{GeV} / c)$

has energy loss fluctuations folded in. The first term is due to multiple scattering and errors in $B$ field determination [23], while the second term comes from chamber measurement errors. The momentum measurement provided an additional means for linking muons to upstream spectrometer tracks.

\subsubsection{Muon hodoscope}

The muon trigger required at least one hit from each scintillator wall. This trigger was put in coincidence with a delayed pretrigger from the upstream portion of the experiment. The material upstream of the final hodoscope corresponded to $\mathbf{3 0}$ hadronic interaction lengths and 293 radiation lengths (26 and 258 respectively in the first run), virtually assuring that the triggering particle was a muon with an energy above $6 \mathrm{GeV}$ (5 $\mathrm{GeV}$ for the first run).

The upstream scintillator wall was located $13.8 \mathrm{~m}$ downstream of the target and consisted of 36 horizontal counters arranged symmetrically about the vertical axis in two columns of 18 counters each, the total covering an active area of $3.6 \mathrm{~m}$ horizontal by $2.9 \mathrm{~m}$ vertical. The downstream wall consisted of $\mathbf{4 0}$ vertical counters of 1.7 $m$ in length arranged in two horizontal rows of 20 counters each, for a total active area of $3.5 \mathrm{~m}$ horizontally by $3 \mathrm{~m}$ vertically and was $15.2 \mathrm{~m}$ downstream of the target. Both timing and pulse height of the photomultiplier signals were recorded. The geometric acceptance for muon triggers was $230 \mathrm{mrad}$ horizontally and $200 \mathrm{mrad}$ vertically, which, together with momentum cuts, gave a computed trigger efficiency of $50 \%$ and $65 \%$ for centrally produced semi-muonic charm decays and semi-muonic beauty decays, respectively.

\subsubsection{Muon system performance}

The "internal" muon system efficiency for reconstructing tracks in the muon drift chambers and linking them across the toroid was $91.1 \%$. The linking efficiency between the muon system and the upstream spectrometer was $96.5 \%$ with the $3.5 \%$ lost in the Landau tail of the multiple scattering distribution, giving $87.9 \%$ efficiency for linking a muon with momentum $>8 \mathrm{GeV} / c$ to an existing upstream track. A plot of the momenta measured in the two spectrometer systems for such tracks is shown in fig. 25 . The upstream spectrometer efficiency for muon tracks was $93 \%$ with the losses due to software confusion in dense track regions. Thus, the overall muon finding and linking efficiency for prompt muons emerging from the target region into the geometrical acceptance of our detectors was $81.7 \%$. If these were the only muons present this would have been our 


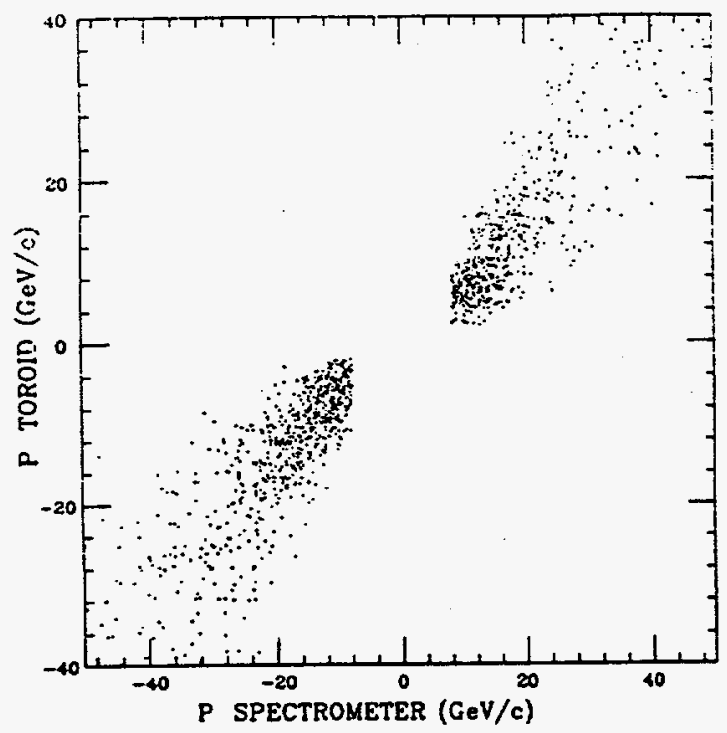

Fig. 25. Track momenta measured in muon spectrometer versus those measured in upstream spectrometer.

muon trigger efficiency. However, many muons were produced downstream of the target by meson decays in flight and by secondary interactions in LAC, $\mathrm{HadCal}$ and other parts of the apparatus. Except for meson acays with small decay angles, these muons were not anked to the upstream spectrometer and reduced the overall measured muon yield to $38 \%$ of all triggers.

\section{The trigger system}

The event trigger required an interacting beam particle and at least one penetrating muon. The beam was defined by a coincidence of three circular disk counters, the smallest $5 \mathrm{~mm}$ in diameter, in anticoincidence with the beam halo counters, which were square scintillators with appropriately-sized circular holes. A silicon wafer counter just upstream of the emulsion target vetoed pulse heights greater than three minimum ionizing particles to rule out upstream interactions. (This counter was not present in the first run.) The interaction counter was directly downstream of the emulsion and consisted of two $14 \mathrm{~mm}$ square silicon wafers each $0.3 \mathrm{~mm}$ thick and closely spaced along the beam. (fig. 26.) The interaction threshold was set at three minimum ionizing particles. In the first run a plastic scintillation counter having three times the nuclear interaction length of the wafers was used for this purpose.

A beam interaction coincidence together with the absence of a veto indicating that the data acquisition system was busy, constituted the pretrigger and gated the data acquisition modules. If no muon signal followed, the modules were fast-cleared and ready for the next trigger. If a muon signal arrived, digital conversion of the stored analog signals occurred, data were transferred to the computer and the event was recorded.

The muon signal consisted of a coincidence between the two scintillator walls already described. Table 5 shows the muon energy loss for the major components of the experiment. The event trigger required a muon of energy $6 \mathrm{GeV}$. In the first run $5 \mathrm{GeV}$ sufficed because there was less steel. Total triggers recorded were 5.4 and 9.8 million for the first and second runs respectively, corresponding to roughly 100 and 300 million interactions.

For calibration, test, and alignment purposes other trigger options could be programmed. For details see ref. [19].

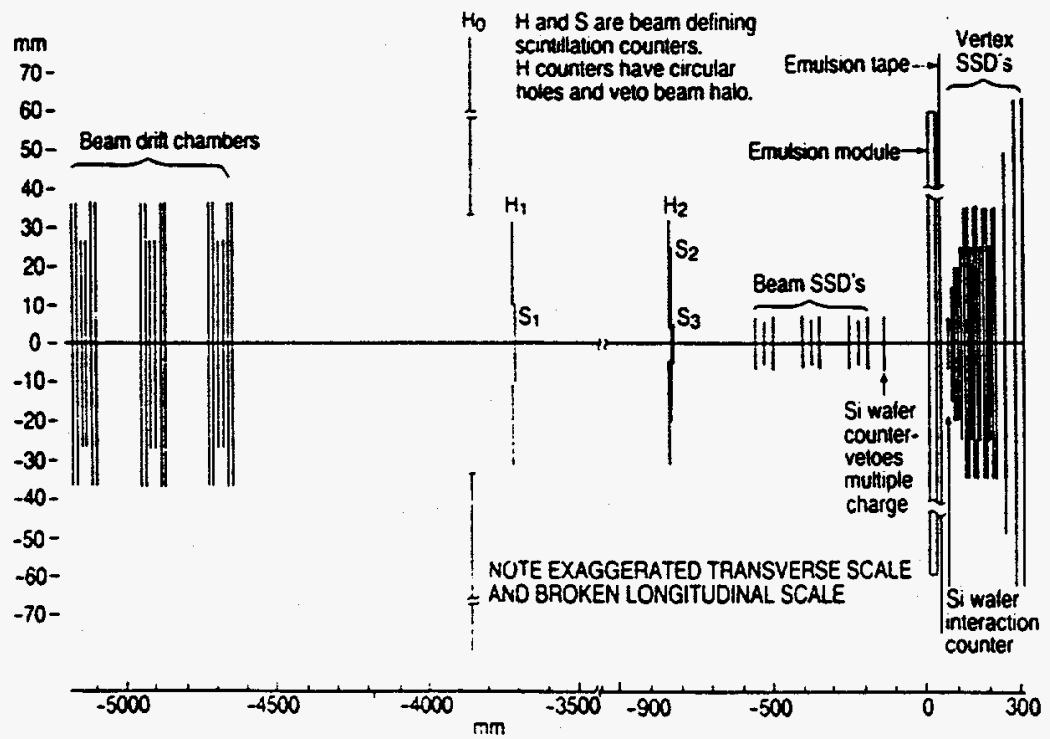

Fig. 26. Details of the upstream portion of the spectrometer including beam definition scintillation counters. 


\section{The data acquisition system}

The data acquisition (DAQ) system had to process hundreds of events per beam spill with each event averaging 27 kbyte of information. The beam came once a minute and the spill length was $10 \mathrm{~s}$ in the first run and $20 \mathrm{~s}$ in the second. The corresponding average number of events recorded per spill was 250 and 350 . For reasons to be discussed both Fastbus and Camac data acquisition modules (DAMS) were used and the data went to an LSI-11/73 front-end computer before being passed on to a VAX-11/750 host which wrote the tapes and handled most of the on-line processes.

\subsection{The data acquisition hardware}

With the exceptions mentioned below, all data acquisition modules in the experiment were commercially available. The system was basically Fastbus, but because 50 ps resolution Fastbus time-to-digital converters (TDCs) necessary for the time-of-flight and muon hodoscope system did not exist, LeCroy 2228 Camac TDCs had to be used. Target mover readouts from Nagoya, blind scalers, various monitoring devices, a "super trigger module" (STM) built at the Ohio State University and "smart" Camac crate controllers (SCCs) built at Fermilab completed the Camac portion of the DAQ system. The SCC eliminated a more complicated Camac data path required in the first run [19].

The Fastbus modules were all made by LeCroy and consisted of calibration and trigger modules, LeCroy 1810; ADCs, LeCroy 1885; TDCs, LeCroy 1879; Buffer Memory, LeCroy 1892 [20]; and segment manager/ interface (Crate Controller), LeCroy 1821. For full description of these devices see refs. $[19,21]$.

Events from the data acquisition modules were read into and preprocessed on a front-end LSI-11/73 computer running the RSX11-M operating system, prior to transmission to a VAX 750, which ran VMS system software and contained the major portion of the experiment's on-line system. The reason for using the LSI as the front end of the on-line system instead of basing it entirely on the VAX 750 was that no DR11-W driver interface for communication between a VAX and Fastbus 1821 controller existed, while an LSI driver for that purpose did.

\subsection{Overview of the data acquisition process}

Fig. 27 depicts the data flow in the front end of the experiment. The Fastbus data path is depicted with solid lines in the figure, while the Camac path is dashed. Upon receiving a trigger distributed by the Camac "super trigger module" (STM in the figure), analog pulses from the various components of the apparatus were read into Fastbus and Camac data acquisition

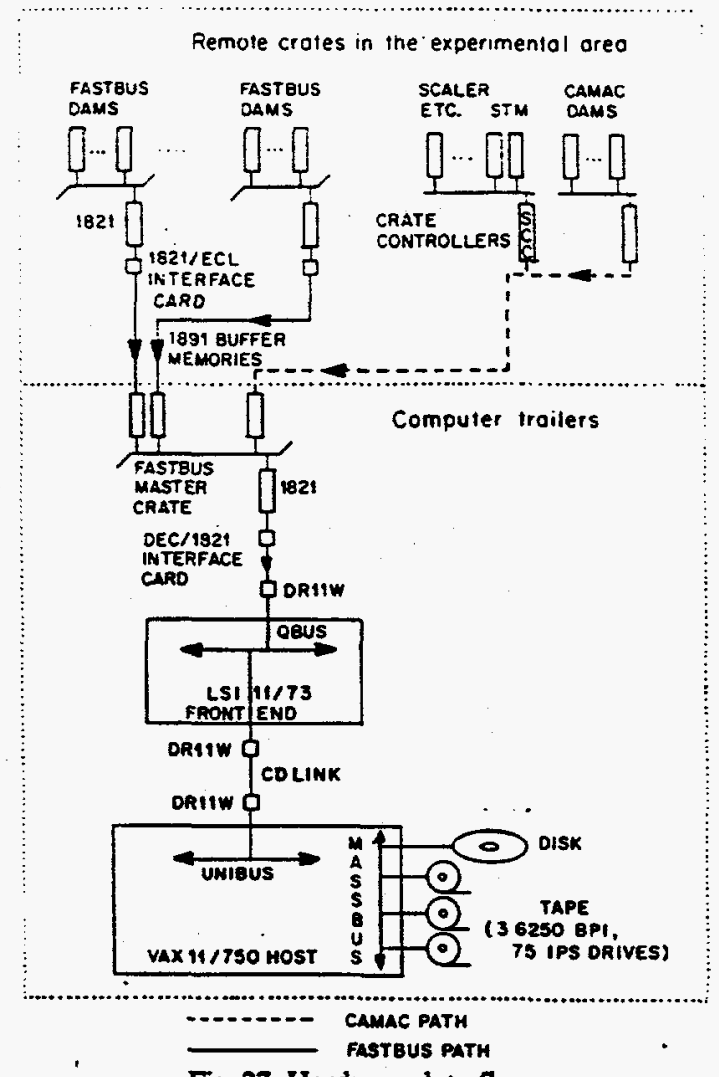

Fig. 27. Hardware data flow.

modules (DAMs). Signals from the SSDs, LAC, Had$\mathrm{Cal}$, and muon hodoscope were converted by the ADC modules into digital signals proportional to their integrated pulses. Discriminated time-over-threshold signals from the beam, spectrometer, and muon drift chambers, along with time information from the timeof-flight and muon hodoscope scintillation counters were converted by TDC modules into digital signals proportional to the time delay of the puise.

Due to the high readout speed of Fastbus compared to the reading speed of the LSI computer, the digitized data from the Fastbus DAMs were buffered in highspeed Fastbus memories (1 and 4 Mbyte LeCroy $1891 \mathrm{~s}$ and 1892s located in the "master" crate in fig. 27) during the data spill of $20 \mathrm{~s}$ ( $10 \mathrm{~s}$ in the first run), until they could be read into the LSI. This scheme required the Fastbus memories collectively to hold at least one spill's worth of data, and the ISI to empty the memories in one machine cycle.

\subsection{The on-line system}

The on-line system provided for final event assembly, detector monitoring, and logging the data to magnetic tape. Read-in routines brought the data into the LSI, which assembled the events and other routines 
transferred the events to the VAX. There, the events remained available to be accessed by different subprocesses which performed various tasks, such as writing to tape, monitoring individual detectors, accumulating hits and histograms. displaying graphics and scaler readings, and providing end-of-tape-summaries.

The system was rather complex [19] and capable of tagging and/or discarding events flawed by data transfer errors or memory overwrites. It had to perform its many tasks without conflict while assigning highest priority to data acquisition during beam spill. At each spill several hundred events were written to tape, with an average event size of 27 kbytes. Tape switch-over was automatic and occurred typically every $15 \mathrm{~min}$.

\section{Off-line analysis}

The task of the off-line analysis was to select a manageable number of interesting events for scanning in the emulsion targets with minimal loss of charm and beauty decays. This required a reduction factor of at least 100 in the raw triggers recorded.

Vertices were reconstructed using only well-measured VSSD tracks linked to spectrometer drift chamber tracks at the magnet midplane. In addition, since desired muon tracks would come from semi-muonic decay vertices, tagged muon tracks were not used in the construction of primary vertices. Both primary and secondary vertices were reconstructed, where a primary vertex was defined as the most upstream vertex formed with acceptable $\chi^{2}$ from three or more tracks including the beam. Various cuts were imposed to optimize the emulsion scanning efficiency for interesting events; $60 \%$ of all triggers had a primary vertex in the fiducial region of the target and $38 \%$ of these events had a fully reconstructed muon.

Typical criteria for charmed particle searches were a muon having momentum greater than $8 \mathrm{GeV} / c$, a transverse momentum with respect to the beam greater than $0.2 \mathrm{GeV} / c$, and an impact parameter at the primary interaction vertex longer than $50 \mu \mathrm{m}$. These cuts, plus the requirement of a vertex in the emulsion target, reduced the raw triggers by a factor of a hundred. Beauty selection criteria were more complex, and required correlated cuts on a number of variables which included the momentum. transverse momentum and impact parameter of trigger muons, and the presence of additional hadronic tracks not originating from the primary vertex.

Because of the large number of candidate events the emulsion analysis required the development of computer-aided microscope techniques, both automatic and semi-automatic, and especially designed for dealing with hybrid emulsion-spectrometer data [22]. In the emulsion target, decays of heavy quark particles were searched
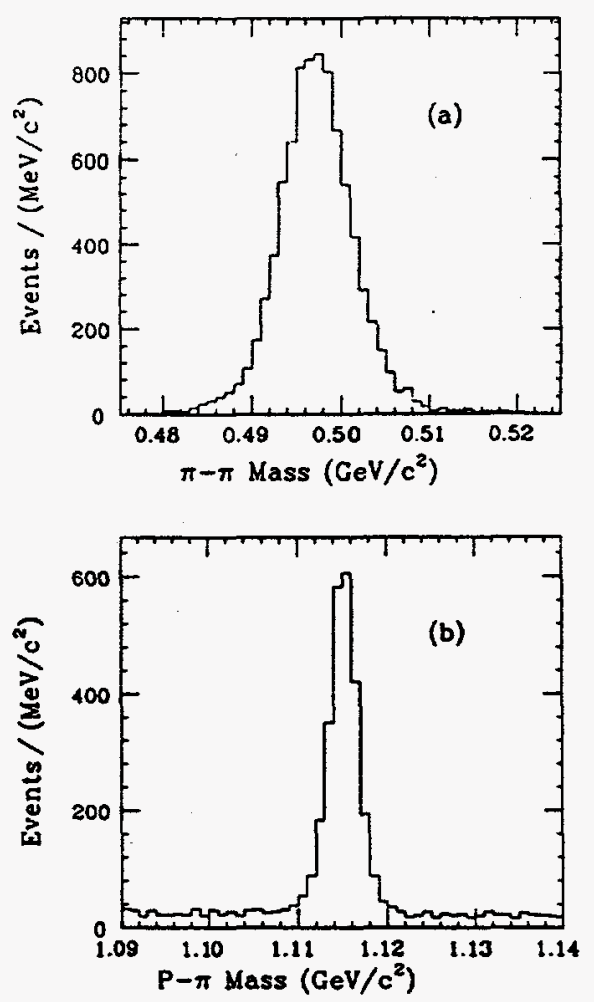

Fig. 28. Mass plot; (a) $\mathrm{K}_{s}^{0} \rightarrow \pi^{+} \pi^{-}$and (b) $\Lambda \rightarrow p \pi$.

for by following the spectrometer-selected muons up toward their origins [8]. Most of the remaining muons still originated from decays of pions produced in the primary interactions. In the emulsion analysis two thirds of these could be rejected very quickly by a computeraided graphic method of comparing the muon slopes with those of pions from the primary vertex. The remaining sample underwent the search procedure for charmed particle decays, with one out of seven events yielding a charm candidate.

The emulsion information was used to refine the track and vertex fits and momentum determinations in an iterative procedure. Secondary vertex tracks were combined to make kinematic fits to heavy quark particles. If the transverse momentum failed to balance, evidence for the predicted neutral particle was sought in the LAC and/or HadCal. To indicate the spectrometer resolution, fig. 28a shows the mass plot of a sample of $\mathrm{K}_{\mathrm{s}}^{0} \rightarrow \pi^{+} \pi^{-}$with reconstructed vertices in the region from the middle of the emulsion target to the second microstrip detector $(z=20-90 \mathrm{~mm})$. This distribution has a $\sigma$ of $4.0 \mathrm{MeV} / \mathrm{c}^{2}$ corresponding to a $1 \%$ mass resolution. Fig. $28 \mathrm{~b}$ shows the events consistent with $\Lambda \rightarrow p \pi$. Vertex reconstruction capability is illustrated in fig. 29, which shows, for a sample of the data, the longitudinal distribution of reconstructed primary vertices in the vertical emulsion target and adjacent 


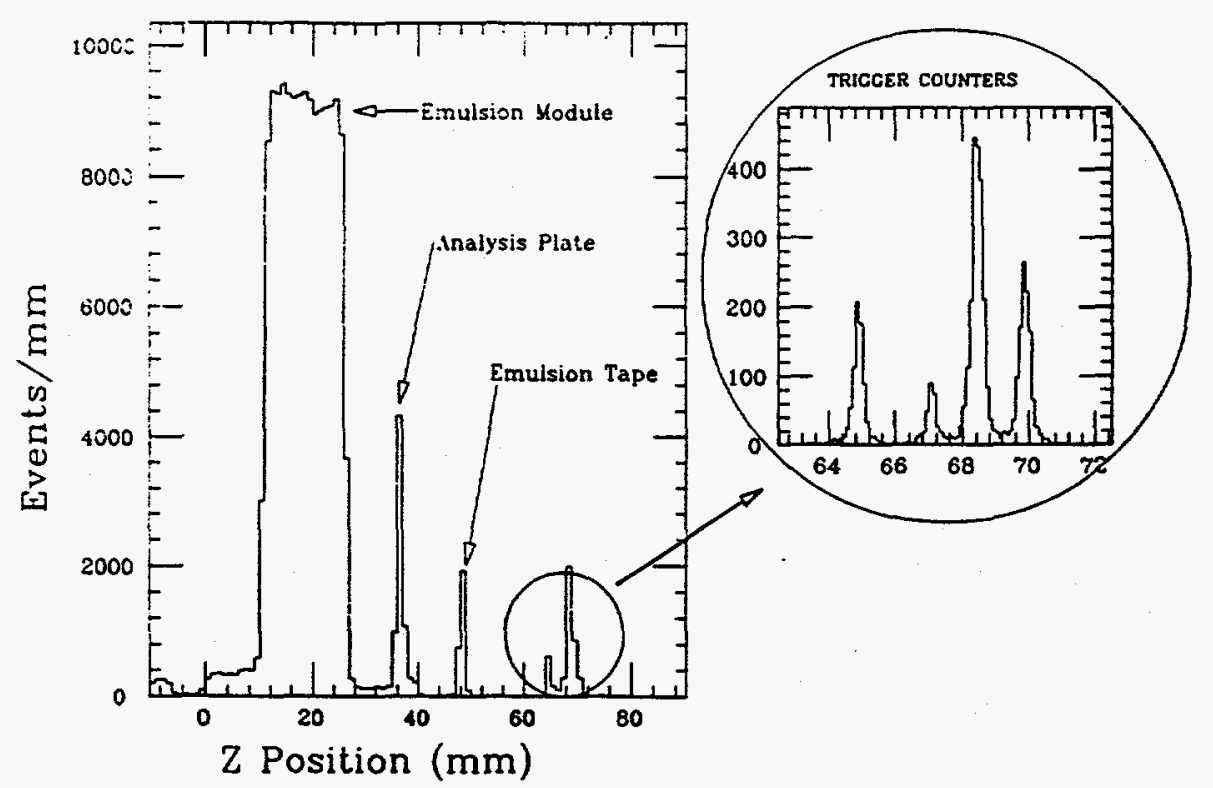

Fig. 29. Longitudinal distribution of reconstructed vertices.

portions of the apparatus. The measured $\sigma$ depended on the extrapolation distance from the first silicon detectors and was $270 \mu \mathrm{m}$ at the middle of the emulsion target, and less than $120 \mu \mathrm{m}$ for interactions in the trigger counter near the detector.

The mean number of reconstructed tracks from interactions in the emulsion was 14.7 (12.6 in the first run). The raw track finding efficiency as a function of angle is shown in fig. 30 , which displays, as crosses with error bars, the ratio of spectrometer tracks which are matched in slope and origin to tracks observed in the emulsion, to the number of tracks seen in the emulsion. Before corrections for unseen interactions in downstream material (silicon detectors, etc.), the track-finding ratio in the spectrometer was $84.9 \%$ for tracks with

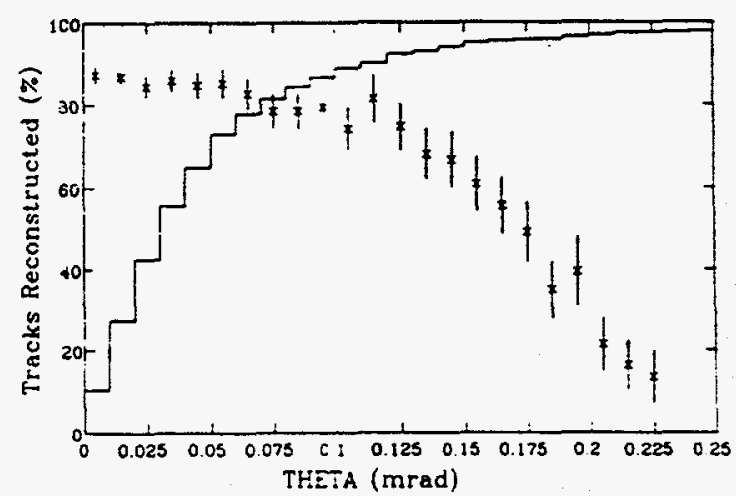

Fig. 30. Uncorrected track finding efficiency plotted versus angle (crosses with error bars); the solid curve is the integral angular snectrum for all tracks from muitiprong charm decay candidates observed in the emulsion. emission angle $\leq 0.100 \mathrm{rad}$. The falloff in track finding at large angles was due to the limited aperture of the silicon spectrometer and to tracks being swept out of the downstream drift chambers by the magnetic field. After accounting for these effects, the corrected efficiency was 91\%. The integral angular spectrum for tracks from multi-prong charm decay candidates is shown in the figure by the solid curve. It can be seen that the acceptance of the spectrometer was well matched to the physics.

\section{Summary}

A system designed to select heavy quark particles from the dense high multiplicity charged particle environment of fixed target hadronic interactions was described. It consisted of a sophisticated movable emulsion target followed by two spectrometers and included some particle identification. The upstream spectrometer contained high-resolution silicon microstrip detectors and vector drift chambers. These precise tracking detectors with their good double track resolution together with the special devices for the emulsion analysis were essential for achieving the goals of this experiment. The downstream spectrometer analyzed the filtered muons and provided the event trigger.

\section{Acknowledgements}

This work was supported in part by the US Department of Energy; the US National Science Foundation; 
The Japan Society for the Promotion of Science; the Japan-US Cooperative Research Program for High Energy Physics; the Ministry of Education, Science and Culture of Japan; the Korea Science and Engineering Foundation; and the Basic Science Research Institute Program, Ministry of Education, Republic of Korea.

\section{References}

[1] N. Ushida et al., Nucl. Instr. and Meth. 224 (1984) 50.

[2] N. Ushida et al., Phys. Lett. B206 (1988) 375.

[3] The configuration shown is for the second run. The first run had less steel and lacked the downstream spectrometer drift chamber embedded in the steel; neither did it have the emulsion tape, nor the graded size VSSDs.

[4] Although possible in principle, on-line cuts were not implemented.

(5) E.L. Berger, QCD Hard Hadronic Processes, ed. B. Cox, NATO ASI Series B vol. 197 (Plenum, New York, 1988) p. 501.

[6] In the first run the vertical analyzing region had five plates.

[7] This was composed of a $9.5 \mathrm{~mm}$ thick lucite honeycomb sandwiched between two fiber-reinforced-plastic plates of $0.25 \mathrm{~mm}$ thickness each.

[8] K. Taruma, Ph.D. thesis, Kobe University (1989), in Japanese.
[9] Our average measured charge multiplicity for $800 \mathrm{GeV}$ protons on emulsion was. 19; the corresponding number for $600 \mathrm{GeV}$ negative pions was 16 . These numbers do not include the nuclear breakup tracks.

[10] In the first run all 18 detectors were of the $5 \mathrm{~cm}$ type.

[11] LeCroy HQV810s originally built to Ohio State specifications.

[12] J.M. Dunlea, Ph.D. thesis, Ohio State University (1987).

[13] SCM-104 (on loan to Fermilab from Argonne National Laboratory).

[14] R.L. Ford and W.R. Nelson, SLAC report 210 (1978).

[15] The background for events with kinematic fits involving a neutral pion was determined by combining decay vertex information from the event of interest with calorimeter data from other emulsion events. Kinematic fits were then attempted for this "event mixed" sample. The fraction of successful fits was taken to be the background for the event.

[16] T.S. Jaffery, M.S. thesis, University of Oklahoma (1988).

[17] The field was generated using the CERN POISSON program.

[18] A. Mokhtarani, Ph.D. Thesis, University of Califomia, Davis (1988).

[19] G.A. Oleynik. Ph.D. Thesis, Ohio State University (1987).

[20] The first run buffer memories were LeCroy $1891 \mathrm{s.}$

[21] LeCroy Nuclear Products Catalogue 1989.

[22] S. Aoki et al., Nucl. Tracks 12 (1-6) (1986) 249.

[23] In the first run this term was 0.19 instead of 0.31 because there was less upstream steel. 


\section{Measurement of the Relative Branching Fraction $\Gamma\left(D^{\circ} \rightarrow K \mu \nu\right) / \Gamma\left(D^{\circ} \rightarrow \mu X\right)$ \\ (Version 3.0 July 1, 1990)}

K. Kodama(1), N. Ushida(1), A. Mokhtarani(2),(a), V.S. Paolone(2), J.T. Volk ${ }^{(2),(a)}$, J.O. Wilcox ${ }^{(2)}$, P.M. Yager ${ }^{(2)}$, R.M. Edelstein ${ }^{(3)}$, A.P. Freyberger $^{(3)}$, D.B. Gibaut ${ }^{(3)}$, R.J. Lipton ${ }^{(3),(a)}$, W.R. Nichols(3), D.M. Potter $^{(3)}$, J.S. Russ ${ }^{(3)}$, Y. Zhang ${ }^{(3)}$, H.I. Jang ${ }^{(4)}$, J.Y. Kim ${ }^{(4)}$, M.Y. Pac ${ }^{(4)}$, B.R. Baller ${ }^{(5)}$, R.J. Stefanski( ${ }^{(5),(b)}$, K. Nakazawa ${ }^{(6)}$, S. Tasaka ${ }^{(6)}$, Y.S. Choi $^{(7)}$, K.H. Chung ${ }^{(7)}$, D.C. Kim ${ }^{(7)}$, I.G. Park ${ }^{(7)}$, J.S. Song( ${ }^{(7)}$, C.S. Yoon ${ }^{(7)}$, M. Chikawa ${ }^{(\theta)}$, T. Abe ${ }^{(9)}$, T. Fujiji ${ }^{(9)}$, G. Fujioka ${ }^{(9)}$, K. Fujiwara ${ }^{(\theta)}$, H. Fukushima $^{\left({ }^{()}\right.}$, T. Hara ${ }^{(9)}$, Y. Takahashi ${ }^{(9)}$, K. Taruma ${ }^{\left({ }^{()}\right.}$, Y. Tsuzuki ${ }^{(9)}$, C.Yokoyama $^{(9)}$, S.D. Chang ${ }^{(10)}$, B.G. Cheon ${ }^{(10)}$, J.H. Gho ${ }^{(10)}$, J.S. Kang ${ }^{(10)}$, C.O. Kim${ }^{(10)}$, K.Y. Kim ${ }^{(10)}$, T.Y. Kim ${ }^{(10)}$, J.C. Lee(10), S.B. Lee ${ }^{(10)}$, G.Y. $\operatorname{Lim}^{(10)}$, I.T. $\operatorname{Lim}^{(10)}$, S.W. Nam ${ }^{(10)}$, T.S. Shin ${ }^{(10)}$, K.S. Sim ${ }^{(10)}$, J.K. Woo ${ }^{(10)}$, Y. Isokane ${ }^{(11)}$, Y. Tsuneoka ${ }^{(11)}$, S. Aoki ${ }^{(12)}$, A. Gauthier ${ }^{(12),(c)}$, K. Hoshino ${ }^{(12)}$, II. Jiitamura (12), M. Kobayashi(12), M. Miyanishi(12), K. Nakamura ${ }^{(12)}, M$. Nakamura ${ }^{(12)}$, Y. Nakamura ${ }^{(12)}$, S. Nakanishi ${ }^{(12)}, \mathrm{K} . \mathrm{Niu}^{(12)}, \mathrm{K} . \mathrm{Niwa}^{(12)}$, H. Tajima ${ }^{(12)}$, J.M. Dunlea ${ }^{(13),(d)}$, S.G. Frederiksen ${ }^{(13)}$, S. Kurainata ${ }^{(13),(e)}$, B.G. Lundberg(13),(a), G.A. Oleynik ${ }^{(13),(a)}$, N.W. Reay ${ }^{(13)}$, K. Reibel ${ }^{(13)}$, R.A. Sidwell(13), N.R. Stanion ${ }^{(13)}$, K. Moriyama ${ }^{(14)}$, H. Shibata(14), T.S. Jaffery ${ }^{(15),(a)}$, G.R. Kalbfleisch ${ }^{(15)}$, P.L. Skubic ${ }^{(15)}$, J.M. Snow ${ }^{(15)}$, S.E. Willis $^{(15),(f)}$, W.Y. Yuan ${ }^{(15)}$ O. Kusumoto ${ }^{(16),(g)}$, T. Okusawa ${ }^{(16)}, M$. Teranaka $^{(16)}$, T. Tominaga ${ }^{(16)}$, T. Watanabe ${ }^{(16)}$, J. Yamato ${ }^{(16)}$, H. Okabe ${ }^{(17)}$, J. Yokota ${ }^{(17)}$, M. Kazuno(18), F. Minakawa(18), E. Niu ${ }^{(18)}, \mathrm{S} . \mathrm{Ono}^{(18)}, \mathrm{H}$. Shibuya ${ }^{(18)}$, S. Watanabe ${ }^{(18)}$, Y. Sato ${ }^{(19)}$, I. Tezuka ${ }^{(19)}$, S.Y. Bahk ${ }^{(20)}$, and S.K. $\operatorname{Kim}^{(20)}$

\section{(Fermilab E653 Collaboration)}

(1) Aichi University of Education, Kariya 448, JAPAN

(2) University of California (Davis), Davis, CA 95616, USA 
(3) Carnegie-Mellon University, Pittsburgh, PA 15213, USA

(4) Chonnam National University, Kwangju 500-757, KOREA

(5) Fermi National Accelerator Laboratory, Batavia, IL 60510, USA

(6) Gifu Universily, Gifu 501-11, JAPAN

(7) Gyeongsang National University, Jinju 660-300, KOREA

(8) Kinki University, Higashi-Osaka 577, JAPAN

(9) Kobe University, Kobe 657, JAPAN

(10) Korea University, Seoul 136-701, KOREA

(11) Nagoya Institute of Technology, Nagoya $466, J A P A N$

(12) Nagoya University, Nagoya 464, JAPAN

(13) The Ohio State University, Columbus, OH $\$ 3210$, USA

(14) Okayama University, Okayama 700, JAPAN

(15) University of Oklahoma, Norman, OK 73019, USA

(16) Osaka City University, Osaka 558, JAPAN

(17) Science Education Institute of Osaka Prefecture, Osaka 558, JAPAN

(18) Toho University, Funabashi 274, JAPAN

(19) Utsunomiya University, Utsunomiya 350, JAPAN

(20) Wonkwang University, Iri 570-749, KOREA

(Received July 1, 1990)

The fraction $f$ of $D^{0}$ semimuonic decays which occur through the $K_{\mu \nu}$ mode has been measured in a hybrid enulsion spectrometer. Analysis of 141 semimuonic $D^{0}$ decay candidates gives $f=0.29 \pm 0.05$ (stat.) \pm 0.05 (syst.). From this measurement and existing data on the $D^{0}$ semileptonic branching ratio and lifetime, we obtain $R\left(D^{0} \rightarrow K^{-} \mu^{+} \nu\right)=(2.2 \pm 0.4 \pm 0.5) \%$ and $\Gamma\left(D^{0} \rightarrow K^{-} \mu^{+} \nu\right)=(5.2 \pm 0.9 \pm 1.1) \times 10^{10} \mathrm{sec}^{-1}$.

13.20.Fc, 14.40.Jz 
Semileptonic decays of charm are interesting because they appear to be relatively straightforward to calculate ${ }^{1,2,3,4,5,6}$. Annong the simplest such processes is the pseudoscalar decay ${ }^{7} D \rightarrow K l \nu$. Data on the partial rate for this decay has been used in some models ${ }^{1,3,4}$ to fix parameters for calculating otlier rates such as $D \rightarrow K^{*} l \nu$, which is expected to be roughly equal to that for $K \mu \nu$.

The $K l \nu$ rate has recently been measured in two ways: 1) by comparing ${ }^{8}$ the yield of $D^{0} \rightarrow K^{-} e^{+} \nu$ to that of $D^{0} \rightarrow K^{-} \pi^{+}$for photoproduced $D^{0}$ tagged by charged $D^{*}$ 's, and 2) by measuring ${ }^{9}$ the yield of $D^{0} \rightarrow K^{-} e^{+} \nu$ in a sample of tagged charm pairs produced in $e^{+} e^{-}$collisions. We present here the results of a complementary measurement, of the fraction $f$ of all $D^{0}$ semimuonic decays which occur through the $\mathrm{K}^{-} \mu^{+} \nu$ mode.

The data were taken in the first run of Fermilab Experiment E653, using a hybrid emulsion spectrometer described in detail elsewhere ${ }^{10,11}$. An $800 \mathrm{GeV}$ proton beam struck an emulsion target in which both primary interactions and decay candidates were observed. A charm-enriched sample of events to be scanned in the emulsion was selected by offline analysis of events recorded in the large-aperture spectrometer. Track reconstruction in 18 planes of silicon microstrip detectors downstream of the emulsion flagged events with possible decays. The spectrometer was triggered by interactions having muon candidates which penetrated $5 \mathrm{GeV}$ of range steel; this trigger enhanced the recorded muonic charm decays by an order of magnitude relative to hadronic decays. Final muon identification by the offline analysis required double momentum measurement: the candidate track had to be linked by tracking chambers through both a dipole spectrometer magnet and also an iron toroid which was part of the range steel.

A total of $5.4 \times 10^{6}$ triggers, corresponding to $1.0 \times 10^{8}$ interactions, was recorded in this proton run. Events to be scanned in the emulsion were selected on the basis of muon momentum $\left(p_{\mu}>8 \mathrm{GeV} / \mathrm{c}\right)$, muon transverse momentum $\left(p_{T \mu}>0.2 \mathrm{GeV} / \mathrm{c}\right)$, and evidence of a muonic secondary vertex from the silicon microstrip detectors. Primary vertices for such events were found in the emulsion with $94 \%$ efliciency.

Most recorded events were triggered by muons from $\pi$ and $K$ decay up- 
stream of the range steel. For those events in which the identified muon track did not match an emulsion track from the primary vertex, the muon track was projected back from the spectrometer to the downstream face of the emulsion, and if found there, was followed through the emulsion to a secondary vertex. Most multiprong secondary vertices in the emulsion were rejected as obvious interactions with dark nuclear breakup tracks or apparent nonconservation of charge. If an event was consistent with charm decay, spectrometer tracks were matched to those in the emulsion, and the emulsion was searched for other decay candidates.

The sample for the $D^{0}$ semimuonic decay study consists of $138 \mathrm{two}$-prong and three four-prong decay candidates with all emulsion tracks matched and momentum analyzed, and one track identified as a muon. These candidates survived careful scrutiny at high magnification for evidence of very short nuclear breakup lracks or Auger electrons. Because of the subinicron resolution and high grain density of the emulsion there is negligible chance of miscounting the number of prongs in the decays, or of failing to see the track of a charged parent. It was further required that no track have a transverse momentum $p_{\perp}$ relative to the charm direction of less than $0.25 \mathrm{GeV} / \mathrm{c}$; this eliminated all $K^{0}$ and $\Lambda^{0}$ decays.

Acceptances, reconstruction efficiencies and resolutions for the spectrometer were determined from a Monte Carlo simulation which generated events as hits in the spectrometer; these simulated events were then run through the complete chain of reconstruction and analysis programs. High energy interactions in emulsion were generated by the Lund Fritiof package ${ }^{13}$. Charm particles were superposed on these interactions as uncorrelated pairs with the distribulion in Feynman $x\left(x_{F}\right)$ and transverse momentum $p_{T}$ measured ${ }^{12}$ in this experiment: $\left(1-\left|x_{F}\right|\right)^{10} e^{-1.0 p_{T}^{2}}$.

There are two calegories of background in the two-prong sample: secondary vertices which are not charm decay (interactions of neutral hadrons), and charm decays which are not semimuonic (false muon identification). We have estimated the neutral interaction background from the measured number of two-prong secondary vertices which are flagged as interactions by nuclear breakup tracks, and which also contain an identified muon meeting the 


\section{PRELIMINARY}

above criteria: $14 \pm 6$ events. This number must be multiplied by the known fraction ${ }^{14,15,16}$ of low mulliplicity, high energy interactions in emulsion which have no evidence of nuclear breakup $(21 \pm .8 \%)$, and by $1 / 2$ for the requirement of apparent charge conservation, giving $1.5 \pm 0.9$ interactions in the two-prong sample of 138 decays. The estimated interaction background in the four-prong sample is comparable to the number (3) of decay candidates.

The contamination from two-prong hadronic decays of charm followed by $K^{ \pm}$and $\pi^{ \pm}$decay to tagged muons was determined by the Monte Carlo program to be $16 \pm 3 \%$. The small number of observed four-prong semimuonic candidates is good evidence that the feedihrough is not large, since for hadronic decays the four-prong rate is about $30 \%$ of that for two-prongs ${ }^{17}$.

The key quantity ${ }^{18}$ in determining the fraction $f$ of $K \mu \nu$ decays is the "minimum eflective mass" $M_{\min }$. This is the invariant mass of the $K \mu \nu$ system if the longitudinal momentum of the visible decay products in the parent resi frame can be ignored:

$$
M_{\min }=\sqrt{m_{v i s}^{2}+p_{\perp}^{2}}+\sqrt{m_{\nu}^{2}+p_{\perp}^{2}},
$$

where $m_{v i s}$ is the invariant mass of the detected charged particles from the decay (with a kaon mass assigned to the hadron), $p_{\perp}$ is the measured transverse momentum of the detected particles relative to the charm direction, and $m_{\nu}$ is the assumed mass of the missing neutral or neutrals, taken here to be zero. In this experiment the knowledge of $M_{\text {min }}$ is limited mainly by the measurement of the charm direction in the emulsion with a typical error of \pm 1.0 milliradian.

For true $K \mu \nu$ decays, $M_{\text {min }}$ has a prominent cusp at the $D^{0}$ mass (essentially a Jacobian peak effect); if the decay includes one or more neutral hadrons in addition to the neutrino, the $M_{\min }$ distribution has a broad maximum at lower mass. This is illustrated in Fig. 1, which shows Monte Carlo $M_{\min }$ distributions (including effects of spectrometer acceptance and resolution) for $D^{0} \rightarrow K^{-} \mu^{+} \nu$ (solid line), $D^{0} \rightarrow K^{*-} \mu^{+} \nu$ with $K^{*} \rightarrow K \pi$ (dashed line), and $D^{0} \rightarrow K^{*-} \pi^{0} \mu^{+} \nu$ (dotted line). The $K \mu \nu$ and $K^{*} \mu \nu$ Monte Carlo decays were generated with the matrix elements specified in Ref. 6.

The E653 spectrometer had no particle identification to distinguish between charged pions and kaons above $5 \mathrm{GeV} / \mathrm{c}$. Our analysis of both data 
and Monte Carlo decays always calculates $M_{\min }$ with a kaon mass assigned to the charged hadron. If this hadron is really a pion (as occurs for example in $\nu^{0} \rightarrow K^{*-} \mu^{+} \nu$, with $\left.K^{*-} \rightarrow \pi^{-} K^{0}\right)$, the $M_{\text {ruin }}$ distribution is shifted upward and has a tail extending above the $D^{0}$ mass. This effect is seen in the dashed and dotted histograms in Fig. 1, which include both the $K^{-} \pi^{0}$ $(33 \%)$ and $\pi^{-} K^{-0}(67 \%)$ decays of $K^{*-}$.

The fraction $f$ is determined as follows: 1) A maximum likelihood fit to the experimental distribution in $M_{\min }$ finds the single parameter $f_{\text {Raw }}$, which is the $K \mu \nu$ fraction in the experimental two-prong distribution:

$\ln \mathcal{C}=\sum_{\text {Events }} 0.84\left[f_{\text {Raw }}\left(I_{K \mu \nu}+0.11 I_{\pi \mu \nu}\right)+\left(1-1.11 f_{\text {Raw }}\right) I_{K \mu \nu X}\right]+0.16 I_{\text {Had }}$

where the $I_{i}\left(M_{\text {min }}\right)$ are Monte Carlo $M_{\min }$ distributions (normalized to unity) for $D^{0} \rightarrow K \mu \nu$, for $D^{0} \rightarrow \pi \mu \nu$ (fixed ${ }^{9}$ at $11 \%$ of $K \mu \nu$ ), for $D^{0} \rightarrow K \mu \nu X$, and for the $16 \%$ contamination from feedthrough of hadronic decays. 2) $f$ is then obtained from $f_{R a w}$ by correcting each contribution (including $1.5 \pm 1.5$ four-prongs) for experimental acceptance and efficiency.

The values of $f$ from the fits are not very sensitive to the choice of decay modes which comprise $I_{K \mu \nu X}$. We have tried a mixtures of the twoneutral mode $K^{*-} \mu^{+} \nu$ and the multineutral modes $K^{*-} \pi^{0} \mu^{+} \nu, \rho^{-} K^{0} \mu^{+} \nu$, and $\eta^{0} K^{-} \mu^{+} \nu$. The maximum number of two-prong decays in these multineutral modes is constrained by the observed number of four-prong candidates: $29 \%$ of $\eta^{0} K^{-} \mu^{+} \nu, 33 \%$ of $(\rho K)^{-} \mu^{+} \nu$ and $44 \%$ of $\left(K^{*} \pi\right)^{-} \mu^{+} \nu$ should be four-prong decays. We have not allowed fits which would contribute more than 3 observed four-prong events; this restricts the total two-prong multineutral branching ratio to be smaller than the $K^{*-} \mu^{+} \nu$ branching ratio in the most tolerant case.

Results for a fit using only the $K \mu \nu$ mode for $I_{K \mu \nu X}$, and for fits using the maximum allowed nultineulral contributions, are given in Table I. The $M_{\text {min }}$ distribution of the fit wilh best confidence level (C.L.) is compared with data in Fig. 2. Our final value of $f$ is determined by taking the average of the highest and lowest values of $f$ among the allowed fits. 


\section{PRELIMINARY}

TABLE I. $f_{\text {Raw }}$ and $f$ for Some Allowed $I_{K \mu \nu x}$.

\begin{tabular}{|c|c|c|c|}
\hline$I_{K \mu \nu X}$ & $f_{\text {Raw }}$ & $f$ & C.L. \\
\hline $\begin{array}{l}100 \% K^{*-} \mu^{+} \nu \\
80 \%\left(K^{*--} \mu^{+} \nu\right)\end{array}$ & $0.38 \pm 0.08$ & $0.29 \pm 0.06$ & $25 \%$ \\
\hline $\begin{array}{l}+20 \%\left(K^{*-} \pi^{0} \mu^{+} \nu\right) \\
50 \%\left(K^{*-} \mu^{+} \nu\right)\end{array}$ & $0.40 \pm 0.08$ & $0.29 \pm 0.06$ & $32 \%$ \\
\hline $\begin{array}{l}+50 \%\left(\rho^{-} K^{-10} \mu^{+} \nu\right) \\
60 \%\left(K^{*-} \mu^{+} \nu\right)\end{array}$ & $0.42 \pm 0.08$ & $0.25 \pm 0.05$ & $45 \%$ \\
\hline $\begin{array}{l}+40 \%\left(\eta^{0} K^{-} \mu^{+} \nu\right) \\
50 \%\left(K^{*-} \mu^{+} \nu\right) \\
+25 \%\left(\rho^{-} K^{0} \mu^{+} \nu\right)\end{array}$ & $0.46 \pm 0.08$ & $0.33 \pm 0.06$ & $28 \%$ \\
\hline$+25 \%\left(\eta^{0} K^{-} \mu^{+} \nu\right)$ & $0.46 \pm 0.08$ & $0.30 \pm 0.05$ & $49 \%$ \\
\hline
\end{tabular}

We determine $f=0.29 \pm 0.05$ (stat.) \pm 0.05 (syst.).

The systcmatic error includes contributions (combined in quadrature) arising from uncertainties in the inultineutral composition $( \pm 0.036)$, in $M_{\min }$ resolution $( \pm 0.015)$, in the number of real four-prong events $( \pm 0.015)$, in Monte Carlo statistics and parametrization $( \pm 0.015)$, in the treatment of backgrounds $( \pm 0.010)$, in the fraction of $\pi \mu \nu( \pm 0.015)$, and in the $x_{F}$ and $p_{T}$ dependence $( \pm 0.003)$.

An independent analysis ${ }^{19}$ of the same data was conducted using the distribution in transverse momentum of the muon from $D^{0}$ decay. The value of $f$ obtained is in good agreement with that from the $M_{\text {min }}$ analysis.

'The branching ratio and partial decay rate for $D^{0} \rightarrow K \mu \nu$ have been calculated by combining our value for $f$ with the world average values ${ }^{20}$ for the semileptonic branching ratio $R_{e}=R\left(D^{0} \rightarrow e^{+} X\right)=(7.7 \pm 1.1) \%$ and for the $D^{0}$ lifetime $\tau_{D^{0}}=(4.28 \pm 0.11) \times 10^{-13} \mathrm{sec}$; we take $R_{\mu}=R\left(D^{0} \rightarrow\right.$ $\left.\mu^{+} X\right)=0.96 R_{e}$. Then

$$
\begin{gathered}
R\left(D^{0} \rightarrow K \mu \nu\right)=(2.2 \pm 0.4 \pm 0.5) \% \\
\Gamma\left(D^{0} \rightarrow K \mu \nu\right)=(5.2 \pm 0.9 \pm 1.1) \times 10^{10} \mathrm{sec}^{-1} .
\end{gathered}
$$

This rate is smaller than the previousiy measured values ${ }^{8,8}$ of $(8.9 \pm 1.2 \pm$ 1.4) $\times 10^{10} \mathrm{sec}^{-1}$ and $(7.9 \pm 1.2 \pm 0.9) \times 10^{10} \mathrm{sec}^{-1}$. The weighted average 
of the three measurements, $7.1 \times 10^{10} \mathrm{sec}^{-1}$, has an acceptable chisquare probabability of $22 \%$.

The rate for $D^{+} \rightarrow K^{* 0} e^{+} \nu$ has been measured ${ }^{22}$ to be $(4.1 \pm 0.7 \pm 0.5) \times$ $10^{10} \mathrm{sec}^{-1}$, or $(23 \pm 5) \%$ of the $D^{+}$semielectronic rate ${ }^{20}$. Our $K \mu \nu$ result suggests that the discrepancy in $D^{0} \rightarrow K l \nu$ and $D^{+} \rightarrow K^{*} l \nu$ rales may not be as serious as previous resuits have indicated. If a smaller $K l \nu$ fraction is to be consistent with the $K^{* 0} l \nu$ rate ${ }^{22}$, there must be a significant branching ratio into other semileptonic modes.

We gratefully acknowledge the efforts of the Fermi National Accelerator Laboratory stafl in staging this experiment, and thank $R$. Morrison and $M$. Witherell for productive conversations. This work was supported in part by the US Department of Energy; the US National Science Foundation; the Japan Society for the Promotion of Science; the Japan-US Cooperative Research Program for High Energy Physics; the Ministry of Education, Science and Culture of Japan; the Korea Science and Engineering Foundation; and the Basic Science Research Institute Program, Ministry of Education, Republic of Korea.

a Presenl Address: Fermilab, Batavia, IL 60510.

${ }^{b}$ Present Address: SSC Laboratory, Dallas, TX 75237.

c Present Address: University of Illinois, Urbana-Champaign, IL 61801.

${ }^{d}$ Present Address: University of Rochester, Rochester, NY 14627.

- Present Address: Hirosaki University, llirosaki-city 036, JAPAN.

$f$ Present Address: Northern Illinois University, DeKalb, IL 60115.

g Present Address: Soai University, Osaka 559, JAPAN.

${ }^{1}$ M. Wirbel, B. Stech, and M. Bauer, Z. Phys. C29, 637 (1985).

${ }^{2}$ T. Altomari and L. Wolfenstein, Phys. Rev. D37 681 (1988). 
${ }^{3}$ M. Bauer and W. Wirbel, U. Heidellberg preprint HD-THEP 88-22 (1988).

${ }^{4}$ J.M. Cline, W.F. Palmer and G. Kramer, Phys. Rev. D40 793 (1989).

${ }^{5}$ D. Scora and N. Isgur, Phys. Rev. D40 1491 (1989).

${ }^{6}$ F.J. Gilman and R.L. Singleton, Stanford Linear Accelerator preprint SLAC-PUB-5065 (1989).

${ }^{7} D$ decays refer also to $\bar{D}$ decays throughout this paper.

8J.C. Anjos et al., Phys. Rev. Lett. 62, 1587 (1989).

${ }^{9} \mathrm{~J}$. Adler et al., Phys. Rev. Lett. 62, 1821 (1989).

${ }^{10} \mathrm{~K}$. Kodama et al., Nucl. Instr. \& Meth. A289, 146 (1990).

11J.M. Dunlea, Ph.D. Thesis, The Ohio State University (1987); G.A. Oleynik, Ph.D. Thesis, The Ohio State University (1987); K. Taruma, Ph.D. Thesis, Kobe University (1987) (in Japanese); T.S. Jaffery, M.S. Thesis, University of Oklahoma (1988); A. Mokhtarani, Ph.D. Thesis, University of California, Davis (1988); Y.L. Zhang, Ph.D. Thesis, CarnegieMellon University (1989); V.S. Paolone, Ph.D. Thesis, University of California (Davis) (1990).

${ }^{12}$ A.P. Freyberger, presentation at the XXV Rencontre de Moriond (1990); E653 Collaboration, to be published.

${ }^{13}$ B. Nilsson-Almquist et al., Comp. Phys. Comm. 43, 387 (1987).

${ }^{14} 11$. Winzeler, Nuc. Phys. 69, 661 (1964).

${ }^{15}$ S. IIasegawa et al., Suppl. Prog. Theo. Phys. 47, 126 (1971).

${ }^{16}$ We use the average of the values from Refs. 14,15 for three-prong interactions of $22.5 \mathrm{GeV}$ protons with no dark nuclear breakup tracks.

${ }^{17}$ M. Aguilar-Benitez et al., Z. Phys. C40, 321 (1988). The semileptonic decays (which are nearly all two-prong) have been subtracted.

${ }^{18} M_{\text {min }}$ is similar to the maximal missing mass used by $\mathrm{H}$. Palka et al., $\mathrm{Z}$. 
Phys. C35, 151 (1987) and by M. Aguillar-Benilez et al., Z. Phys. C30, 559 (1987).

${ }^{19} \mathrm{~S}$. Watanabe, Proceedings of the Japan-US Seminar "Present and Future of Experimental Particle Physics by Hybrid Emulsion - Electronic Detectors", Nagoya University (1990) p. 215.

${ }^{20}$ G.P.Yost et al. (Particle Data Group), Phys. Lett. B204, 1 (1988).

${ }^{21}$ D.M. Coffman, Ph.D. thesis, California Institute of Technology, 1986 (unpublished).

${ }^{22}$ J.C. Anjos et al., Phys. Rev. Lett. 62, 722 (1989).

FIG. 1. Monte Carlo histograms of experimental $M_{\text {nin }}$ distributions for the two-prong decays $D^{0} \rightarrow K^{-} \mu^{+} \nu$ (sulid), $D^{0} \rightarrow K^{*-} \mu^{+} \nu$ (dashed), and $\nu^{0} \rightarrow$ $K^{*-} \pi^{0} \mu^{+} \nu$ (dotted).

FIG. 2. Comparison of the experimental $M_{\min }$ distribution (error flags) with the best-fit sum of Monte Carlo distributions (daslied histogram). The Monte Carlo calculation for hadronic decay feedthrough (included in the solid histogram) is shown as a dotted histogram. 


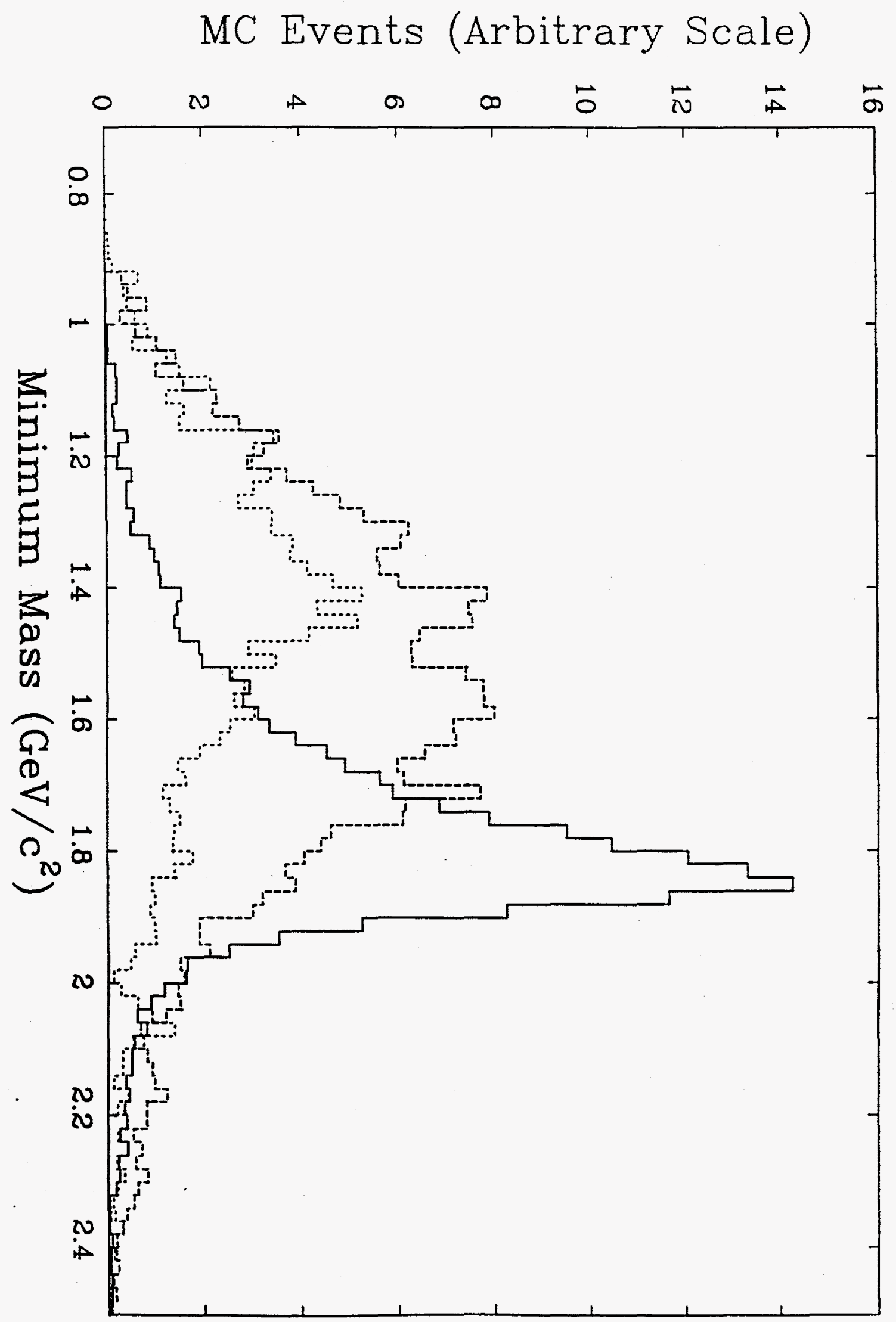

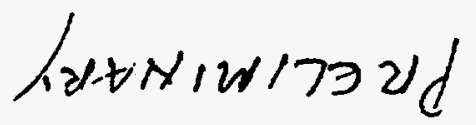




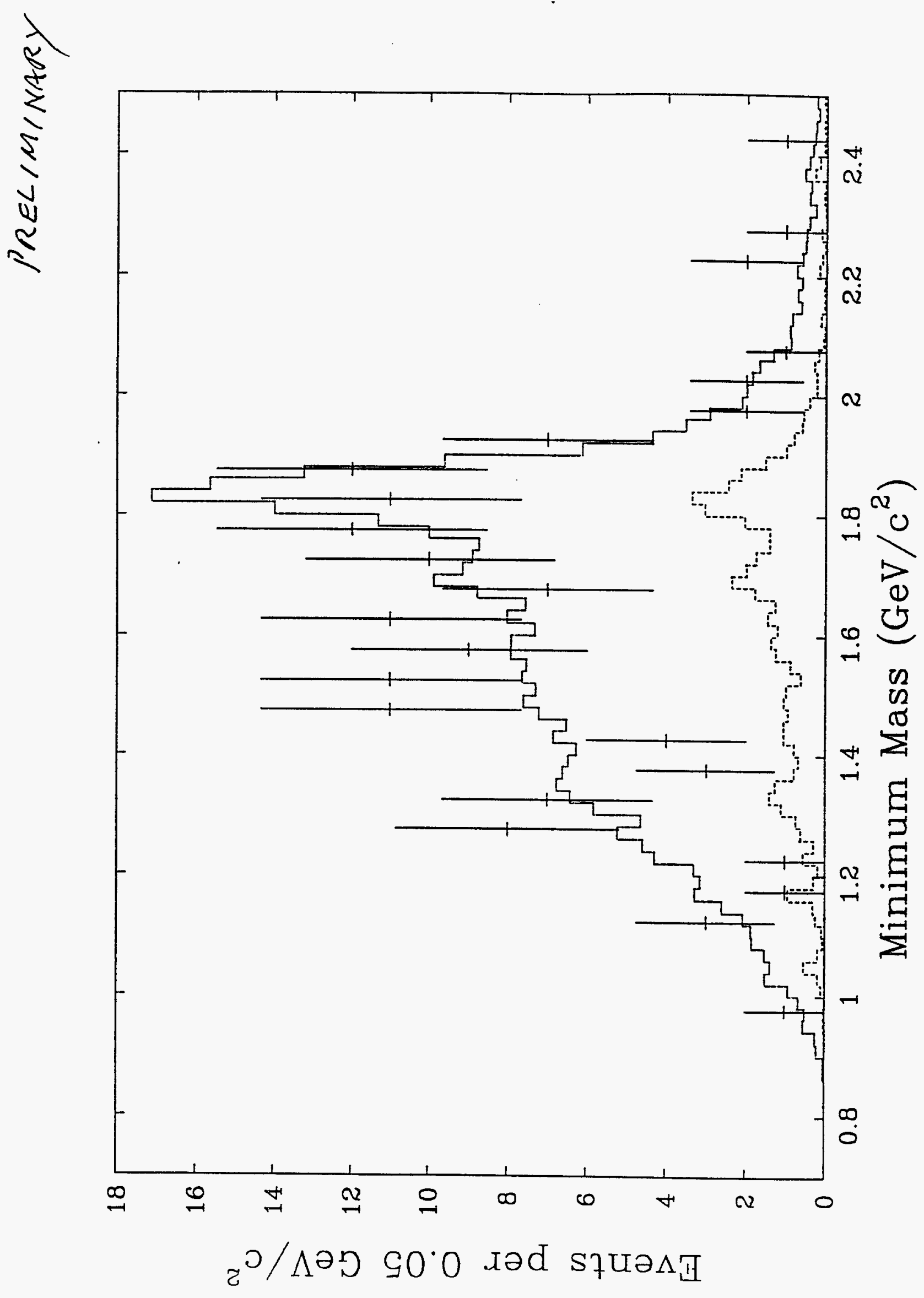




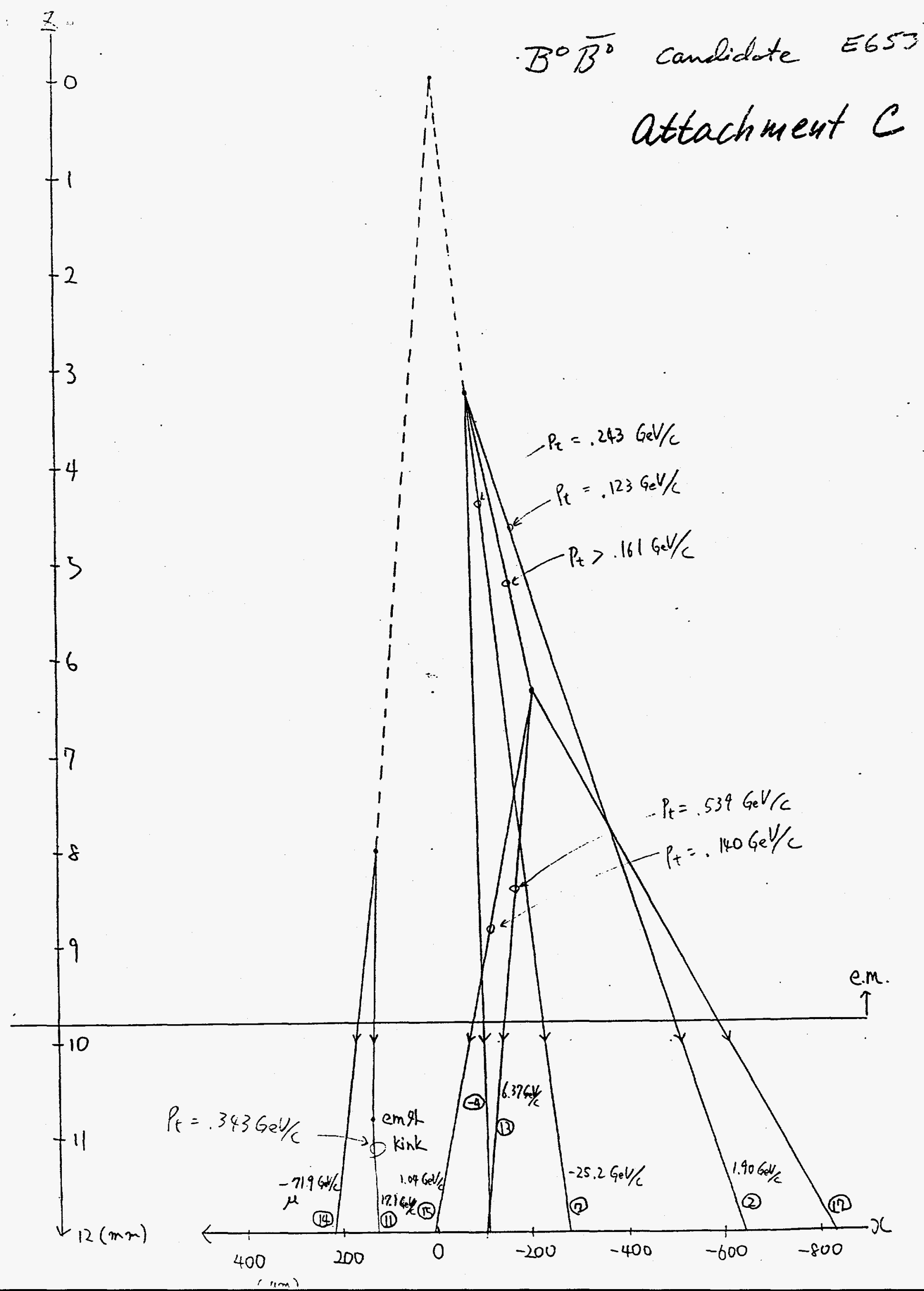




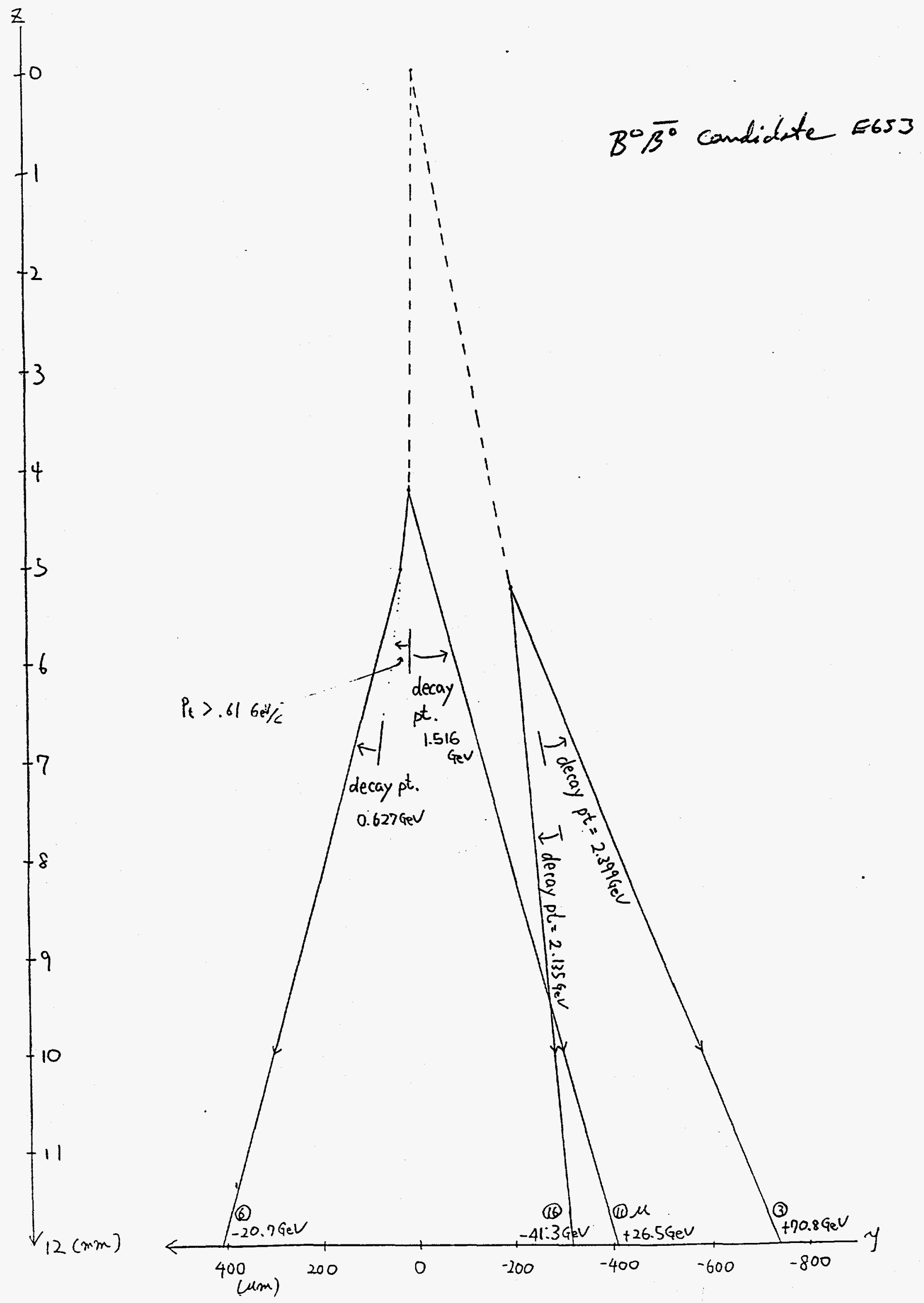




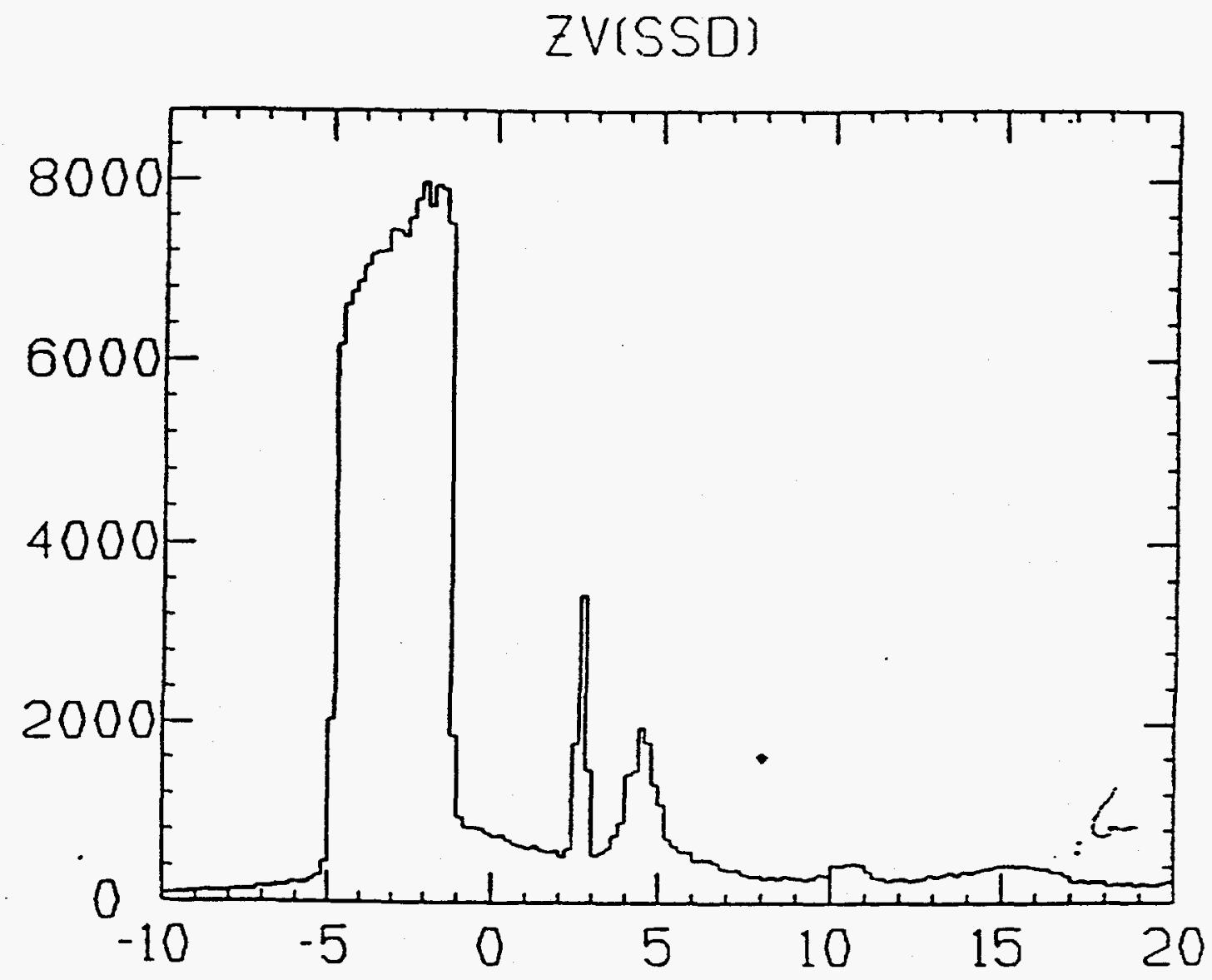




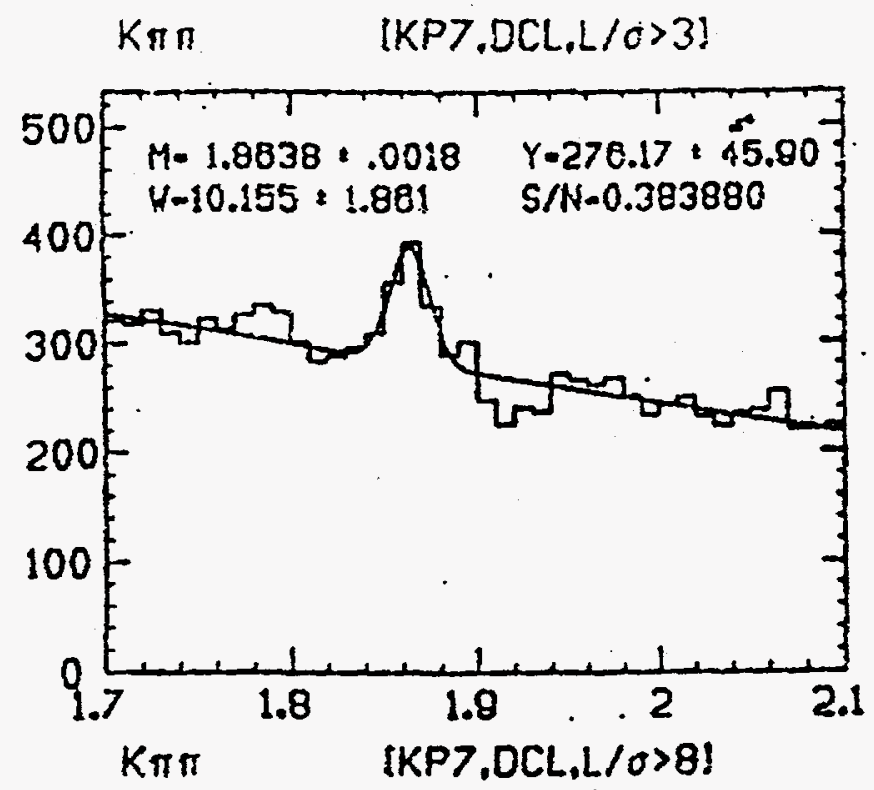

$\mathrm{K} \pi \pi$

[KPT.DCL.L/OO>5]
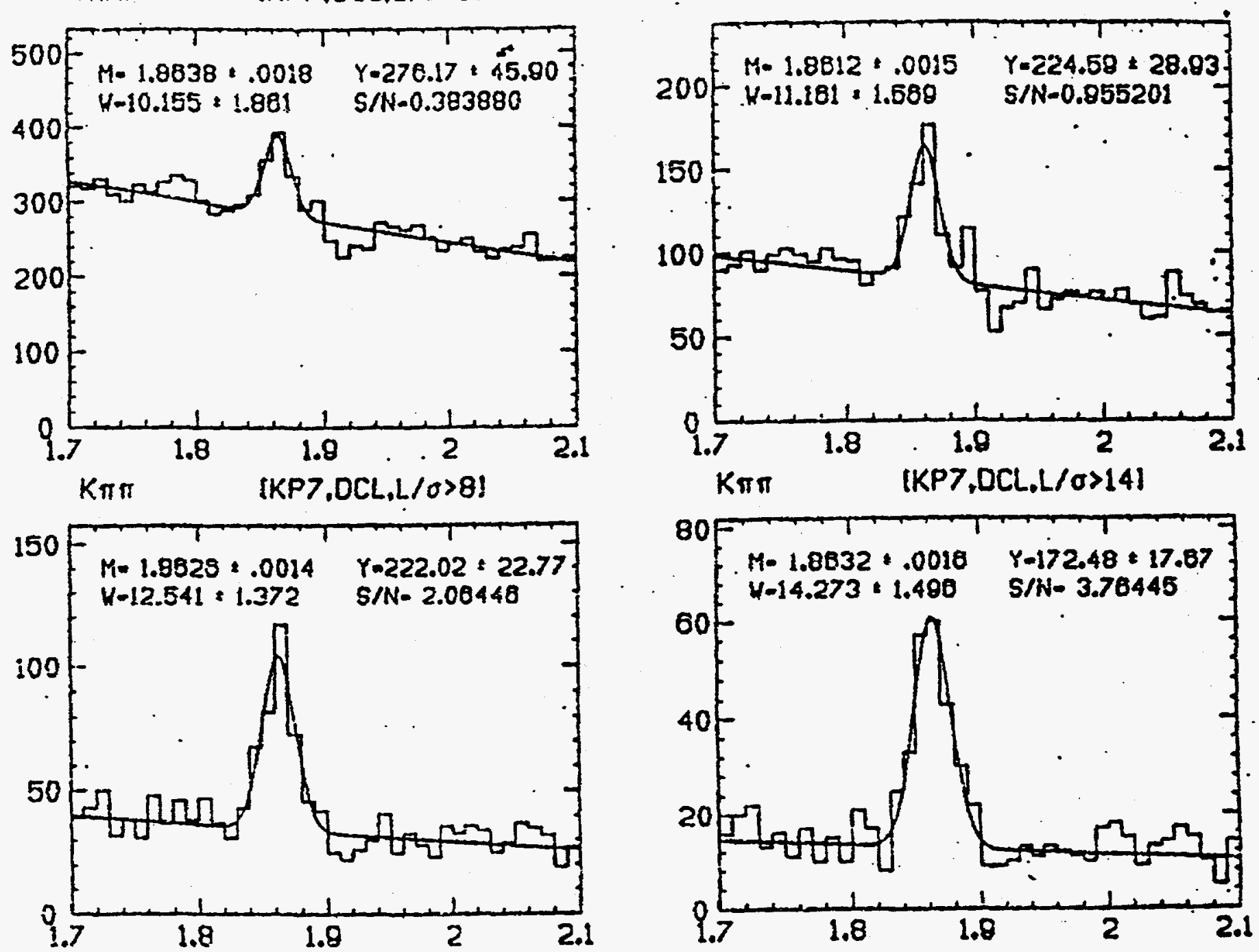
KS (CHI-SQUARED/DOF < 4)

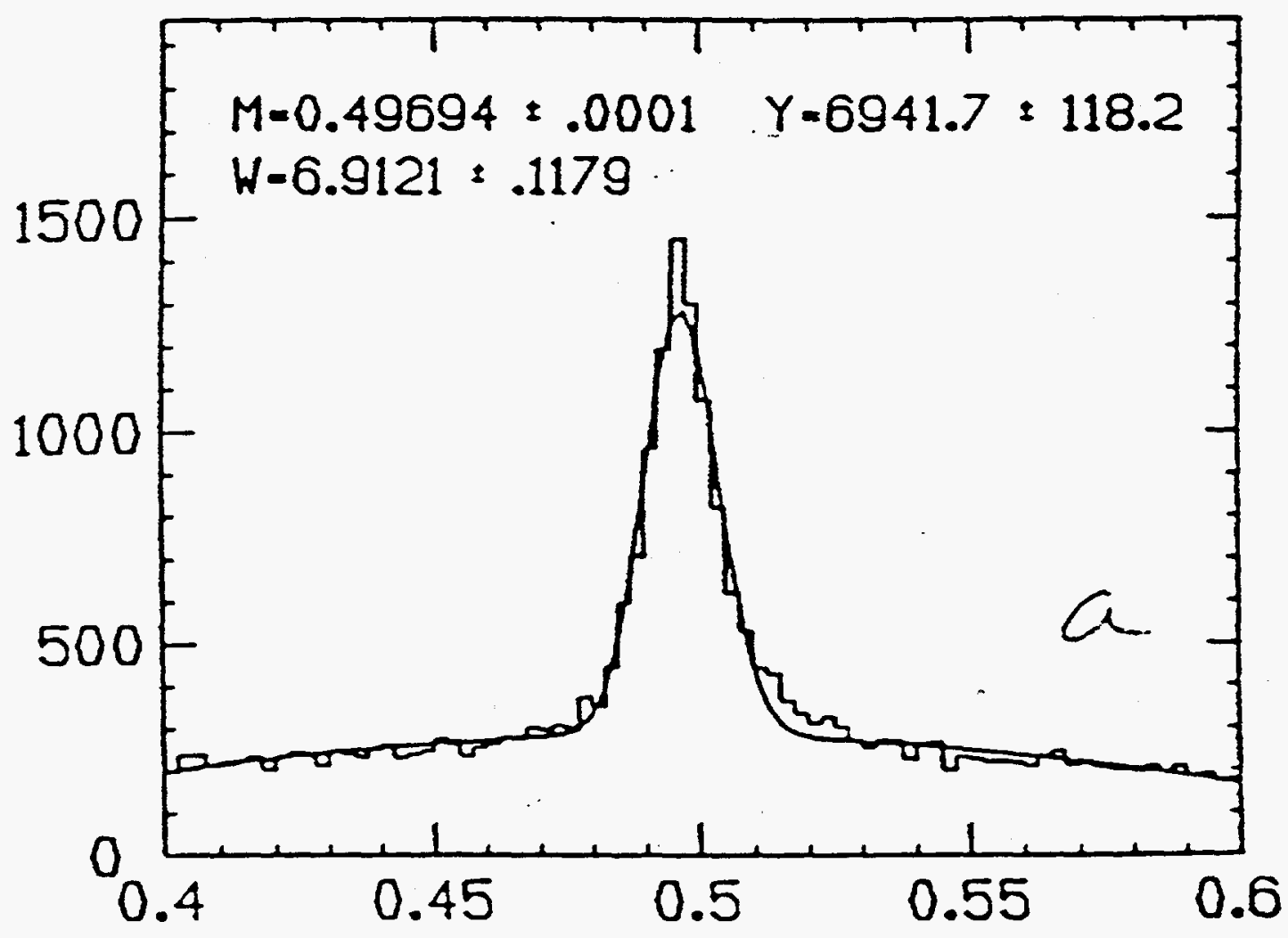




\section{Task F}

In 1990 , this task received an allotment of $\$ 10,000$ (plus an additional $\$ 3000$ allocated directly to SLAC) out of a request for approximately $\$ 85,000$ to participate in the TPC/Two Gamma experiment at high luminosity PEP. The experimental program for 1990 has been hampered by a number of problems: a serious earthquake, failure of equipment in PEP and in the SLC which acts as injector, and SLC program priorities. On the positive side, a former problem with excessive heating in the TPC vertex chamber has been solved and the switchover time between PEP injection and SLC running has been reduced to an acceptable value. We are hopeful that the severe problems have been overcome and that reliable high-luminosity running will commence in the summer of 1990.

Funding in the amount of $\$ 100,000$ was also received in 1990 for generic SSC detector R\&D on pixel devices. This has enabled us to continue our effort in this area, aimed at developing a vertex detector suitable for a general purpose tracking detector at the SSC.

\section{TPC/Two Gamma Experiment}

Modifications to the detector which have been completed for high luminosity running include the installation of a "straw" vertex chamber for B studies and the removal of nearly all of the equipment from the former Two Gamma experiment to accommodate the minibeta quads. The NaI tagger array remains, but in a new position much closer to the interaction point. The other remaining piece of apparatus, the forward drift chamber nearest the interaction point, was severely damaged by the earthquake and is being replaced by a chamber based on straws.

The modified apparatus was tested in a run during the Fall 1988 cycle during which PEP delivered $42.5 \mathrm{pb}^{-1}$. The most serious problem, overheating of the vertex chamber at high beam currents, has been corrected through the installation of a special cooling system. Rapid switching of the Linac beam has been achieved so that PEP filling can be interleaved with SLC running. 
- Pixel Detector Livelopment for the SSC

For the last two years, we have been involved in generic detector $R \& D$ on pixel detectors for SSC experiments. This is a collaborative effort with the advanced detector development group in the Physics Division at LBL, headed by D. Nygren. We are working in the following three areas:

- overall systems design and modeling,

- prototype pixel device tests and

- radiation damage testing.

\section{Systems Design}

We have participated in ongoing design studies of SSC vertex detectors based on "smart pixels" as parts of programs to develop (a) pixel devices for SSC experiments , (b) a silicon tracking subsystem and (c) a specific SSC detector emphasizing high pT physics and tracking .

On the basis of these efforts, test devices will soon be fabricated: a prototype p-i-n diode pixel array by LBL and prototype readout chips by Hughes Aircraft. These should be available for testing later this year. Design of the readout circuitry for these devices is being done by the UC Berkeley Space Sciences Laboratory (SSL).

We are developing a GEANT-based simulation of an SSC small radius pixel tracking chamber in collaboration with physicists in the Solenoidal Detector Collaboration (SDC) as part of a unified simulation of the entire detector.

\section{Prototype Tests}

We have set up a basic test facility using two CAMAC modules designed for the the Fermilab CDF silicon strip vertex detector and its associated VLSI readout chip (SVX). The SVX Readout Sequencer (SRS) generates arbitrary test pulse trains while the SVX Data Acquisition module (SDA) encodes and reads out analog responses using a flash $\mathrm{ADC}$.

We have developed a portable SVX data acquisition system based on a Mac- 
intosh computer with a National Instruments GPIB (IEEE-488) interface for use in tests at the Davis cyclotron and elsewhere. The CAMAC modules are accessed via a DSP Technology CC-488 GPIB/CAMAC crate controller. This allows us to use other remote GPIB equipment (programmable power supplies, DMMs, etc.) in our tests. Although GPIB is perhaps the most widely used interfacing standard for industrial test equipment, it is usually thought of in the context of "desktop-scale" experiments without requirements for high data transfer rates. One can, however, use a bus extender to control equipment $30 \mathrm{~m}$ away and still achieve data transfer rates in excess of 200,000 bytes/sec. This is more than adequate for our tests.

This system has been used to read out small ( $7 \mathrm{~mm}$ square, $50 \mu \mathrm{m}$ pitch) strip detectors produced at LBL using processes similar to those which would be applied in making p-i-n diode pixel arrays. The devices can be used for tracking in beam tests to study the performance of pixel arrays. In addition, the SRS/SVX and CAMAC system could in principle be used as a general purpose readout device for pixel array testing. Bench tests can be done using pulsed infrared-emitting LED's or radioactive sources. We are also investigating the use of a parallel, low intensity beam of $68 \mathrm{MeV}$ protons at the UCD cyclotron for initial beam tests.

\section{Radiation Tests}

We have acquired a Hewlett-Packard 4145B Semiconductor Parameter Analyzer and a probe station for detailed characterization of sample devices before and after irradiation. Additional instrumentation will be obtained this year.

We have performed an initial check of the "baseline" for comparing radiation damage done by $63 \mathrm{MeV}$ protons at the UCD cyclotron with that of higher energy protons. We exposed a single Hamamatsu S1723-06 p-i-n photodiode to a fluence of $0.76 \times 10^{12}$ protons $/ \mathrm{cm}^{2}$ over a period of $1 \mathrm{hr}$. The leakage current was subsequently measured daily over an interval of more than two months. We compared the leakage with preliminary results from Sadrozinsky et al. using similar diodes and $800 \mathrm{MeV}$ protons at Los Alamos. The flux was much lower in their test, but the fluence was approx. $10^{12}$ protons $/ \mathrm{cm}^{2}$.

The work of Summers et al. correlating damage in bipolar junction transis- 
tors with nonionizing energy deposition suggested that the damage factor would be higher by a ratio of 2.0 at the lower energy. The measured ratio was 1.9 , both after 29 days and after 58 days of annealing at room temperature. Our measurement is preliminary, based on a single sample, and subject to errors of order $10 \%$ or more, as is our interpretation of the results of Summers, et al.. But the excellent agreement is encouraging for our continuing radiation damage tests at the UCD cyclotron.

We will do more tests this summer, aimed at studying the effects of fluences in the range of $10^{13}$ to $10^{14}$ protons $/ \mathrm{cm}^{2}$. In this regime, evidence exists for changes in the bulk resistivity of the semiconductor as well as other effects which make charge collection difficult. It is important to understand these effects because the inner radii of a pixel vertex detector could receive such fluences during the lifetime of an experiment at the SSC. An abstract of a paper on these topics was submitted to the Symposium on Detector Research and Development for the Superconducting Supercollider to be held in Fort Worth on October 15-18, 1990. 
THEORETICAL TASKS

Task B - Theory, Particle Physics

Task E - Theory, Particle Physics 


\section{Publications, Preprints and Activities: Task B}

The publications and preprints produced by the members of the Task B theory effort are listed. In addition, the seminars given, organizational and adminstrative contributions, conferences and workshops participated in, and other major collaborative activities are outlined.

\section{J.F. Gunion: Publications/Preprints June 1, 1990-June 1, 1991}

1. J.F. Gunion, H.E. Haber, G. Kane and S. Dawson, "The Higgs Hunters Guide", Addison and Wesley (1990), Frontiers in Physics Series, second edition.

2. J.F. Gunion and D. Wyler, "Inducing a Large Neutron Electric Dipole Moment via a Quark Chromoelectric Dipole Moment", Phys. Lett. B248 (1990) 170.

3. J.F. Gunion and R. Vega, "The Electron Electric Dipole Moment for a $C P$-violating Neutral Higgs Sector", Phys. Lett. B251 (1990) 157.

4. J.F. Gunion, H.E. Haber, and J. Wudka, "Sum Rules for Higgs Bosons", Phys. Rev. D43 (1990) 904.

5. J.F. Gunion, R. Vega, and J. Wudka, "Naturalness Problems for $\rho=1$ and Other Large OneLoop Effects for a Standard Model Higgs Sector Containing Triplet Fields", Phys. Rev. D43 (1991) 2322.

6. D.A. Dicus, J.F. Gunion, and R. Vega, "Isolating the Scattering of Longitudinal $W^{+}$'s at the SSC Using Like-Sign Dileptons", Phys. Lett. B258 (1991) 475.

7. B. Grzadkowski, J.F. Gunion, and P. Krawczyk, "Neutral Current Flavor Changing Decays for the $Z$-boson and the Top Quark in Two-Higgs-Doublet Models", preprint UCD-90-34, to appear in Phys. Lett. B.

8. J.F. Gunion, "Associated Top-Antitop-Higgs Production as a Large Source of $W H$ Events: Implications for Higgs Detection in the Lepton-Neutrino-Photon-Photon Final State", Phys. Lett. B261 (1991) 510.

9. H. Baer, M. Drees, R. Godbole, J.F. Gunion and X. Tata, "Phenomenology of Light Top Squarks at the Fermilab Tevatron", preprint FSU-HEP-910308, to appear in Phys. Rev. D.

10. J.F. Gunion, "Non-minimal Higgs Sectors: Probes at 'Low-Energy' $e^{+} e^{-}$Colliders and in TopQuark Decays", to appear in Proceedings of the Workshop on Physics at Future Colliders, La Thuile 1990.

11. R.M. Barnett etal., including J.F. Gunion, "Report of the Subgroup on the Top Quark", preprint ANL-HEP-EP-90-98 (1990), to appear in Proceedings of the 1990 Summer Study on High Energy Physics, Snowmass, CO, June 25 - July 13, 1990.

12. J.F. Gunion, G.L. Kane, etal., "Overview of Progress in Higgs Boson Physics for the Superconducting Super Collider", preprint UCD-91-0010, to appear in Proceedings of the 1990 Summer Study on High Energy Physics, Snowmass, CO, June 25 - July 13, 1990.

13. J.F. Gunion and L. Roszkowski, "Ruling Out the Minimal Supersymmetric Model at LEP200", preprint UCD-90-26, to appear in Proceedings of the 1990 Summer Study on High Energy Physics, Snowmass, CO, June 25 - July 13, 1990.

14. J.F. Gunion and H.E. Haber, "Expected Limits on Supersymmetric Parameters at LEP-200", preprint UCD-90-27, to appear in Proceedings of the 1990 Summer Study on High Energy Physics, Snowmass, CO, June 25 - July 13, 1990.

$$
\text { B } 14 \text { (First Pg. of B) }
$$


15. R.M. Barnett, J.F. Gunion, and H.E. Haber, "Determining the Mass of the Gluino at the SSC", preprint UCD-90-28, to appear in Proceedings of the 1990 Summer Study on High Energy Physics, Snowmass, CO, June 25 - July 13, 1990.

16. J.F. Gunion and G. Kane, "Motivation for an SSC Detector with Ultrahigh Resolution Photon Detection", preprint UCD-90-29, to appear in Proceedings of the 1990 Summer Study on High Energy Physics, Snowmass, CO, June 25 - July 13, 1990.

17. J.F. Gunion, "Electric Dipole Moments for a $C P$-violating Neutral Higgs Sector", preprint UCD90-30, to appear in Proceedings of the 1990 Summer Study on High Energy Physics, Snowmass, CO, June 25 - July 13, 1990.

18. J.F. Gunion and H.E. Haber, "Higgs Boson Production in Photon-Photon Collisions at a High Energy $e^{+} e^{-}$Linear Collider", preprint SCIPP-90-22, to appear in Proceedings of the 1990 Summer Study on High Energy Physics, Snowmass, CO, June 25 - July 13, 1990.

19. R.M. Barnett, J.F. Gunion and B. Hubbard, "Determining the Mass of the Top Quark with the SDC Detector", preprint SDC-90-00146, to appear in Proceedings of the 1990 Summer Study on High Energy Physics, Snowmass, CO, June 25 - July 13, 1990.

20. R.M. Barnett, J.F. Gunion, H.E. Haber, I. Hinchliffe, B. Hubbard, and H. Trost, "Searching for Top Decays to Charged Higgs Bosons with the SDC Detector", preprint UCD-90-32, to appear in Proceedings of the 1990 Summer Study on High Energy Physics, Snowmass, CO, June $25-$ July $13,1990$.

21. G.H. Trilling etal., including J.F. Gunion, [Solenoid Detector Collaboration], "Letter of Intent by the Solenoidal Detector Collaboration to Construct and Operate a Detector at the Superconducting Super Collider", publication SSCL-SR-1153A.

22. J.F. Gunion, "An Overview of, and Selected Signatures for, Higgs Physics at Colliders", preprint UCD-91-9, to appear in Proceedings of the 'Workshop in High Energy Physics Phenomenology - II', Calcutta (January, 1991).

23. E. Ma etal., including J.F. Gunion, "Report From Working Group III: Quest for New Physics", to appear in Proceedings of the 'Workshop in High Energy Physics Phenomenology - II', Calcutta (January, 1991).

\section{J.F. Gunion: Invited Talks and Seminars June 1, 1990-June 1, 1991}

1. "Intermediate Mass Higgs Bosons: An Overview", Snowmass Workshop, June 27, 1990.

2. "Detecting $t \rightarrow H^{+}$Decays via a Violation of Lepton Universality", Snowmass Workshop, June $29,1990$.

3. "The Neutron Electric Dipole Moment", Snowmass Workshop, July 10, 1990.

4. "Large Contributions to the Neutron Electric Dipole Moment", Aspen Center for Physics, July 17,1990 .

5. "Electric Dipole Moments for a CP-violating Neutral Higgs Sector", SLAC, Sept. 5, 1991.

6. "Electric Dipole Moments for a $C P$-violating Neutral Higgs Sector", Institute for Theoretical Physics, Sept. 21, 1990.

7. "Electric Dipole Moments for a $C P$-violating Neutral Higgs Sector", Beyond the Standard Model - II, Oct. 31 - Nov. 3, 1990.

8. "Electric Dipole Moments for a CP-violating Neutral Higgs Sector", WHEPP-II Workshop, Jan. 1-14, 1991.

9. "Overview of Higgs Physics", WHEPP-II Workshop, Jan. 1-14, 1991. 
10. "Strongly Interacting Gauge Boson Theories - Heavy Higgs", WHEPP-II Workshop, Jan. 1-14, 1991.

11. "Electric Dipole Moments for a Neutral Higgs Sector", LBL, Jan. 28, 1991.

12. "Selected Signatures for Higgs Bosons: Standard Model and Beyond", SSC Physics Symposium, Feb. 25-27, 1991.

\section{J.F. Gunion: Meetings and Workshops June 1, 1990-June 1, 1991}

1. Workshop on Tests and Extensions of the Standard Model, Aspen Institute, July 2 - July 27, 1990.

2. 1990 Summer Study on High Energy Physics, Snowmass, CO, June 25 - July 13, 1990.

3. Beyond the Standard Model - II, Oklahoma U., Oct. 30 - Nov. 3, 1990.

4. Workshop in High Energy Physics Phenomenology - II, Calcutta, Jan. 1 - Jan 14, 1991.

5. SSC Physics Symposium, Madison, Wisconsin, Feb. 25 - Feb. 28, 1991.

6. SDC Detector Performance Workshop, SSC Laboratory, May 31 - June 1, 1991.

\section{J.F. Gunion: Additional Activities etc.., June 1, 1990-June 1, 1991}

1. Organizer, 'Intermediate Mass Higgs Bosons', Snowmass, 1990.

2. Organized 'Higgs Working Group', Workshop for High Energy Physics Phenomenology, Calcutta, India, Jan. 1 - Jan 14, 1991.

3. Member of Working Group on the Next Linear Collider, Stanford Linear Accelerator, Sept. 1990 - present.

4. Extensive organization of lobbying for the SSC within California. As I did last year, I again orchestrated the contacting of most of the California House and Senate members by individuals from their own districts, February-April, 1991. This year's effort does not seem to have been as successful as last year.

\section{J.E. Kiskis: Publications/Preprints June 1, 1990-June 1, 1991}

1. J. Kiskis, 'Behavior of Higher Representation Silson Lines in Finite-Temperature, $S U(2)$, Lattice Gauge Theory", Proceedings of the 1990 Meeting of the Division of Particles and Fields of the American Physical Society, Rice University, Editors B. Bonner and H. Miettinen, World Scientific, Singapore (1990).

2. J. Kiskis, R. Narayanan, and P. Vranas, "Random Walk, Critical Behavior, and Finite-Temperature" SU(2) Lattice Gauge Theory", Proceedings of the 1990 Meeting of the Division of Particles and Fields of the American Physical Society, Rice University, Editors B. Bonner and H. Miettinen, World Scientific, Singapore (1990).

\section{Orr: Publications/Preprints June 1, 1990-June 1, 1991}

1. Lynne H. Orr and W.J. Stirling, "The Longitudinal Structure Function at Small $x$ : the Effect of Next-to-Leading Order QCD Corrections and Heavy Flavor Thresholds", Phys. Rev. Lett. 66 (1991) 1673.

2. Lynne H. Orr, "Decay Versus Hadronization of Top Quarks Produced in Hadron Colliders", University of Chicago preprint EFI-90-78, Phys. Rev. D to be published. 


\section{Progress Report (J. Gunion)}

1. J.F. Gunion, H.E. Haber, G. Kane and S. Dawson, "The Higgs Hunters Guide", Addison and Wesley (1990) Frontiers in Physics Series, second edition.

The physics of Higgs bosons, whether that of the Standard Model or those of extended models, and their detection has become an extremely involved and extensive subject over the last few years. As a result, a group of us decided to write a Physics Report on the subject containing a systematic study and survey of all the accumulated knowledge, including a full detailing of existing experimental constraints on and future probes of the Higgs boson sectors of all attractive models considered in the literature to date. The material eventually reached such a length that we decided to turn it into a book. The first edition of the book has become something of a standard reference for this type of physics. It includes a complete listing of all relevant Feynman rules, branching ratios, and so forth. Also included are treatments of several areas where we felt old results could be improved or required correction. In addition, it contains much original material not available anywhere in the literature. The second edition, now in press, will update the first edition by including recent limits on Higgs bosons from LEP and top quark mass limits from CDF and elsewhere. A host of minor improvements and a few corrections have been incorporated.

2. J.F. Gunion and D. Wyler, "Inducing a Large Neutron Electric Dipole Moment via a Quark Chromoelectric Dipole Moment", Phys. Lett. B248 (1990) 170.

Recently, the possibility of developing a large neutron EDM in theories with $C P$ violation in the Higgs sector has been emphasized by Weinberg and others. Despite the large amount of attention this issue has received in the recent literature, we found a new contribution to the quark chromo-electric dipole moment that, in turn, yields a contribution to the neutron EDM which is larger than any of those previously considered in the literature. As part of this work, we also discuss in detail the (previously unappreciated) extent to which predictions for the neutron EDM are sensitive to the model employed to incorporate, at the hadron level, the relatively definitive quark/gluon level $C P$-violation predictions. The physics underlying the different models is explored.

3. J.F. Gunion and R. Vega, "The Electron Electric Dipole Moment for a $C P$-violating Neutral Higgs Sector", Phys. Lett. B251 (1990) 157.

Referring to the description of the previous project, $C P$-violating Higgs sectors also give rise to an EDM for the electron. An important top-loop contribution to the electron EDM was explored by Barr and Zee, along with an additional $W$-loop diagram that I pointed out to them. Nonetheless, there are many more diagrams, even within the Standard Model ('standard' aside from the Higgs sector) that had not been considered. We computed the contributions of the remaining diagrams, in particular those from an especially subtle and easily-overlooked class. Our results show that the contributions to the electron EDM from this latter sub-class are opposite in sign to, although somewhat smaller than, the $W$-loop diagrams computed by Barr and Zee. Since the top-loop contribution is also opposite in sign to the $W$-loop contribution, substantial cancellation occurs. As a result, it turns out that current experimental limits on the electron EDM are only just on the verge of providing significant constraints on the amount of explicit or spontaneous $C P$ violation that can be associated with the Higgs potential.

4. J.F. Gunion, H.E. Haber, and J. Wudka, "Sum Rules for Higgs Bosons", Phys. Rev. D43 (1990) 904.

The construction of consistent quantum field theories containing massive vector bosons presented a major challenge to theorists in the 1960's. In general, such models are non-renormalizable. Moreover, the tree-level amplitudes for scattering processes exhibit bad high energy behavior, i.e. they increase with the center-of-mass energy, in violation of unitarity. It was later shown that spontaneously broken gauge theories (with the possible addition of massive $U(1)$ vector bosons) are the only renormalizable and consistent quantum field theories involving massive vector boson states. Soon after, it was established that such theories are also the unique class of theories involving vector bosons in which the 
tree-level amplitudes for all scattering processes do not grow with the center of mass energy. ${ }^{[6]}$ Such a result is possible, because relations among parameters of the theory imposed by the gauge invariance are precisely what is needed to cancel out bad high energy behavior among tree-level Feynman diagrams. In other words, the required cancellation of bad high energy behavior leads to a series of sum rule relations among various coupling constants of the theory. These sum rules were examined more explicitly in refs. 8 and 9 , where bounds on Higgs boson masses were extracted. Nonetheless, the actual physical content of these sum rules remained somewhat obscure and difficult to extract from the existing literature. In this paper, we give a very explicit and easy to use formulation of the unitarity sum rules. In addition, we found a few discrepancies in some of the sum rules as previously published. We believe that we have have developed a presentation of the sum rules that is fully transparent to the experimental community. Our results will, we hope, soon become important since experimental accessibility to Higgs bosons is now becoming significant. Aside from the general formulation of the sum rules, we demonstrate their utility in a number of selected scenarios. When we discover one or more Higgs bosons, the most important immediate goal will be to measure their couplings and determine the extent to which they satisfy the unitarity sum rules. By doing so, we can determine whether the Higgs bosons and gauge bosons observed up to that moment could be a complete set, and if not the sum rules will provide a guide as to what Higgs bosons with what couplings remain to be discovered.

5. J.F. Gunion, R. Vega, and J. Wudka, "Naturalness Problems for $\rho=1$ and Other Large OneLoop Effects for a Standard Model Higgs Sector Containing Triplet Fields", Phys. Rev. D43 (1991) 2322.

Relatively little attention has been devoted to the subtleties associated with probing a Higgs sector that contains Higgs representations higher than doublets. In part this is because Higgs sectors with triplets and higher representations must be rather carefully constructed in order to avoid conflicting with the observed value of $\rho \equiv m_{W} /\left(m_{Z} c_{W}\right) \simeq 1$. Nonetheless, models can be easily constructed containing triplet Higgs representations (usually in combination with doublet representations in order to give fermions mass) which exhibit a custodial $S U(2)$ symmetry at tree-level that preserves $\rho=1$ even when the neutral members of the triplet representations acquire a non-zero vacuum expectation value. An example of such a model is that discussed by Georgi and collaborators. ${ }^{[10]}$ In a previous paper, we explored the rather intricate and subtle structure of the signatures for the Higgs bosons of this model. We found many unexpected signals, unusually long lifetimes, etc. for the Higgs bosons of the model. Production cross sections are also significantly dependent upon the amount of vacuum expectation value given to the triplet fields. All these issues were explored at some depth in the paper, and appropriate strategies for the detection of the Higgs bosons of such a triplet model outlined. Also presented were all the Feynman rules and couplings required for these and future calculations within this type of model, including the critical Higgs self-couplings.

The second paper is a continuation of our earlier work on Higgs triplet models. In particular, we have succeeded in demonstrating that even though $\rho=1$ can be made natural at tree-level as a result of imposing a custodial symmetry on the tree-level Higgs potential, the gauge interactions necessarily violate this custodial symmetry and lead to infinite contributions to $\rho$. In order to carry out the usual renormalization program for these infinite contributions, counterterms must be introduced into the Higgs potential that violate the custodial symmetry. Since only the infinite part of these counterterms is determined by the renormalization program, arbitrary finite violations of $\rho=1$ remain possible. While such violations can be related to other phenomenological observables, our conclusion is that in triplet models the value of $\rho$ must be counted among the observables required to fully specify the theory (in particular, the Higgs potential), and therefore $\rho$ is no longer predictable in such a model. We fully specify those observables related to the renormalization of $\rho$ that must be measured in order to test the internal consistency of this type of theory.

6. D.A. Dicus, J.F. Gunion, and R. Vega, "Isolating the Scattering of Longitudinal $W^{+'}$ 's at the SSC Using Like-Sign Dileptons", Phys. Lett. B258 (1991) 475. 
If the interaction of vector bosons $(V)$ becomes strong at high energy (as, for instance, in the Standard Model when the Higgs boson mass is taken to be large), then studying the interesting physics will require measuring $V V$ scattering processes $(q q \rightarrow q q V V)$ at subprocess energies at and above $1 \mathrm{TeV}$. A characteristic feature of such processes should be an enhanced probability for finding longitudinally polarized vector bosons in the final state. However, backgrounds to $V V$ dectection in final state decay modes containing quark jets from a $V$ decay are very large. Ideally one would like to study $V V$ final states in purely leptonic final state modes. Many channels for this can be envisioned $-W^{+} W^{-} \rightarrow l^{+} l^{-} \nu \bar{\nu}, Z Z \rightarrow l^{+} l^{-} l^{+} l^{-}$, and $W^{+} W^{+} \rightarrow l^{+} l^{+} \nu \nu$ (and charge conjugate). However, irreducible $q \bar{q}$ continuum electroweak backgrounds contribute to the first two final states, and are quite large. The $W^{+} W^{-}$channel also has a large background from $t \bar{t} \rightarrow W^{+} W^{-} b \bar{b}$ processes.

The $l^{+} l^{+}$channel has the advantage of having intrinsically much smaller backgrounds. The only irreducible background comes from $q q \rightarrow q q W^{+} W^{+}$production processes that yield transversely polarized $W^{+}$'s. The difficulty with this channel (as well as the $W^{+} W^{-}$channel) would seem to be determining when there is an enhancement in the number of longitudinaly polarized $W$ bosons in the final state. (One cannot detect the neutrino, so there is no chance of determining the $W^{+}$polarization directly.) In this study, we develop a number of cuts on the leptons, and an anti-tagging cut on the associated spectator jets that allow us to suppress the number of events in which the $W^{+}$bosons are produced with transverse polarization to a level below the number of events in which both $W^{+}$'s are produced with longitudinal polarization. In addition, we discuss how to combine these with a single jet tag cut in order to get rid of a remaining $t \bar{t}$ background coming from $t+\bar{t} \rightarrow l^{+} \nu b+j j \bar{c} l^{+} \nu$ in which the second $l^{+}$came from decay of the $\bar{b}$ emerging from the $\bar{t}$. The end result is that more than half of the interesting signal events (predicted for SM Higgs mass of $1 \mathrm{TeV}$ ) are retained, while the transverse polarization and $t \bar{t}$ backgrounds are reduced to less than $50 \%$ of the longitudinally polarized $W^{+} W^{+}$ signal. At canonical luminosity of $10 \mathrm{fb}^{-1}$ only a handful of signal events (about 8) remain, but for enhanced luminosity, detection of the longidutinally polarized $W^{+} W^{+}$signal should be possible. Further study of our techniques and alternative cuts is underway as reviewed later.

7. B. Grzadkowski, J.F. Gunion, and P. Krawczyk, "Neutral Current Flavor Changing Decays for the $Z$-boson and the Top Quark in Two-Higgs-Doublet Models", preprint UCD-90-34, to appear in Phys. Lett. B.

Two very interesting types of new physics are intimately connected — namely the physics of the top quark and the physics of a charged Higgs boson. For instance, a top quark can decay into a charged Higgs boson for $m_{t} \gtrsim m_{H^{+}}+m_{b}$, while the charged Higgs decay modes are dominated by $t \bar{b}$ if $m_{H+} \gtrless m_{t}+m_{b}$. Less obvious, but equally important, are the interconnections between a charged Higgs and the top quark in the area of rare $K$ and $B$ decays, and $\bar{K}-K$ and $\bar{B}-B$ mixing. For instance, in the latter, Feynman graphs involving the $H^{+}$are at least as important as the standard box diagrams involving the $W^{+}$. In an earlier paper, ${ }^{[11]}$ we combined the existing results for such mixing with limits from $b \rightarrow u$ decays on the crucial $V_{b u}$ entry of the Cabibbo-Kobayashi-Maskawa matrix to place limits, both on the top quark and on the two crucial parameters of a two-doublet Higgs sector - the mass of the $H^{+}$and the ratio of vacuum expectation values for the two Higgs doublets, normally called $\tan \beta$. Among other things we demonstrated that there is an $m_{t}$-dependent boundary in $m_{H^{+}}-\tan \beta$ parameter space that separates the domain of allowed from that of disallowed solutions. Roughly, the larger $\cot \beta$ is (i.e. the larger the $H^{+}$coupling to the $t \bar{b}$ ) the heavier the Higgs boson must be. These bounds are quite significant and will play an important role in suggesting the most reasonable ranges of parameter space in which to search for a charged Higgs boson. In the paper, we also demonstrate that the weak-mixing and $b \rightarrow u$ results combine (in the absence of a charged Higgs boson) to exclude a range of $m_{t}$ that depends upon the so-called bag parameters $B_{K}$ and $B_{B}$ describing the hadronic expectation value of the relevant quark-level matrix elements. Thus, discovery of the top quark at a particular mass, in combination with a fairly reliable lattice computation of $B_{K}$ and $B_{B}$ 
(something to be expected in the not too distant future) could easily imply that a charged Higgs boson (or other new physics that influences mixing) is required, and would place a strong constraint on its mass and the value of $\tan \beta$.

Charged Higgs bosons also can have a strong influence on flavor changing neutral current decays, such as $t \rightarrow c Z$. Such decays are predicted to have very small branching ratio in the SM without a charged Higgs. A charged Higgs boson will yield loop contributions in addition to those that involve the $W$ boson. In this paper, we investigated the magnitude expected for such FCNC decays given the constraints of the previous paragraph from $B$ and $K$ phenomenology. Although the charged Higgs can substantially enhance the rate of FCNC decays, one finds that the constraints from $B$ and $K$ phenomenology are such that the FCNC decays are still predicted to be too small for detection.

8. J.F. Gunion, "Associated Top-Antitop-Higgs Production as a Large Source of $W H$ Events: Implications for Higgs Detection in the Lepton-Neutrino-Photon-Photon Final State", Phys. Lett. B261 (1991) 510.

Hadron collider studies of past years have all suggested that detection of a SM Higgs boson in the intermediate mass range $\left(80 \leq m_{h_{S M}} \leq 2 m_{Z}\right)$ is something of a challenge. In earlier work, I and collaborators showed that $h_{S M} \rightarrow Z Z^{*} \rightarrow l^{+} l^{-} l^{+} l^{-}$decays provide a very clean signature for $130 \lesssim m_{h_{S M}} \lesssim 2 m_{Z}$. Masses below about $130 \mathrm{GeV}$ can in principle be probed by inclusive Higgs boson production followed by $h_{S M} \rightarrow \gamma \gamma$ decay. However, the large $\gamma \gamma$ and $\gamma j$ continuum backgrounds imply that very fine mass resolution in the $\gamma \gamma$ final state is required and that a very high level of $j e t-\gamma$ discrimination must be achieved. It is often suggested that a special purpose detector will be required. (For instance, the SDC detector will not have the necessary resolution and discrimination factor.)

Recently, a number of studies have pointed out that backgrounds in the $W+h_{S M} \rightarrow l \nu \gamma \gamma$ final state are much smaller, and that if there are enough signal events, detection of the Higgs boson should be possible even if the $\gamma \gamma$ resolution and $j-\gamma$ discrimination are not extremely good. The first production mechanism for such a final state that comes to mind is $q \bar{q} \rightarrow W^{*} \rightarrow W h_{S M}$. This was studied by a number of groups. ${ }^{[12]}$ However, the SSC rate predicted is very small for canonical $L=10 \mathrm{fb}^{-1}$ luminosity - roughly 4 to 5 events. Very recently, I and another group ${ }^{[13]}$ realized that the cross section for associated $t \bar{t} h_{S M}$ production, followed by one top quark decaying to and $W$ (which in turn decays to $l \nu$ ) and $h_{S M} \rightarrow \gamma \gamma$ leads to exactly the same background free $l \gamma \gamma$ final state. For canonical $L=10 \mathrm{fb}^{-1}$ luminosity, this process yields about 15 to 25 events after cuts throughout the mass range $80 \lesssim m_{h_{S M}} \lesssim 140 \mathrm{GeV}$. This development now allows us to say with confidence that the Superconducting Super Collider can detect the SM Higgs boson throughout the possibly crucial intermediate mass region using the combination of the $W h_{S M} \rightarrow l \nu \gamma \gamma$ and $Z Z^{*} \rightarrow 4 l$ final states.

9. H. Baer, M. Drees, R. Godbole, J.F. Gunion and X. Tata, "Phenomenology of Light Top Squarks at the Fermilab Tevatron", preprint FSU-HEP-910308, to appear in Phys. Rev. D.

It is, of course, widely known that the Fermilab CDF experiment can rule out squarks (if all are degenerate) and gluinos with masses below about $150 \mathrm{GeV}$, and a top quark with mass below about $89 \mathrm{GeV}$, provided that all decay canonically. In this paper, we considered the scenario, often encountered in a renormalization group treatment of supersymmetry, in which the stop squark ends up with mass substantially smaller than that of the other squarks, and even substantially smaller than the top quark itself. We demonstrate that this scenario leads to dramatically different phenomenology for top, squark, and gluino detection at Fermilab energies. In fact, it is not impossible that the top quark could be lighter than $89 \mathrm{GeV}$ and have remained undetected. A light stop squark is itself more difficult to detect since its cross section is only $1 / 6$ that normally employed in setting limits for six degenerate squarks, and, in addition, it has rather unusual decay modes. It is even possible that a light gluino (e.g. with mass of order $100 \mathrm{GeV}$ ) could be hidden by unexpected decays of the gluino to the stop squark. However, the Tevatron luminosity upgrade should go a long way towards eliminating (or confirming) this type of scenario. 
10. J.F. Gunion, "Non-minimal Higgs Sectors: Probes at 'Low-Energy' $e^{+} e^{-}$Colliders and in TopQuark Decays", to appear in Proceedings of the Workshop on Physics at Future Colliders, La. Thuile 1990.

In this talk, I reviewed the (then) available limits on Higgs bosons, in particular those appearing in models with non-standard Higgs sectors. Techniques for exploring such Higgs sectors at the Tristan and LEP $e^{+} e^{-}$colliders and at the Tevatron and CERN hadron colliders were detailed.

11. R.M. Barnett etal., including J.F. Gunion, "Report of the Subgroup on the Top Quark", preprint ANL-HEP-EP-90-98 (1990), to appear in Proceedings of the 1990 Summer Study on High Energy Physics, Snowmass, CO, June 25 - July 13, 1990.

This research report details the results of a number of subgroups in which I and others explored the physics of the top quark at existing and future accelerators. In particular, we developed techniques for accurate determination of the top quark mass in a variety of final state modes, and for detecting a charged Higgs boson should it appear in the decays of the top quark. The utility of polarization measurements was explored.

12. J.F. Gunion, G.L. Kane, etal., "Overview of Progress in Higgs Boson Physics for the Superconducting Super Collider", preprint UCD-91-0010, to appear in Proceedings of the 1990 Summer Study on High Energy Physics, Snowmass, CO, June 25 - July 13, 1990.

In this report, the results obtained by a number of subgroups exploring Higgs boson physics were amalgamated. Substantial progress was made at Snowmass in finding ways of probing Higgs boson scenarios that had previously seemed difficult to explore experimentally at hadron colliders such as the SSC. For instance, the $l \nu \gamma \gamma$ final state mode of associated $W+H i g g s$ production was given a first appraisal for the Standard Model. Various scenarios and appropriate detection techniques for the charged Higgs boson of a two-doublet model were explored, with particular attention to top quark induced backgrounds. The feasibility of detecting the presence of strong longitudinal gauge boson scattering using purely leptonic final states was demonstrated.

13. J.F. Gunion and L. Roszkowski, "Ruling Out the Minimal Supersymmetric Model at LEP200", preprint UCD-90-26, to appear in Proceedings of the 1990 Summer Study on High Energy Physics, Snowmass, CO, June 25 - July 13, 1990.

In this paper, we demonstrate that LEP-200 will be able to either confirm or rule out the Higgs sector predicted by the minimal supersymmetric model if the top quark mass is sufficiently small $(\lesssim 110 \mathrm{GeV}$ ) that radiative corrections to tree-level predictions are small. In particular, we focused on using $b$-tagging to isolate the signals from one or more of the neutral Higgs bosons of the model. We demonstrated that, with sufficiently efficient and pure $b$-tagging, separation of the Higgs signal from $Z Z$ continuum and other backgrounds should be feasible even in the most difficult case where the only kinematically accessible Higgs boson has mass near $m_{Z}$ and only relatively modest integrated luminosity is available.

14. J.F. Gunion and H.E. Haber, "Expected Limits on Supersymmetric Parameters at LEP-200", preprint UCD-90-27, to appear in Proceedings of the 1990 Summer Study on High Energy Physics, Snowmass, CO, June 25 - July 13, 1990.

In this report, we explore the region of minimal-supersymmetric-model parameter space over which detection of either neutralino-neutralino or chargino-chargino pair-production will be possible at LEP200. In particular, we demonstrate that chargino-chargino production will yield an observable rate essentially wherever such pair production is kinematically allowed. The portion of the supersymmetric $\mu-M$ parameter space that will thereby be explored is given.

15. R.M. Barnett, J.F. Gunion, and H.E. Haber, "Determining the Mass of the Gluino at the SSC", preprint UCD-90-28, to appear in Proceedings of the 1990 Summer Study on High Energy Physics, Snowmass, CO, June 25 - July 13, 1990. 
This paper develops a highly effective procedure for determining the mass of the gluino using the like-sign detection channel for gluino pair production. The particular mass variable and cuts employed are shown to provide a sensitive measure of the gluino mass at a hadron collider such as the SSC. Before including systematics due to model uncertainties, the error on the gluino mass determined in the suggested way can be as small as $5 \mathrm{GeV}$.

16. J.F. Gunion and G. Kane, "Motivation for an SSC Detector with Ultrahigh Resolution Photon Detection", preprint UCD-90-29, to appear in Proceedings of the 1990 Summer Study on High Energy Physics, Snowmass, CO, June 25 - July 13, 1990.

In this report, we review the many physics motivations for a detector capable of $\lesssim 1 \mathrm{GeV}$ resolution in $\gamma \gamma$ invariant mass. In particular, we focus on the sensitivity to new physics (such as new $W$ bosons or heavy fermion families) of the two-photon decays of Higgs bosons (both Standard Model and beyond). We argue that the potential for uncovering new high-mass physics, not to mention simply discovering one or more Higgs bosons, via the $\gamma \gamma$ spectrum is so great that a detector with ultra-fine resolution in this channel should be a priority.

17. J.F. Gunion, "Electric Dipole Moments for a $C P$-violating Neutral Higgs Sector", preprint UCD90-30, to appear in Proceedings of the 1990 Summer Study on High Energy Physics, Snowmass, CO, June 25 - July 13, 1990.

This survey reviews recent developments regarding contributions to electric dipole moments arising from a Higgs sector which exhibits spontaneous or explicit $C P$ violation. In particular, the work by Vega and myself on the electron's EDM and by D. Wyler and myself on the neutron's EDM was discussed and compared to other contributions and calculations. Sensitivity of the neutron EDM to techniques for turning quark/gluon level predictions into a prediction at the composite neutron level were outlined, as was the physics behind the various techniques.

18. J.F. Gunion and H.E. Haber, "Higgs Boson Production in Photon-Photon Collisions at as High Energy $e^{+} e^{-}$Linear Collider", preprint SCIPP-90-22, to appear in Proceedings of the 1990 Summer Study on High Energy Physics, Snowmass, CO, June 25 - July 13, 1990.

In this work, we demonstrate that production of neutral Higgs bosons (whether that of the Standard Model or those appearing in the Minimal Supersymmetric Model) via $\gamma \gamma$ collisions of back-scattered laser beams generally yields a very observable signal. For instance, we show that the Standard Model Higgs boson can be detected in either the $b \bar{b}$ or $Z Z$ decay modes (the mode depends on Higgs mass) for Higgs boson masses up to nearly the full $e^{+} e^{-}$machine energy of $\sqrt{s}=500 \mathrm{GeV}$ considered. This represents a significant improvement over the more conventional direct $e^{+} e^{-}$collision production of neutral Higgs bosons, which will only allow discovery of a SM Higgs boson up to masses of order $\frac{1}{2} \sqrt{s}$. Further, back-scattered photon-photon production allows detection of the SUSY Higgs bosons over much of the $\lesssim \sqrt{s}$ mass region, whereas conventional $e^{+} e^{-}$production at most will probe masses $\lesssim \frac{1}{2} \sqrt{s}$ and could have quite limited sensitivity to one or more of the SUSY neutral Higgs bosons.

19. R.M. Barnett, J.F. Gunion and B. Hubbard, "Determining the Mass of the Top Quark with the SDC Detector", preprint SDC-90-00146, to appear in Proceedings of the 1990 Summer Study on High Energy Physics, Snowmass, CO, June 25 - July 13, 1990.

This report details techniques that were developed by us at Snowmass for directly (i.e. via mass peak reconstruction) determining the top quark mass using $t \bar{t}$ pair production events at a hadron collider such as the SSC. The procedure we develop is at least as good as that based on the $e-\mu$ final state channel.

20. R.M. Barnett, J.F. Gunion, H.E. Haber, I. Hinchliffe, B. Hubbard, and H. Trost, "Searching for Top Decays to Charged Higgs Bosons with the SDC Detector", preprint UCD-90-32, to appear in Proceedings of the 1990 Summer Study on High Energy Physics, Snowmass, CO, June $25-$ July 13, 1990. 
This report presents the techniques that we developed for detecting a charged Higgs boson of a two-doublet Higgs sector when its mass is such that it is produced in the decays of the top quark. We show that two distinct procedures are adequate to ensure discovery of the $\mathrm{H}^{+}$over the entire physically interesting region of $\tan \beta$ space $(\tan \beta$ is the ratio of vacuum expectation values of the neutral doublet members). For small $\tan \beta$ we show how to detect the $H^{+}$via direct mass peak reconstruction in its hadronic decay modes (the dominant ones for small $\tan \beta$ ). When $\tan \beta$ is large, $H^{+}$decays of the top quark result in an easily detectable violation of $e-\mu-\tau$ universality. We find that the two detection techniques substantially overlap in $\tan \beta$ parameter space.

21. G.H. Trilling etal., including J.F. Gunion, [Solenoid Detector Collaboration], "Letter of Intent by the Solenoidal Detector Collaboration to Construct and Operate a Detector at the Superconducting Super Collider", publication SSCL-SR-1153A.

This is the principal document produced by the SDC collaboration as part of the detector approval process. My particular role in producing this document was in the area of physics processes. In particular, the PAC wished to have detailed results for a number of Higgs boson processes. An example is the detection of a charged Higgs boson in top quark decays (the project outlined just above). In fact, many of the Higgs detection techniques explored in the LOI had their origin in earlier work by myself and collaborators. My advice to and consultation with the experimentalists involved in SDC over the last five or so years played, I believe, a significant role in deciding upon the physics considerations used to determine the design of the detector.

22. J.F. Gunion, "An Overview of, and Selected Signatures for, Higgs Physics at Colliders", preprint UCD-91-9, to appear in Proceedings of the 'Workshop in High Energy Physics Phenomenology - II', Calcutta (January, 1991).

In this report, I give a wide-ranging review of Higgs physics, with particular attention to the most recently developed techniques for detecting Higgs bosons and/or strong $W$ scattering. Based upon the number of requests for this report that I have received, I would say that the community has found this summary of the current situation to be quite valuable.

23. E. Ma etal., including J.F. Gunion, "Report From Working Group III: Quest for New Physics", to appear in Proceedings of the 'Workshop in High Energy Physics Phenomenology - II', Calcutta (January, 1991).

In this report, we present results obtained at the WHEPP-II workshop by the New Physics working group. One of our focuses was the area of $C P$ violation in the Higgs boson sector, my principal area of interest at the workshop. We made significant progress in understanding the types of $C P$ violation that might arise, and what their physical manifestation at accelerators would be. Work in this area is still continuing. 


\section{Progress Report (L. Orr)}

1. Lynne H. Orr and W.J. Stirling, "The Longitudinal Structure Function at Small $x$ : the Effect of Next-to-Leading Order QCD Corrections and Heavy Flavor Thresholds", Phys. Rev. Lett. 66 (1991) 1673.

The behavior of the distribution of gluons inside the proton at small momentum fraction $x$ is of great interest to high energy physics for several reasons. Many processes at high energy hadron colliders such as the SSC are dominated by small $x$ gluons, and so it is necessary to know the gluon distribution in order to make accurate predictions for such experiments. There are now indications from QCD that the gluon distribution may be more singular at small momentum fraction $x$ than is usually assumed. Measurements of the longitudinal structure function $F_{L}$ at the $e p$ collider HERA will probe the gluon distribution function at smaller values of $x$ than has been possible in experiments up to now. These measurements should be able to distinguish between the standard and more singular behavior of the gluon distribution; however, this could be spoiled if higher order QCD corrections are too large. We studied the effects of next-to-leading order QCD corrections and heavy quark thresholds on predictions for $F_{L}$ at HERA with both standard and singular gluon distributions at low $x$. We found that the corrections are small (and in particular are smaller than the expected measurement uncertainties), so that the relationship between $F_{L}$ and the gluon distribution is preserved, and the standard and singular gluon distributions are still expected to be distinguishable at HERA. Thus we can remain confident that measurements at HERA will shed some light on QCD and the gluon distribution at small $x$. Our results have been published in Physical Review Letters. I continue to be interested in the subject of the gluon distribution, and I expect to become involved again as HERA experiments progress, through contact with experimentalists here at UC Davis who are members of the H1 collaboration.

2. Lynne H. Orr, "Decay versus Hadronization of Top Quarks Produced in Hadron Colliders", University of Chicago preprint EFI-90-78, Phys. Rev. D to be published.

Current limits place the top quark mass in the range where top's weak lifetime is comparable to typical time scales for hadronization. Thus a top quark, once produced, may decay before it has time to form a meson. In modeling top physics, whether for the top search at the Fermilab Tevatron or for experiments at the SSC where top provides enormous backgrounds to other physics of interest, one would like to know whether it is necessary to take hadronization into account. I addressed this question for top quarks produced in hadron colliders in an extension of previous work done in collaboration with J.L. Rosner. I compared the time scales for decay and hadronization for hadronically produced top quarks to determine for what top masses and momenta non-perturbative QCD effects are likely to appear before the top quark decays. I found that while top mesons are not necessarily expected to form, some hadronization effects can appear at the Tevatron up to masses of about $150 \mathrm{GeV}$; beyond that it is safe to neglect hadronization. My results for the SSC show that, because of the higher beam energies, hadronization effects can extend to higher masses; however, this may be modified substantially by higher order corrections. My results will be published in Phys. Rev. D. I am presently studying the perturbative side of the top fragmentation/decay interplay; see below. 


\title{
Progress Report (J. Kiskis and S. Staniford-Chen)
}

\author{
Introduction
}

The general goal of this research is to further the understanding of non-Abelian gauge fields in non-perturbative regions. We are particularly interested in the interplay of gauge symmetry, vacuum structure, and hadron structure.

Non-Abelian gauge theories are the foundation of the Standard Model. In particular, it is generally accepted that the $S U(3)$ gauge theory of quarks and gluons describes the strong interactions. For those physical processes that are controlled by short-distance interactions and that are relatively insensitive to large-distance effects, the results of perturbative calculations and experiments can be compared quantitatively. For most reactions, this is not the case because large-distance, nonperturbative effects are important. Confinement is only the most dramatic example; structure functions, hadronization, and strong interaction corrections to weak decays are important, practical issues.

The perturbative calculations that are valid at short distance where the effective coupling is weak require a gauge fixing that obscures to some considerable extent the fundamental role of gauge symmetry. Lattice gauge theory is an approximation that is complementary to continuum perturbation theory in that it maintains manifest gauge invariance while sacrificing Poincaré symmetry. The spacetime symmetry is restored as the correlation length for lattice fields diverges (as measured in lattice units) and the lattice spacing approaches zero (as measured in physical units). This occurs at a second order phase transition. The long-distance, nonperturbative effects are more accessible in the lattice approximation.

Within the framework of lattice gauge theory, one can employ either approximate, analytical methods or a direct attack by numerical simulation. Our work exploits both approaches.

The zero-temperature pure gauge theory has no adjustable parameters. This is part of the reason that it is so difficult to approximate. A study of the theory at finite temperature introduces a parameter $T$ that can be varied in a controlled way so as to probe different aspects of the theory. In particular, confinement, which is apparently present at low temperature, is absent at sufficiently high temperature. A study of the theory as the temperature is changed can yield useful information about the features that distinguish these two phases and thus contribute to an understanding of the vacuum state. Two completed works and two active projects deal with the finite-temperature theory.

The first completed work is closely related to the flux tube model for the finite-temperature phase transition. We have been able to correlate certain physical properties of an interacting random walk with the critical exponent for the vanishing string tension at the deconfining phase transition.

The second completed project explores the connection between the finite- temperature quantum gauge field and a classical statistical system at criticality. Universality relates the important properties of the two systems. The comparison of supposedly universal functions is a tough test for these ideas.

Two active projects are concerned with $S U(2)$ gauge fields near the finite-temperature phase transition. The first is an analytical approximation developed to give a physical understanding of the value of the adjoint Wilson line at $T=T_{c}$ that was computed numerically in earlier work. The second is a numerical and analytical study of the critical properties of a flux model that can be used above $T_{c}$

The third proposed work will explore a different direction. The low momentum structure of hadrons is experimentally important but not accessible in ordinary perturbative calculations. In particular, it is not currently possible to calculate the form of the structure functions. Numerical simulations applied to lattice gauge theory can address the issue, but it is quite demanding of computational resources. A particularly interesting topic of impending experimental importance is the power of the small $x$ behavior of the gluon structure function. In a new project, we are engaged in feasibility studies for Monte Carlo calculations that bear on this issue. 
The project above and many others are limited by the practical matter of finite computer resources. Algorithms that can use the existing resources more effectively are welcome. One big problem is the critical slowing down caused by growing correlation lengths. The multigrid approach promises to give substantial relief in that area. Our fourth proposed project will study and develop this new technique.

The following two projects are essentially complete but not yet written-up.

1. J. Kiskis, R. Narayanan, and P. Vranas, "Random Walks and Critical Exponents in Quantum Field Theory".

In the flux tube model of the $\mathrm{SU}(2)$, finite-temperature phase transition, a tube of color electric flux is formed between a static quark and an anti-quark. The deconfining phase transition occurs when the entropy of the fluctuations of the tube overcomes the energy cost of adding to the length of the tube. Early work on this model neglected the interactions of the tube with itself and with background loops of flux. This simple picture of a free random walk of flux correctly predicts that the transition is second order but incorrectly gives gaussian critical exponents. In previous work, we stressed that an improved physical picture which includes both interactions of the tube with itself and with the background loops is needed in order to understand the correct, nontrivial exponents. We showed that the exponent $\nu$ associated with the divergence of the correlation length is the ratio of an exponent related to the energy cost per step and the Hausdorff dimension of the walk.

In this work, we have investigated the problem in more detail in the context of quantum field theories that are related to the gauge theory by universality. On one hand, field theories can be given a random walk representation that provides an interesting physical picture that is complementary to textbook treatments. On the other hand, the calculations of critical exponents in field theory are not very illuminating. We have been able to establish a connection between these two approaches that associates with the exponents some physical intuition. If the random walk had only shortrange, repulsive self interactions, the exponent $\nu$ would be the inverse of the Hausdorff dimension of a self avoiding walk - about 0.59 . For a free walk, the value is 0.5 , but the "correct" answer is 0.63. Including the effects of the background loops lowers the strength of the self interaction and increases the dimension of the walk. However, as the field goes critical the background loops have singular behavior, and the energy per step of the walk is not analytic in the bare mass. We have been able to identify this additional nonanalyticity with the crossover exponent that is discussed for other reasons in the context of the critical behavior of statistical systems. The exponent $\nu$ is then the ratio of the crossover exponent and the dimension of the self-interacting walk. The result is a physical understanding of the exponent $\nu$ and of the crossover exponent.

Now, upon returning to the gauge theory, we can give the numerical values for the Hausdorff dimension of the color flux tube when considered on different length scales. This is a nice improvement in our physical understanding of the deconfinement phase transition.

2. J. Kiskis and S. Staniford-Chen, "Universal Functions of Finite-Size Effects in the SU(2), FiniteTemperature Phase Transition".

The pure glue, $S U(2)$, finite-temperature, deconfining phase transition is second order. In the renormalization group picture, second order transitions are divided into universality classes. The $S U(2)$ transition is in the universality class of the three dimensional Ising model. Transitions in the same class have many common properties. The critical exponents that describe the singular behavior of observables are the same and have been extensively studied. Among high energy physicists, it is not as well known that there are also universal amplitudes and functions. In this project, we have studied the universality of functions associated with finite-size scaling.

Methods developed in the context of critical phenomena are applied to the gauge theory phase transition. Using published ${ }^{[14]}$ Monte Carlo data, we can extract numerical results for some of these universal functions. As a stringent test of universality, they can be compared with Ising model numerical results and with quartic scalar field theory $\epsilon$ expansions. There is qualitative and rough 
quantitative agreement. However, there are some definite quantitative discrepancies. The systematic trends in these suggest that some of the data are not close enough to the critical point.

To the extent that the comparisons are satisfactory, they confirm and deepen our understanding of the gauge theory and its relationship to statistical systems. However, the quantitative missmatches serve to remind use that even our most ambitious numerical simulations suffer from inadequacies. 
Ling-Lie Chau

\section{Nonleptonic Heavy Quark Decays and CP Noninvariance}

Found ways to measure the hairpin diagrams in charmed meson decays (paper 91-3)

The possible presence of hairpin diagrams is analyzed in the model-independent quarkdiagram scheme for two-body decays of charmed mesons. Current experimental data do not require the presence of hairpin diagrams in $D \rightarrow V P(V$ : vector meson, $P$ :pseudoscalar meson), in accordance with the OZI rule. However, there is a possible indication that they are important in the decay of $D \rightarrow P P$. The measurement of $D_{s}^{+} \rightarrow \pi^{+} \eta^{\prime}$ is crucial to test the mechanism of hairpin diagrams.

Studied the charmless nonleptonic decays of $B$ mesons using the quark-diagramscheme and model calculations (paper 91-1. )

Branching ratios of nonleptonic exclusive two-body decays of charged $B$ mesons without final-state charm particles are studied in detail. The technique used for evaluating the nonleptonic decay amplitudes is elucidated. Charmless decay channels, such as $B_{u}^{+} \rightarrow K^{+} \eta^{\prime}$, $\pi^{+} \eta^{\prime}, \rho^{+} \pi^{0}, \rho^{+} \eta, \rho^{+} \eta^{\prime}, B_{d}^{0} \rightarrow K^{+} \pi^{-}, \eta \eta^{\prime}, \eta^{\prime} \eta^{\prime}, K^{*+} \pi^{-}, \rho^{+} \pi^{-}, K^{*+} \rho^{-}, \rho^{+} \rho^{-}$, should be accessible experimentally in the near future. The implications of the quark-diagram scheme are also discussed.

\section{Field Theories for Strong Interactions and Gravity from the Integrable-System. Point of View}

Generated $N$-soliton solutions in two-dimensions (Paper 91-2)

Using the special Riemann-problem technique of Zakharov et al. [Sov. Phys. JETP 47, 1017 (1978)], an explicit $N$-step Bäcklund transformation is derived for a class of $1+$ 1-dimensional nonlinear evolution equations, thus providing an alternative explicit expression of their $\mathrm{N}$-soliton solutions which no longer involves computations of the intermediate solutions, nor leaves any expansion of $N \times N$ determinants (and of their differentiation) to be done.

91-1 Charmless Nonleptonic Rare Decays of B Mesons

(163) Physical Review D43, 2176

Ling-Lie Chau, H.-Y. Cheng, W.K. Sze, H. Yao, and B. Tseng

91-2 An Alternative Explicit Construction of N-Soliton Solutions in Two Dimensions

(164) Journal of Mathematical Physics 32, 1737

Ling-Lie Chau, J.C. Shaw, and H.C. Yen 
91-3 Ways to Measure the Hairpin Diagrams in Charmed Meson Decays

Zeitschrift für Physik C, 53, (1992), 413

Ling-Lie Chau, H.Y. Cheng, and T. Huang

91-4 Overview of $B$ and $K$ Decays

(166) Invited talk and to appear in Proceedings of Conference on

"Rare and Exclusive B and K Decays and Novel Flavor Factories", Santa Monica, California;

Ling-Lie Chau

91-5 Nonleptonic B,D,K Meson Decays and CP Noninvariance

(167) Invited talk and appeared in the Proceedings of the Workshop on Physics and Detectors for KEK

Asymmetric B Factory, Tsukuba, Japan, April 15-18, 1991;

Ling-Lie Chau

91-6 Quantization of the Self-Dual Yang-Mills System and the WZNW Model:

(168) Quadratic and Exchange Algebras, and Modified Yang-Baxter Relations Systems

Invited talk and appeared in the Proceedings of the $\mathrm{XX}^{\text {th }}$ Int'l Conf. on Differential Geometric Methods in Theoretical Physics, June 3-7, 1991, New York;

Ling-Lie Chau and Itaru Yamanaka 
Albert Schwarz

Recent Research Accomplishments

I wrote a series of papers devoted to the investigation of some mathematical problems arising in the theory of non-critical strings. In particular, I described the space of solutions to the equation $[\mathrm{P}, \mathrm{Q}]=$ const. where $\mathrm{P}$ and $\mathrm{Q}$ are ordinary differential operators. (This equation arises in the study of non-critical strings by means of matrix models and therefore it is known as string equation.). I also proved that the generalization of string equation to the supersymmetric case is connected with the superanalogs of KP-hierarchy and studied (in collaboration with M. Bowick and $\mathrm{K}$. Anagnostopoulos) the modification of string equation arising in the theory of unitary matrix models.

I recently started the study of geometry of quantization of constrained system. In the paper that I wrote on this subject I obtained, in particular, very general statement about independence of the choice of gauge condition in the BatalinVilkovisky quantization. I hope that I will be able to find a modification of BatalinVilkovisky quantization that will lead to simplification of calculations and to possibility to give a rigorous justification of many heuristic considerations. I already know some results in this direction within the framework of semi-classical approximation.

1991 Schwarz, Albert. Geometry of fermionic string. Invited lecture at International Congress of Mathematicians, Kyoto, 1990, Springer-Verlag.

1991 Kac, V. and Albert Schwarz. Geometric interpretation of partition function of 2D gravity. Phys. Lett. 257B:329.

1991 Schwarz, A.S. On some mathematical problems of 2D-gravity and $W_{h}$-gravity. Modern Physics Letters A(6)7:611-616. 